\title{
LOCAL INDEX THEORY AND THE RIEMANN-ROCH-GROTHENDIECK THEOREM FOR COMPLEX FLAT VECTOR BUNDLES
}

\author{
MAN-HO HO
}

\begin{abstract}
The purpose of this paper is to give a proof of the real part of the Riemann-Roch-Grothendieck theorem for complex flat vector bundles at the differential form level in the even dimensional fiber case. The proof is, roughly speaking, an application of the local family index theorem for a perturbed twisted spin Dirac operator, a variational formula of the Bismut-Cheeger eta form without the kernel bundle assumption in the even dimensional fiber case, and some properties of the Cheeger-Chern-Simons class of complex flat vector bundle.
\end{abstract}

\section{Contents}

1. Introduction

1.1. Method of proof

1.2. Outline

Acknowledgement

2.1. Chern character form and Chern-Simons form 8

2.2. Cheeger-Chern-Simons class 11

2.3. Local index theory for twisted de Rham operator 14

2.4. Local index theory for twisted spin Dirac operator 18

2.5. The analytic index in differential $K$-theory 23

3. Main results 24

3.1. A variational formula of the Bismut-Cheeger eta form without the kernel bundle assumption and its applications $\quad 24$

3.2. Some properties of Cheeger-Chern-Simons class 33

3.3. The real part of the Riemann-Roch-Grothendieck theorem for complex flat vector bundles

References

2010 Mathematics Subject Classification. Primary 58J20, 19L10, 58J28; Secondary 19L50, $19 \mathrm{~K} 56$.

Key words and phrases. Riemann-Roch-Grothendieck theorem, Cheeger-ChernSimons class, local family index theorem, Bismut-Cheeger eta form. 


\section{INTRODUCTION}

Let $\pi: X \rightarrow B$ be a submersion with closed fibers $Z$ and $F \rightarrow X$ a complex flat vector bundle with flat connection $\nabla^{F}$. The Riemann-RochGrothendieck (RRG) theorem (1.0.1) for complex flat vector bundles is an equality in $H^{\text {odd }}(B ; \mathbb{C} / \mathbb{Q})$ stating that

$$
\left.\operatorname{CCS}\left(H\left(Z,\left.F\right|_{Z}\right), \nabla^{H\left(Z,\left.F\right|_{Z}\right)}\right)\right)=\int_{X / B} e\left(T^{V} X\right) \cup \operatorname{CCS}\left(F, \nabla^{F}\right),
$$

where $\operatorname{CCS}\left(F, \nabla^{F}\right)$ is the Cheeger-Chern-Simons class of $\left(F, \nabla^{F}\right)[27,(1.1)]$ and $H\left(Z,\left.F\right|_{Z}\right) \rightarrow B$ is the cohomology bundle with flat connection $\nabla^{H\left(Z,\left.F\right|_{Z}\right)}$. Both sides of $(1.0 .1)$ are defined in terms of the $\bmod \mathbb{Q}$ reduction of the de Rham class of certain closed odd differential forms.

Bismut-Lott prove the imaginary part of $(1.0 .1)$ at the differential form level [11, Theorem 3.23]. The real part of (1.0.1), referred as the real RRG theorem, is an equality in $H^{\text {odd }}(X ; \mathbb{R} / \mathbb{Q})$ stating that

$$
\operatorname{Re}\left(\operatorname{CCS}\left(H\left(Z,\left.F\right|_{Z}\right), \nabla^{H\left(Z,\left.F\right|_{Z}\right)}\right)\right)=\int_{X / B} e\left(T^{V} X\right) \cup \operatorname{Re}\left(\operatorname{CCS}\left(F, \nabla^{F}\right)\right) .
$$

1.0.2 is first proved by Bismut under the assumption that the fibers are fiberwise orientable [7, Theorem 3.2], and later proved by Ma-Zhang in full generality [27, Theorem 1.1].

The main result of this paper is a $\mathbb{Z}_{2}$-graded version of $(1.0 .2)$ at the differential form level for $\operatorname{dim}(Z)$ even (Theorem 1), i.e. the complex flat vector bundle and its flat connection $\left(F, \nabla^{F}\right)$ in $(1.0 .2)$ are $\mathbb{Z}_{2}$-graded and $F \rightarrow X$ has virtual rank zero. By taking an appropriate $\left(F, \nabla^{F}\right)$ in Theorem 1 we recover a result by Ma-Zhang [27, (3.98)] for $\operatorname{dim}(Z)$ even. By arguing as in [27, p.614], which makes use of a result by Bismut [7, Theorem 3.12], we obtain 1.0 .2 for $\operatorname{dim}(Z)$ even. Along the way we prove a variational formula of the Bismut-Cheeger eta form without the kernel bundle assumption, which could be of independent interest. A brief description of the main results is given in Section 1.1 .

The proof of (1.0.2) by Bismut [7] makes critical use of adiabatic limit computations of the reduced $\eta$-invariant of certain Dirac operators and Cheeger-Simons' geometric index theorem [15, Theorem 9.2]. On the other hand, the proof of 1 1.0.2 by Ma-Zhang [27] uses adiabatic limit computations of the reduced $\eta$-invariant of the sub-signature operator developed by Zhang [30].

Our proof of Theorem 1 makes use of the local family index theorem (local FIT) for a perturbed twisted spin Dirac operator and some properties of the Cheeger-Chern-Simons class, and does not involve the reduced $\eta$-invariant nor adiabatic limit calculations. However, since the result by Bismut [7, Theorem 3.12] we use to derive $(1.0 .2)$ for $\operatorname{dim}(Z)$ even is proved by using the reduced $\eta$-invariant and adiabatic limit calculations, our proof of 1.0 .2 for $\operatorname{dim}(Z)$ even still uses these tools. 
Since for $\operatorname{dim}(Z)$ odd, the right-hand side of $(1.0 .2)$ is zero, the odd dimensional fiber case of the real RRG theorem states that the left-hand side of 11.0 .2 is zero. It is well known that most of the local family index type theorems in the odd dimensional fiber case can be proved by applying a trick due to Bismut-Freed (see the proof of [10. Theorem 2.10] and also [17, §9]) and the corresponding local FIT in the even dimensional fiber case. However, we are unable to give another proof of the odd dimensional fiber case of $(1.0 .2)$ at this moment. The reason seems to be the incompatibility of our techniques and the trick by Bismut-Freed. However, we have an idea to overcome the incompatibility, and the same idea can also be applied to give another proof of the odd dimensional fiber case of the GrothendieckRiemann-Roch theorem in flat $K$-theory (flat GRR theorem) [25] at the differential form level. These questions will be treated in a future paper.

1.1. Method of proof. In this subsection we describe the main results in this paper and outline the method of proof.

The motivation of proving Theorem 1 comes from a fundamental but crucial observation by Ma-Zhang [27, (2.44)] that for a complex flat vector bundle $F \rightarrow X$ with flat connection $\nabla^{F}$ equipped with a Hermitian metric $g^{F}$, where $\nabla^{F}$ is not assumed to be unitary with respect to $g^{F}$, the real part of the Cheeger-Chern-Simons class $\operatorname{CCS}\left(F, \nabla^{F}\right)$ is given by

$$
\operatorname{Re}\left(\operatorname{CCS}\left(F, \nabla^{F}\right)\right)=\left[\frac{1}{k} \operatorname{CS}\left(\nabla_{0}^{k F}, k \nabla^{F, u}\right)\right] \bmod \mathbb{Q} \in H^{\text {odd }}(X ; \mathbb{R} / \mathbb{Q}),
$$

where $k \in \mathbb{N}$ is such that $k F \cong k \mathbb{C}^{\operatorname{rank}(F)}$ as smooth complex vector bundles, $\nabla^{F, u}$ is a unitary connection on $F \rightarrow X$ constructed out of $\nabla^{F}$ and $\nabla_{0}^{k F}$ is a trivial connection on $k F \rightarrow X$. The details will be given in Section 2.3. By considering $\mathcal{F}:=\left(F, g^{F}, \nabla^{F, u}, 0\right)$ as a generator of the flat $K$-group $K_{\mathrm{L}}^{-1}(X)$ [25, Definition 5], (1.1.1) can be written as

$$
\operatorname{Re}\left(\operatorname{CCS}\left(F, \nabla^{F}\right)\right)=-\operatorname{ch}_{\mathbb{R} / \mathbb{Q}}(\mathcal{F}),
$$

where $\operatorname{ch}_{\mathbb{R} / \mathbb{Q}}: K_{\mathrm{L}}^{-1}(X) \rightarrow H^{\text {odd }}(X ; \mathbb{R} / \mathbb{Q})$ is the flat Chern character $[25$, Definition 9]. On the other hand, given a submersion $\pi: X \rightarrow B$ with closed, oriented and $\operatorname{spin}^{c}$ fibers and a $\mathbb{Z}_{2}$-graded generator $\mathcal{E}$ of $K_{\mathrm{L}}^{-1}(X)$, the flat GRR theorem [25, Corollary 4] is an equality in $H^{\text {odd }}(B ; \mathbb{R} / \mathbb{Q})$ stating that

$$
\operatorname{ch}_{\mathbb{R} / \mathbb{Q}}\left(\operatorname{ind}_{\mathrm{L}}^{\mathrm{a}}(\mathcal{E})\right)=\int_{X / B} \operatorname{Todd}(X / B) \cup \operatorname{ch}_{\mathbb{R} / \mathbb{Q}}(\mathcal{E}),
$$

where $\operatorname{ind}_{\mathrm{L}}^{\mathrm{a}}: K_{\mathrm{L}}^{-1}(X) \rightarrow K_{\mathrm{L}}^{-1}(B)$ is the analytic index in flat $K$-theory [25, Definition 14]. By comparing (1.0.2) and (1.1.3) using (1.1.2) we wonder if the real RRG theorem can be proved in the same way as the flat GRR theorem given in [20].

Since the main idea of the proof of Theorem 1 is similar to that of the flat GRR theorem given in [20], we briefly recall it here. Given the setup of 
the flat GRR theorem described above, consider the associated submersion $\widetilde{\pi}: \widetilde{X} \rightarrow \widetilde{B}$, where $I=[0,1], \widetilde{X}=X \times I$ (similarly for $\widetilde{B}$ ) and $\widetilde{\pi}:=\pi \times$ id. For any manifold $X$, define a map $i_{X, k}: X \rightarrow \widetilde{X}$ by $i_{X, k}(x)=(x, k)$. Given a $\mathbb{Z}_{2}$-graded generator $\left(E, g^{E}, \nabla^{E}, \phi\right)$ of $K_{\mathrm{L}}^{-1}(X)$, we construct a complex vector bundle $\mathscr{E} \rightarrow \widetilde{X}$ with a Hermitian metric $g^{\mathscr{E}}$ and a unitary connection $\nabla^{\mathscr{E}}$ such that $i_{X, 1}^{*} \nabla^{\mathscr{E}}=m \nabla^{E^{+}}$and $i_{X, 0}^{*} \nabla^{\mathscr{E}}=m \nabla^{E^{-}}$for some $m \in \mathbb{N}$ satisfying $m E^{+} \cong m E^{-}$. Assume the family of kernels $\operatorname{ker}\left(\mathrm{D}_{\widetilde{b}}^{S^{c} \otimes \mathscr{E}}\right)$ of the twisted $\operatorname{spin}^{c}$ Dirac operator $\mathrm{D}^{S^{c} \otimes \mathscr{E}}$ parameterized by $\widetilde{B}$ form a $\left(\mathbb{Z}_{2}\right.$-graded $)$ complex vector bundle. The local FIT for $\mathrm{D}^{S^{c} \otimes \mathscr{E}}$ is given by

$$
d \widetilde{\eta}=\int_{\widetilde{X} / \widetilde{B}} \operatorname{Todd}\left(\nabla^{S^{c}\left(T^{V} \widetilde{X}\right)}\right) \wedge \operatorname{ch}\left(\nabla^{\mathscr{E}}\right)-\operatorname{ch}\left(\nabla^{\operatorname{ker}\left(\mathrm{D}^{S^{c} \otimes \mathscr{E}}\right)}\right),
$$

where $\widetilde{\eta}$ is the Bismut-Cheeger eta form associated to $\mathrm{D}^{S^{c} \otimes \mathscr{E}}$. By integrating 1.1.4 along the fibers of the trivial fibration $\widetilde{B} \rightarrow B$ we obtain an equality of closed odd differential forms refining (1.1.3).

At a first glance, one may suspect that the above strategy can be directly applied to prove Theorem 1 if the $\operatorname{spin}^{c}$ Dirac operator is replaced by the de Rham operator. However, the above strategy causes two problems in the current situation.

For the first problem, recall that the local FIT for the twisted de Rham operator $\mathrm{D}^{\widetilde{Z}, \mathrm{dR}}$ for $\widetilde{\pi}: \widetilde{X} \rightarrow \widetilde{B}[7, \S 3(\mathrm{c})]$ states that

$$
d \widetilde{\eta}^{\mathrm{dR}}=\int_{\widetilde{X} / \widetilde{B}} e\left(\nabla^{T^{V} \widetilde{X}}\right) \wedge \operatorname{ch}\left(\nabla^{\mathscr{F}, u}\right)-\operatorname{ch}\left(\nabla^{H(\widetilde{Z}, \mathscr{F} \mid \widetilde{Z}), u}\right),
$$

where $\mathscr{F} \rightarrow \tilde{X}$ is a complex flat vector bundle with flat connection $\nabla^{\mathscr{F}}$, and $\widetilde{\eta}^{\mathrm{dR}}$ is the Bismut-Cheeger eta form associated to $\mathrm{D}^{\widetilde{Z}}, \mathrm{dR}$. Since $\operatorname{ch}\left(\nabla^{\mathscr{F}, u}\right)=$ $\operatorname{rank}(\mathscr{F})$ and

$$
\left.\operatorname{ch}\left(\left.\nabla^{H(\widetilde{Z}, \mathscr{F}}\right|_{\widetilde{Z}}\right), u\right)=\operatorname{rank}\left(H\left(\widetilde{Z},\left.\mathscr{F}\right|_{\widetilde{Z}}\right)\right)=\operatorname{rank}(\mathscr{F}) \chi(\widetilde{Z}),
$$

the right-hand side of 1.1 .5 is zero, and therefore $\widetilde{\eta}^{\mathrm{dR}}$ is closed. A stronger result by Bismut [7, Theorem 3.7] states that $\widetilde{\eta}^{\mathrm{dR}}=0$. Thus integrating 1.1.5 along the fibers of $\widetilde{B} \rightarrow B$ gives $0=0$ instead of an equality of closed odd differential forms refining (1.0.2).

The second problem is the assumption of the existence of the kernel bundle $\operatorname{ker}\left(\mathrm{D}^{S^{c} \otimes \mathscr{E}}\right) \rightarrow \widetilde{B}$ in our proof of the flat GRR theorem. It is well known that if the kernel bundle does not exist, then one can perturb the Dirac operator so that the resulting family of kernels form a vector bundle. Thus the statement can usually be reduced to the kernel bundle case. This reduction process can be applied to the flat GRR theorem, but not to the real RRG theorem. For the flat GRR theorem, if the kernel bundle exists then the bundle part of the analytic index in flat $K$-theory is defined to be the kernel bundle; otherwise the bundle part is defined to be any fixed choice of a finite rank subbundle $L \rightarrow B$ in the approach by Miščenko-Fomenko, which is 
outlined below. Thus the flat GRR theorem at the differential form level can be proved by reducing it to the kernel bundle case. For Theorem 1, however, there is a specific "target" regardless of the existence of the kernel bundle of a suitable Dirac operator, namely, the cohomology bundle $H\left(Z,\left.F\right|_{Z}\right) \rightarrow$ $B$. If we prove Theorem 1 under the kernel bundle assumption, whose existence is actually unknown in reality, we would have to pull back, for example, a unitary connection on the possibly non-existing kernel bundle to $H\left(Z,\left.F\right|_{Z}\right) \rightarrow B$ (see (1.1.7)). This argument is certainly incorrect. We would like to thank the referee for pointing this out.

Before we outline the solutions to these two problems, let us briefly recall that there are (at least) two approaches to deal with the non-existence of the kernel bundle.

- One approach is given by Atiyah-Singer 3] (see also [4, §9.5]). The idea is to find a trivial bundle $\mathbb{C}^{N} \rightarrow B$ and a linear map $s: \mathbb{C}^{N} \rightarrow$ $\left(\pi_{*} E\right)^{-}$in order to perturb the Dirac operator $\mathrm{D}$ by a smoothing operator $R_{s}$ induced by $s$, so that $\left(\mathrm{D}+R_{s}\right)_{+}:\left(\pi_{*} E\right)^{+} \oplus \mathbb{C}^{N} \rightarrow$ $\left(\pi_{*} E\right)^{-}$is surjective, and therefore $\operatorname{ker}\left(\left(\mathrm{D}+R_{s}\right)_{-}\right)=0$. In this case the $K$-theoretic analytic index of $[E] \in K(X)$ is defined to be $\left[\operatorname{ker}\left(\mathrm{D}+R_{s}\right)\right]-\left[\mathbb{C}^{N}\right]$.

- Another approach is given by Miščenko-Fomenko [28, Lemma 2.2]. The idea is to find a $\mathbb{Z}_{2}$-graded finite rank subbundle $L \rightarrow B$ of $\pi_{*} E \rightarrow B$ and a $\mathbb{Z}_{2}$-graded complementary subbundle $K \rightarrow B$ such that $\mathrm{D}_{+}$is block diagonal with respect to the decomposition $\left(\pi_{*} E\right)^{ \pm}=K^{ \pm} \oplus L^{ \pm}$and it restricts to an isomorphism $K^{+} \rightarrow K^{-}$. In this case the $K$-theoretic analytic index of $[E] \in K(X)$ is defined to be $\left[L^{+}\right]-\left[L^{-}\right]$.

The idea of solving the above problems is, roughly speaking, to consider the following perturbed twisted spin Dirac operator

$$
\mathrm{D}^{S \widehat{\otimes}\left(S^{*} \otimes \mathscr{F}\right)}+\tilde{V} \in \Gamma\left(\tilde{X}, \operatorname{End}^{-}\left(S\left(T^{V} \tilde{X}\right) \widehat{\otimes}\left(S\left(T^{V} \tilde{X}\right)^{*} \otimes \mathscr{F}\right)\right)\right),
$$

where the details will be given in Section 2.3 . The unitary connection $\nabla^{\mathscr{F}}, u$ on $\mathscr{F} \rightarrow \widetilde{X}$ defining $\mathrm{D}^{S \widehat{\otimes} S^{*} \otimes \mathscr{F}}$ is chosen in the way that its curvature satisfies a certain condition (namely, 2.2 .4$)$ ) only on $\left.\left(S\left(T^{V} \widetilde{X}\right)^{*} \otimes \mathscr{F}\right)\right|_{\partial \widetilde{X}} \rightarrow \partial \widetilde{X}$. The reason of perturbing and twisting the spin Dirac operator as in 1.1 .6 is due to

$$
S\left(T^{V} X\right) \widehat{\otimes} S\left(T^{V} X\right)^{*} \cong \Lambda\left(T^{V} X\right)^{*} \otimes \mathbb{C}
$$

as $\mathbb{Z}_{2}$-graded complex vector bundles and the following result by BismutZhang [12, Proposition 4.12]:

$$
\mathrm{D}^{\Lambda \otimes F}+V=\mathrm{D}^{Z, \mathrm{dR}}
$$

where the curvature of the unitary connection $\nabla^{F, u}$ defining $\mathrm{D}^{\Lambda \otimes F}$ satisfies (2.2.4). If we assumed the existence of the kernel bundle of the Dirac operator (1.1.6), then one could prove that

$$
\operatorname{ker}\left(\mathrm{D}^{S \widehat{\otimes}\left(S^{*} \otimes \mathscr{F}\right)}+\widetilde{V}\right) \cong \operatorname{ker}\left(\mathrm{D}^{\Lambda \otimes \mathscr{F}}+\widetilde{V}\right)
$$


as $\mathbb{Z}_{2}$-graded complex vector bundles. This would imply

$$
i_{B, k}^{*} \operatorname{ker}\left(\mathrm{D}^{S \widehat{\otimes}\left(S^{*} \otimes \mathscr{F}\right)}+\widetilde{V}\right) \cong i_{B, k}^{*} \operatorname{ker}\left(\mathrm{D}^{\Lambda \otimes \mathscr{F}}+\widetilde{V}\right) \cong i_{B, k}^{*} \operatorname{ker}\left(\mathrm{D}^{\widetilde{Z}, \mathrm{dR}}\right)
$$

as $\mathbb{Z}_{2}$-graded complex vector bundles over $B$ for $k \in\{0,1\}$. However, our assumption is not verified in general, so we need to adopt one of the two aforementioned approaches.

We choose the approach given by Miščenko-Fomenko, and the corresponding results of the local FIT in this approach is given by Freed-Lott [17, $\S 7]$. More precisely, we first establish a variational formula of the BismutCheeger eta form without the kernel bundle assumption (Proposition 1). As a byproduct we give another proofs of the facts that

- the analytic index in differential $K$-theory defined without the kernel bundle assumption does not depend on the choice of the finite rank subbundle $L \rightarrow B$ (Corollary 1),

- if the kernel bundle exists then the two definitions of the analytic index in differential $K$-theory, under and without the kernel bundle assumption, coincide as elements in the differential $K$-group (Corollary 2).

These results are first proved by Freed-Lott 17, (3) and (4) of Corollary 7.36] as a consequence of the FIT in differential $K$-theory [17, Theorem 7.35]. Our proofs of these results are an application of Proposition 1, and do not make use of the FIT in differential $K$-theory. Note that these results are stated in terms of spin fibers in this paper, as opposed to [17], which are stated in terms of $\operatorname{spin}^{c}$ fibers. By a minor modification all the results in Section 3.1 can be extended to spin $^{c}$ fibers case.

The idea of proving Theorem 1 follows closely to the proof of Proposition 1. In more detail, given a $\mathbb{Z}_{2}$-graded complex flat vector bundle $F \rightarrow X$ of virtual rank zero with $\mathbb{Z}_{2}$-graded flat connection $\nabla^{F}$, we take $k \in \mathbb{N}$ large enough so that there exist smooth bundle isomorphisms $j: k F^{+} \rightarrow$ $k F^{-}$and $h_{ \pm}^{\bullet}: k H^{\bullet}\left(Z,\left.F^{ \pm}\right|_{Z}\right) \cong \mathbb{C}^{k n_{ \pm}^{\bullet}}$ for $\bullet \in$ even, odd $\}$, where $n_{ \pm}^{\bullet}=$ $\operatorname{rank}\left(H^{\bullet}\left(Z,\left.F^{ \pm}\right|_{Z}\right)\right)$. Then by putting a $\mathbb{Z}_{2}$-graded Hermitian metric $g^{\bar{F}}$ on $F \rightarrow X$, there exists a smooth isometric isomorphism $\widetilde{j}: k F^{+} \rightarrow k F^{-}$ associated to $\left(k, j, g^{F}\right)$, i.e. $k g^{+}=\widetilde{j}^{*} k g^{-}$. Note that $k \nabla^{+}$and $\widetilde{j}^{*} k \nabla^{-}$are two flat connections on $k F^{+} \rightarrow X$. By joining $k \nabla^{+}$and $\widetilde{j}^{*} k \nabla^{-}$by a smooth path of connections, one can construct a Hermitian bundle $\mathscr{F} \rightarrow \widetilde{X}$ with a unitary connection $\nabla^{\mathscr{F}}, u$ which pulls back to two unitary connections on $k F^{+} \rightarrow X$ associated to $k \nabla^{+}$and $\widetilde{j}^{*} k \nabla^{-}$, respectively. By considering the perturbed twisted spin Dirac operator (1.1.6) without assuming the existence of its kernel bundle, one obtains an analog of local FIT that is suitable for our purpose. We then integrate the so obtained equality of closed differential forms along the fibers of the trivial fibration $\widetilde{B} \rightarrow B$. Since the kernel bundle exists over $\partial \widetilde{B}$, the idea is to "replace" the pullback of the $\mathbb{Z}_{2}$-graded finite rank subbundle $\mathcal{L} \rightarrow \widetilde{B}$ to $\partial \widetilde{B}$ by the kernel bundle. At this step we cannot directly apply Corollary 2, but it provides a guidance on what and how 
the geometric data should be replaced. The reason that Corollary 2 is not directly applicable here is due to the perturbed twisted spin Dirac operator (1.1.6). The definition of the analytic index in differential $K$-theory without the kernel bundle assumption does not allow us to perturb $\mathrm{D}^{S \widehat{\otimes}\left(S^{*} \otimes \mathscr{F}\right)}$ by $\widetilde{V}$. Thus we are forced to mimic the proof of Proposition 1 when proving Theorem 1. During the process of "replacing" the kernel bundle over $\partial \widetilde{B}$ the isomorphism

$$
\widetilde{j}:\left(k F^{+}, \tilde{j}^{*} k \nabla^{-}\right) \rightarrow\left(k F^{-}, k \nabla^{-}\right)
$$

of complex flat vector bundles plays an important role. The proof of Theorem 1 is completed by mimicking the proof of Proposition 1 adapted to the current situation. By taking an appropriate $\mathbb{Z}_{2}$-graded complex flat vector bundle with $\mathbb{Z}_{2}$-graded flat connection in Theorem 11, and using the properties of the Cheeger-Chern-Simons class established in Section 3.2 and a result by Bismut [7, Theorem 3.12], we deduce (1.0.2 for $\operatorname{dim}(Z)$ even.

We would like to emphasize that the use of differential $K$-theory in this paper is not absolutely necessary (we could state Corollary 1 and Corollary 2 without it). However, differential $K$-theory is a very convenient tool to keep track of the changes of the geometric objects in local index theory when the defining data are deformed, and it effectively shortens the presentation.

1.2. Outline. The paper is organized as follows. In Section 2 we review the background material, including some aspects of the Chern character form and the Chern-Simons form, the Cheeger-Chern-Simons class of complex flat vector bundles, the setup and the statement of the local FIT for twisted de Rham operator, and the local FIT for twisted spin Dirac operator under and without the kernel bundle assumption. In Section 3 we prove the main results in this paper. In Section 3.1 we prove a variational formula of the Bismut-Cheeger eta form without the kernel bundle assumption in the even dimensional fiber case. Then we show the independence of the choice of the $\mathbb{Z}_{2}$-graded finite rank subbundle in the definition of the analytic index in differential $K$-theory without the kernel bundle assumption, and we prove that if the kernel bundle exists then the two definitions of the analytic index in differential $K$-theory coincide. Section 3.2 is devoted to some basic properties of the Cheeger-Chern-Simons class that will be used in Section 3.3. In Section 3.3 we prove the main result of the paper. First of all we prove a $\mathbb{Z}_{2}$-graded version of 1.0 .2$)$ for $\operatorname{dim}(Z)$ even at the differential form level. Then we deduce 1.0.2 for $\operatorname{dim}(Z)$ even.

\section{ACKNOWLEDGEMENT}

We would like to thank Steve Rosenberg for many helpful advice and his constant encouragement, Sebastian Goette for generously answering many (awkward) questions in MathOverflow, and Bo Liu for a discussion on a variational formula of the Bismut-Cheeger eta form. Last but not least we would like to thank the referee(s) for his/her patience, careful reading and 
helpful comments, correcting the mistakes and improving the presentation of the paper significantly.

\section{BACKGROUND MATERIAL}

In this paper $X$ and $B$ are closed manifolds and $I$ is the closed interval $[0,1]$. Given a manifold $X$, define $\widetilde{X}=X \times I$. Given $t \in[0,1]$, define a map $i_{X, t}: X \rightarrow \widetilde{X}$ by $i_{X, t}(x)=(x, t)$. Denote by $p_{X}: \widetilde{X} \rightarrow X$ the standard projection map. For $k \geq 0$, denote by $\Omega_{\mathbb{Q}}^{k}(X ; \mathbb{C})$ the set of all complex-valued closed $k$-forms on $X$ with periods in $\mathbb{Q}$, and write $\Omega_{\mathbb{Q}}^{k}(X)$ for $\Omega_{\mathbb{Q}}^{k}(X ; \mathbb{R})$.

Let $E \rightarrow X$ and $F \rightarrow X$ be complex vector bundles, where $E \rightarrow X$ is $\mathbb{Z}_{2^{-}}$ graded. Denote by $E^{\mathrm{op}} \rightarrow X$ the $\mathbb{Z}_{2}$-graded complex vector bundle whose $\mathbb{Z}_{2}$-grading is the opposite of $E \rightarrow X$, i.e. $\left(E^{\mathrm{op}}\right)^{+}=E^{-}$and $\left(E^{\mathrm{op}}\right)^{-}=E^{+}$. We will also use the notation op for other $\mathbb{Z}_{2}$-graded objects. Denote by $E \otimes F \rightarrow X$ the $\mathbb{Z}_{2}$-graded tensor product if $F \rightarrow X$ is ungraded; and by $E \widehat{\otimes} F \rightarrow X$ the $\mathbb{Z}_{2}$-graded tensor product if $F \rightarrow X$ is $\mathbb{Z}_{2}$-graded.

2.1. Chern character form and Chern-Simons form. In this subsection we recall the definitions of the Chern character form and the ChernSimons form, and also fix the sign convention.

Let $E \rightarrow X$ be a complex vector bundle with a connection $\nabla^{E}$. The Chern character form of $\nabla^{E}$ is defined by

$$
\operatorname{ch}\left(\nabla^{E}\right)=\operatorname{tr}\left(e^{-\frac{1}{2 \pi i}\left(\nabla^{E}\right)^{2}}\right) \in \Omega_{\mathbb{Q}}^{\text {even }}(X ; \mathbb{C}) .
$$

There is a "canonical" transgression form $\operatorname{CS}\left(\nabla_{0}^{E}, \nabla_{1}^{E}\right) \in \frac{\Omega^{\text {odd }}(X ; \mathbb{C})}{\operatorname{Im}(d)}$ between the Chern character forms of two connections in the sense that

$$
d \operatorname{CS}\left(\nabla_{0}^{E}, \nabla_{1}^{E}\right)=\operatorname{ch}\left(\nabla_{1}^{E}\right)-\operatorname{ch}\left(\nabla_{0}^{E}\right) .
$$

One of the definitions of $\operatorname{CS}\left(\nabla_{1}^{E}, \nabla_{0}^{E}\right)$ is given as follows. In the following $k \in\{0,1\}$ is fixed. Let $\mathscr{E} \rightarrow \widetilde{X}$ be a complex vector bundle with a connection $\nabla^{\mathscr{E}}$. Since $p_{X} \circ i_{X, k}=\operatorname{id}_{X}$ and $i_{X, k} \circ p_{X} \sim \operatorname{id}_{\tilde{X}}$, it follows that $\mathscr{E} \cong p_{X}^{*}\left(i_{X, k}^{*} \mathscr{E}\right)$. Thus

$$
E_{0}:=i_{X, 0}^{*} \mathscr{E} \cong i_{X, 0}^{*} p_{X}^{*}\left(i_{X, 0}^{*} \mathscr{E}\right) \cong i_{X, 1}^{*} p_{X}^{*}\left(i_{X, 0}^{*} \mathscr{E}\right) \cong i_{X, 1}^{*} \mathscr{E}=: E_{1} .
$$

Write $E=E_{0} \cong E_{1}$. For a fixed $k \in\{0,1\}$, define a connection $\nabla_{k}^{E}$ on $E \rightarrow X$ by

$$
\nabla_{k}^{E}:=i_{X, k}^{*} \nabla^{\mathscr{E}} \text {. }
$$

The Chern-Simons form $\operatorname{CS}\left(\nabla_{0}^{E}, \nabla_{1}^{E}\right)$ is defined to be

$$
\operatorname{CS}\left(\nabla_{0}^{E}, \nabla_{1}^{E}\right)=-\int_{\widetilde{X} / X} \operatorname{ch}\left(\nabla^{\mathscr{E}}\right) \bmod \operatorname{Im}(d),
$$

where $\tilde{X} / X$ denotes the fiber of the fiber bundle $\widetilde{X} \rightarrow X$, and $\int_{\widetilde{X} / X}$ denotes the integration along the fiber. 
We will need some facts about integration along the fibers. Let $\pi: M \rightarrow B$ be a smooth fiber bundle with compact fibers. By [13, Chapter 1], we have

$$
\int_{M / B} \pi^{*} \alpha \wedge \beta=\alpha \wedge\left(\int_{M / B} \beta\right)
$$

for all $\alpha \in \Omega(B)$ and $\beta \in \Omega(M)$. If $M$ is a manifold with boundary and the fiber bundle $\pi: M \rightarrow B$, whose fibers are compact and of dimension $n$, satisfies certain orientability assumptions, then Stokes' theorem for integration along the fibers (see, for example, [14, (1.52)] and [19, Problem 4 (p.311)]) is given by

$$
(-1)^{k-n+1} \int_{\partial M / B} i^{*} \omega=\int_{M / B} d^{M} \omega-d^{B} \int_{M / B} \omega
$$

where $i: \partial M \rightarrow M$ is the inclusion map and $\omega \in \Omega^{k}(M)$.

By considering the fiber bundle $\widetilde{X} \rightarrow X$ and taking $\omega=\operatorname{ch}\left(\nabla^{\mathscr{E}}\right)$ in 2.1.4), we have

$$
\begin{aligned}
d \operatorname{CS}\left(\nabla_{0}^{E}, \nabla_{1}^{E}\right) & =-d \int_{\tilde{X} / X} \operatorname{ch}\left(\nabla^{\mathscr{E}}\right)=-\int_{\tilde{X} / X} d \operatorname{ch}\left(\nabla^{\mathscr{E}}\right)+\int_{\partial \widetilde{X} / X} i^{*} \operatorname{ch}\left(\nabla^{\mathscr{E}}\right) \\
& =\operatorname{ch}\left(\nabla_{1}^{E}\right)-\operatorname{ch}\left(\nabla_{0}^{E}\right) .
\end{aligned}
$$

Thus the Chern-Simons form defined by $(2.1 .3)$ satisfies 2.1 .1$)$.

Given two connections $\nabla_{1}^{E}$ and $\nabla_{0}^{E}$ on $E \rightarrow X$, one can take $\mathscr{E}=p_{X}^{*} E$ in above and define

$$
\nabla^{\mathscr{E}}=\nabla_{t}^{E}+d t \wedge \frac{\partial}{\partial t}
$$

where $\nabla_{t}^{E}$ is a smooth curve of connections joining $\nabla_{0}^{E}$ and $\nabla_{1}^{E}$. Note that $\operatorname{CS}\left(\nabla_{0}^{E}, \nabla_{1}^{E}\right)$ is independent of the choice of $\nabla_{t}^{E}$ (see, for example, [29, Proposition 1.1] and [26, Theorem B.5.4]). In the case that $E \rightarrow X$ is equipped with Hermitian metrics $g_{0}^{E}$ and $g_{1}^{E}$, and $\nabla_{k}^{E}$ is unitary with respect to $g_{k}^{E}$ for $k \in\{0,1\}$, one defines $\nabla^{\mathscr{E}}$ in 2.1 .3 as in [26, p.373]. We recall its construction here. Denote by $\bar{E}^{*} \rightarrow X$ the antidual bundle of $E \rightarrow X$, and by $f_{k}: E \rightarrow \bar{E}^{*}$ the canonical smooth bundle isomorphism associated to $g_{k}^{E}$. Then $f_{0}^{-1} \circ f_{1} \in \operatorname{Aut}(E)$ is positive and self-adjoint with respect to $g_{0}^{E}$ on each fiber. Denote by $f$ its unique positive self-adjoint square root. By the proof of [22, Theorem 8.8 of Chapter 1] we have $g_{1}^{E}=f^{*} g_{0}^{E}$, and therefore $\left(f^{-1}\right)^{*} \nabla_{1}^{E}$ is unitary with respect to $g_{0}^{E}$. Thus the smooth path of connections on $E \rightarrow X$ defined by

$$
\nabla_{t}^{E}=(1-t) \nabla_{0}^{E}+t\left(f^{-1}\right)^{*} \nabla_{1}^{E}
$$

is unitary with respect to $g_{0}^{E}$. Let $f_{t}=(1-t) \mathrm{id}_{E}+t f$. Since $g_{t}^{E}=f_{t}^{*} g_{0}^{E}$ is a smooth path of Hermitian metrics on $E \rightarrow X$ joining $g_{0}^{E}$ and $g_{1}^{E}$, it follows that $f_{t}^{*} \nabla_{t}^{E}$ is a smooth path of unitary connections on $E \rightarrow X$ with respect to $g_{t}^{E}$ for each $t \in[0,1]$ joining $\nabla_{0}^{E}$ and $\nabla_{1}^{E}$. Define a Hermitian metric $g^{\mathscr{E}}$ 
on $\mathscr{E} \rightarrow \widetilde{X}$ by $g^{\mathscr{E}}=p_{X}^{*} g_{t}^{E}$. Then the connection on $\mathscr{E} \rightarrow \widetilde{X}$ defined by

$$
\nabla^{\mathscr{E}}=f_{t}^{*} \nabla_{t}^{E}+d t \wedge\left(\frac{\partial}{\partial t}+\frac{1}{2}\left(g_{t}^{E}\right)^{-1} \frac{\partial}{\partial t} g_{t}^{E}\right)
$$

is unitary with respect to $g^{\mathscr{E}}$.

Another equivalent definition of the Chern-Simons form is given by

$$
\operatorname{CS}\left(\nabla_{1}^{E}, \nabla_{0}^{E}\right)=\int_{0}^{1} \operatorname{tr}\left(\frac{d \nabla_{t}^{E}}{d t} e^{-\frac{1}{2 \pi i}\left(\nabla_{t}^{E}\right)^{2}}\right) d t
$$

The choices of 0 and 1 are immaterial. If $t<T$ are two fixed positive real numbers, then one can replace 0 by $t$ and 1 by $T$ in above.

It follows from (2.1.3) that the Chern-Simons form satisfies the following properties:

$$
\begin{aligned}
\operatorname{CS}\left(\nabla_{1}^{E}, \nabla_{0}^{E}\right) & =-\operatorname{CS}\left(\nabla_{0}^{E}, \nabla_{1}^{E}\right) \\
\operatorname{CS}\left(\nabla_{1}^{E}, \nabla_{0}^{E}\right) & =\operatorname{CS}\left(\nabla_{1}^{E}, \nabla_{2}^{E}\right)+\operatorname{CS}\left(\nabla_{2}^{E}, \nabla_{0}^{E}\right) \\
\operatorname{CS}\left(\nabla_{1}^{E} \oplus \nabla_{1}^{F}, \nabla_{0}^{E} \oplus \nabla_{0}^{F}\right) & =\operatorname{CS}\left(\nabla_{1}^{E}, \nabla_{0}^{E}\right)+\operatorname{CS}\left(\nabla_{1}^{F}, \nabla_{0}^{F}\right) .
\end{aligned}
$$

Let $E \rightarrow X$ be a $\mathbb{Z}_{2}$-graded complex vector bundle with a superconnection $\mathbb{A}$. The Chern character form of $\mathbb{A}$ is defined by

$$
\operatorname{ch}(\mathbb{A})=\operatorname{str}\left(e^{-\frac{1}{2 \pi i} \mathbb{A}^{2}}\right) \in \Omega_{\mathbb{Q}}^{\text {even }}(X ; \mathbb{C}),
$$

where str $: \Gamma\left(X, \Lambda\left(T^{*} X\right) \otimes \mathbb{C} \otimes \operatorname{End}(X)\right) \rightarrow \Omega(X ; \mathbb{C})$ is the extended supertrace [4, Section 1.5]. If $\mathbb{A}_{0}$ and $\mathbb{A}_{1}$ are two superconnections on $E \rightarrow X$, one can define the Chern-Simons form $\operatorname{CS}\left(\mathbb{A}_{0}, \mathbb{A}_{1}\right) \in \frac{\Omega^{\text {odd }}(X ; \mathbb{C})}{\operatorname{Im}(d)}$ in a similar way as 2.1.3) or equivalently 2.1.7). Chern-Simons form of superconnections shares similar properties to Chern-Simons form of ordinary connections, namely,

$$
\begin{aligned}
\operatorname{CS}\left(\mathbb{A}_{0}, \mathbb{A}_{1}\right) & =-\operatorname{CS}\left(\mathbb{A}_{1}, \mathbb{A}_{0}\right) \\
\operatorname{CS}\left(\mathbb{A}_{0}, \mathbb{A}_{1}\right) & =\operatorname{CS}\left(\mathbb{A}_{0}, \mathbb{A}_{2}\right)+\operatorname{CS}\left(\mathbb{A}_{2}, \mathbb{A}_{1}\right), \\
\operatorname{CS}\left(\mathbb{A}_{0}, \mathbb{A}_{1}\right)+\operatorname{CS}\left(\mathbb{B}_{0}, \mathbb{B}_{1}\right) & =\operatorname{CS}\left(\mathbb{A}_{0} \oplus \mathbb{B}_{0}, \mathbb{A}_{1} \oplus \mathbb{B}_{1}\right) .
\end{aligned}
$$

If the superconnections $\mathbb{A}_{0}$ and $\mathbb{A}_{1}$ are $\mathbb{Z}_{2}$-graded connections $\nabla_{0}=\nabla_{0}^{+} \oplus \nabla_{0}^{-}$ and $\nabla_{1}=\nabla_{1}^{+} \oplus \nabla_{1}^{-}$, then one can easily show that

$$
\operatorname{CS}\left(\nabla_{1}, \nabla_{0}\right)=\operatorname{CS}\left(\nabla_{1}^{+}, \nabla_{0}^{+}\right)-\operatorname{CS}\left(\nabla_{1}^{-}, \nabla_{0}^{-}\right) .
$$

2.1.14 holds for $\mathbb{Z}_{2}$-graded unitary connections as well.

Now put a $\mathbb{Z}_{2}$-graded Hermitian metric $g^{E}$ and a $\mathbb{Z}_{2}$-graded unitary connection $\nabla^{E}$ on $E \rightarrow X$. Let $s_{E} \in \Gamma\left(X\right.$, End $\left.^{-}(E)\right)$ be an odd self-adjoint section. The quadruple $\left(E, g^{E}, \nabla^{E}, s_{E}\right)$ is said to split [6, Definition 2.9] if

(1) $E=\operatorname{ker}\left(s_{E}\right) \oplus \operatorname{Im}\left(s_{E}\right)$,

(2) $\nabla^{E}$ preserves $\operatorname{ker}\left(s_{E}\right) \rightarrow X$ and $\operatorname{Im}\left(s_{E}\right) \rightarrow X$, and

(3) $s_{E}$ is a unitary odd section of $\operatorname{End}\left(\operatorname{Im}\left(s_{E}\right)\right) \rightarrow X$ preserving $\nabla^{\operatorname{Im}\left(s_{E}\right)}$. 
Example 1. An example of split quadruple $\left(W, g^{W}, \nabla^{W}, s_{W}\right)$ over $B$ is given by

$$
W^{+}=W^{-}, \quad g^{W^{+}}=g^{W^{-}}, \quad \nabla^{W^{+}}=\nabla^{W^{-}}, \quad s_{W}=\left(\begin{array}{cc}
0 & \mathrm{id} \\
\mathrm{id} & 0
\end{array}\right) .
$$

In this case $\operatorname{ker}\left(s_{W}\right) \rightarrow B$ is the zero $\mathbb{Z}_{2}$-graded complex vector bundle, so the other conditions of $\left(W, g^{W}, \nabla^{W}, s_{W}\right)$ being split are immediately satisfied.

Example 1 will play an important role in proving Proposition 1 .

Let $E \rightarrow X$ be a real vector bundle with a Euclidean metric $g^{E}$ and a Euclidean connection $\nabla^{E}$. The $\widehat{A}$-genus form of $\nabla^{E}$ is defined to be

$$
\widehat{A}\left(\nabla^{E}\right)=\sqrt{\operatorname{det}\left(\frac{-\frac{1}{4 \pi i} R^{E}}{\sinh \left(-\frac{1}{4 \pi i} R^{E}\right)}\right)} \in \Omega_{\mathbb{Q}}^{4 \bullet}(X),
$$

where $R^{E}$ is the curvature of $\nabla^{E}$. If $\nabla_{1}^{E}$ and $\nabla_{0}^{E}$ are two Euclidean connections on $E \rightarrow X$, one can define a transgression form $\widetilde{\widehat{A}}\left(\nabla_{1}^{E}, \nabla_{0}^{E}\right) \in$ $\frac{\Omega^{4 \bullet-1}(X)}{\operatorname{Im}(d)}$ between $\widehat{A}\left(\nabla_{0}^{E}\right)$ and $\widehat{A}\left(\nabla_{1}^{E}\right)$ by

$$
\widetilde{\widehat{A}}\left(\nabla_{0}^{E}, \nabla_{1}^{E}\right)=-\int_{\widetilde{X} / X} \widehat{A}\left(\nabla^{\mathscr{E}}\right),
$$

where $\nabla^{\mathscr{E}}$ is the connection on $\mathscr{E} \rightarrow \widetilde{X}$ defined in a way similar to 2.1.6. Similarly we have

$$
d \widetilde{\widehat{A}}\left(\nabla_{0}^{E}, \nabla_{1}^{E}\right)=\widehat{A}\left(\nabla_{1}^{E}\right)-\widehat{A}\left(\nabla_{0}^{E}\right) .
$$

2.2. Cheeger-Chern-Simons class. In this subsection we review the definition of the Cheeger-Chern-Simons class of complex flat vector bundles. We refer to [11, 17, 27] for the details.

Let $F \rightarrow X$ be a complex flat vector bundle with flat connection $\nabla^{F}$. The Cheeger-Chern-Simons class $\operatorname{CCS}\left(F, \nabla^{F}\right) \in H^{\text {odd }}(X ; \mathbb{C} / \mathbb{Q})$ of $\left(F, \nabla^{F}\right)$ [11, $\S 1(\mathrm{~g})$ ] (see also [27, Definition 2.11]) is defined as follows. Denote by $\mathbb{C}^{N} \rightarrow X$ the trivial complex vector bundle of rank $N$. Since $\operatorname{ch}([F]-$ $\left.\left[\mathbb{C}^{\operatorname{rank}(F)}\right]\right)=0 \in H^{\text {even }}(X ; \mathbb{Q})$, by $[2$, p.89] there exists $k \in \mathbb{N}$ such that $k F \cong k \mathbb{C}^{\operatorname{rank}(F)}$ as smooth complex vector bundles.

Remark 1. This fact can be proved as follows. Let $E_{1} \rightarrow X$ and $E_{2} \rightarrow X$ be complex vector bundles of the same rank $\ell$. Recall from [21, Theorem 1.5 of Chapter 9] that if $2 \ell \geq \operatorname{dim}(X)$ and $E_{1} \oplus \mathbb{C}^{m} \cong E_{2} \oplus \mathbb{C}^{m}$ for some $m \in \mathbb{N}$, then $E_{1} \cong E_{2}$.

Now suppose $E_{1} \rightarrow X$ and $E_{2} \rightarrow X$ have the same rank $\ell$ and satisfy $\operatorname{ch}\left(\left[E_{1}\right]-\left[E_{2}\right]\right)=0$. Since $\operatorname{ch}: K^{0}(X) \otimes \mathbb{Q} \rightarrow H^{\text {even }}(X ; \mathbb{Q})$ is a ring isomorphism, there exists $k \in \mathbb{N}$ such that $k\left[E_{1}\right]=k\left[E_{2}\right]$. Since it also holds for any integer multiple of $k$, we can take a sufficiently large integer multiple of $k$, still denoted by $k$, so that $2 k \ell \geq \operatorname{dim}(X)$. Since $k E_{1} \oplus \mathbb{C}^{m} \cong k E_{2} \oplus \mathbb{C}^{m}$ for some $m \in \mathbb{N}$, it follows from [21, Theorem 1.5 of Chapter 9] that $k E_{1} \cong k E_{2}$. 
Let $\nabla_{0}^{k F}$ be a trivial connection on $k F \rightarrow X$, which can be determined by choosing a global frame for $k F \rightarrow X$. One can check that the odd form $\frac{1}{k} \operatorname{CS}\left(\nabla_{0}^{k F}, k \nabla^{F}\right)$ is closed. The Cheeger-Chern-Simons class of $\left(F, \nabla^{F}\right)$ is defined to be

$$
\operatorname{CCS}\left(F, \nabla^{F}\right):=\left[\frac{1}{k} \operatorname{CS}\left(\nabla_{0}^{k F}, k \nabla^{F}\right)\right] \bmod \mathbb{Q} .
$$

Note that $\operatorname{CCS}\left(F, \nabla^{F}\right)$ is independent of the choices of $k \in \mathbb{N}$ such that $k F \cong k \mathbb{C}^{\operatorname{rank}(F)}\left[26\right.$, Theorem B.5.4] and $\nabla_{0}^{k F}$ (by proceeding as in [25, Lemma 1]).

Put a Hermitian metric $g^{F}$ on $F \rightarrow X$. As in [12, Definition 4.1] define

$$
\omega\left(F, g^{F}\right):=\left(g^{F}\right)^{-1}\left(\nabla^{F} g^{F}\right) \in \Omega^{1}(X, \operatorname{End}(F)) .
$$

By [12, Definition 4.2, Proposition 4.3], the connection $\nabla^{F, u}$ on $F \rightarrow X$ defined by

$$
\nabla^{F, u}=\nabla^{F}+\frac{1}{2} \omega\left(F, g^{F}\right)
$$

is unitary with respect to $g^{F}$ and has curvature

$$
\left(\nabla^{F, u}\right)^{2}=-\frac{1}{4} \omega\left(F, g^{F}\right)^{2} .
$$

By [7, $(2.33),(2.37)], \operatorname{tr}\left(\omega\left(F, g^{F}\right)^{2 k}\right)=0$ for any $k \in \mathbb{N}$, and therefore

$$
\operatorname{ch}\left(\nabla^{F, u}\right)=\operatorname{rank}(F) .
$$

By [27, (2.44)] the real part of $\operatorname{CCS}\left(F, \nabla^{F}\right)$ is given by

$$
\operatorname{Re}\left(\operatorname{CCS}\left(F, \nabla^{F}\right)\right)=\left[\frac{1}{k} \operatorname{CS}\left(\nabla_{0}^{k F}, k \nabla^{F, u}\right)\right] \bmod \mathbb{Q} \in H^{\text {odd }}(X ; \mathbb{R} / \mathbb{Q}) .
$$

Remark 2. The Cheeger-Chern-Simons class $\operatorname{CCS}\left(F, \nabla^{F}\right)$ measures the deviation of $\nabla^{F}$ (or more precisely its $k$-fold direct sum) from being a trivial connection. If $\nabla^{F}$ (or $k \nabla^{F}$ ) is indeed a trivial connection, then $k \nabla^{F}$ differs from $\nabla_{0}^{k F}$ by a gauge transformation specified by a smooth map $g: X \rightarrow$ $\operatorname{GL}(k \operatorname{rank}(F) ; \mathbb{C})$. Thus $\operatorname{CS}\left(\nabla_{0}^{k F}, k \nabla^{F}\right)=\operatorname{ch}^{\text {odd }}(g) \in \Omega_{\mathbb{Q}}^{\text {odd }}(X ; \mathbb{C})$. Since $\operatorname{CCS}\left(F, \nabla^{F}\right)$ is the $\bmod \mathbb{Q}$ reduction of the de Rham class of a rational multiple of $\operatorname{CS}\left(\nabla_{0}^{k F}, k \nabla^{F}\right)$, it follows that $\operatorname{CCS}\left(F, \nabla^{F}\right)=0 \in H^{\text {odd }}(X ; \mathbb{C} / \mathbb{Q})$.

Let $(E, v)$ be a $\mathbb{Z}$-graded cochain complex of complex vector bundles over $X$, i.e.

$$
0 \longrightarrow E^{0} \stackrel{v}{\longrightarrow} E^{1} \stackrel{v}{\longrightarrow} \cdots \stackrel{v}{\longrightarrow} E^{m} \longrightarrow 0
$$

with $v \circ v=0$. Let $\nabla^{k}$ be a connection on $E^{k} \rightarrow X$ for each $0 \leq k \leq m$. Define

$$
E=\bigoplus_{k=0}^{m} E^{k} \quad \text { and } \quad \nabla^{E}=\bigoplus_{k=0}^{m} \nabla^{k}
$$


Note that $\nabla^{E}$ is a $\mathbb{Z}$-graded connection on $E \rightarrow X$. The triple $\left(E, v, \nabla^{E}\right)$ is called a flat cochain complex if $\nabla^{E}$ is a flat connection on $E \rightarrow X$ and $\left[\nabla^{E}, v\right]=0$. Define

$$
\begin{array}{ll}
E^{+}=\bigoplus_{k} E^{2 k}, & E^{-}=\bigoplus_{k} E^{2 k+1}, \\
\nabla^{+}=\bigoplus_{k} \nabla^{2 k}, & \nabla^{-}=\bigoplus_{k} \nabla^{2 k+1} .
\end{array}
$$

Then $E \rightarrow X$, where $E=E^{+} \oplus E^{-}$, is a $\mathbb{Z}_{2}$-graded complex vector bundle with a $\mathbb{Z}_{2}$-graded connection $\nabla^{E}=\nabla^{+} \oplus \nabla^{-}$.

For any $\mathbb{Z}$-graded cochain complex $(E, v)$ with two $\mathbb{Z}$-graded connections $\nabla_{1}^{E}$ and $\nabla_{0}^{E}$ define

$$
\begin{aligned}
\operatorname{ch}\left(E, \nabla_{j}^{E}\right) & :=\sum_{k=0}^{m}(-1)^{k} \operatorname{ch}\left(E^{k}, \nabla_{j}^{k}\right) \text { where } j=0,1, \\
\operatorname{CS}\left(\nabla_{1}^{E}, \nabla_{0}^{E}\right) & :=\sum_{k=0}^{m}(-1)^{k} \operatorname{CS}\left(\nabla_{1}^{k}, \nabla_{0}^{k}\right) .
\end{aligned}
$$

The Cheeger-Chern-Simons class of a $\mathbb{Z}$-graded flat cochain complex $\left(E, v, \nabla^{E}\right)$ is defined to be

$$
\operatorname{CCS}\left(E, \nabla^{E}\right)=\sum_{k=0}^{m}(-1)^{k} \operatorname{CCS}\left(E^{k}, \nabla^{k}\right) \in H^{\text {odd }}(X ; \mathbb{C} / \mathbb{Q}) .
$$

Let $F \rightarrow X$ be a $\mathbb{Z}_{2}$-graded complex flat vector bundle with $\mathbb{Z}_{2}$-graded flat connection $\nabla^{F}=\nabla^{+} \oplus \nabla^{-}$of virtual rank zero. Since $\operatorname{ch}\left(\nabla^{+}\right)-\operatorname{ch}\left(\nabla^{-}\right)=$ $\operatorname{rank}\left(F^{+}\right)-\operatorname{rank}\left(F^{-}\right)=0$, there exist $k \in \mathbb{N}$ and a smooth bundle isomorphism $j: k F^{+} \rightarrow k F^{-}$. The Cheeger-Chern-Simons class of $\left(F, \nabla^{F}\right)$ is given by

$$
\operatorname{CCS}\left(F, \nabla^{F}\right)=\left[\frac{1}{k} \operatorname{CS}\left(j^{*} k \nabla^{-}, k \nabla^{+}\right)\right] \bmod \mathbb{Q} .
$$

Note that $\operatorname{CCS}\left(F, \nabla^{F}\right)$ is independent of the choices of $k$ [26, Theorem B.5.4] and $j$.

Remark 3. To prove that $\operatorname{CCS}\left(F, \nabla^{F}\right)$ is independent of the choice of $j$, suppose $j_{1}: k F^{+} \rightarrow k F^{-}$is another smooth bundle isomorphism. By (2.1.8) and 2.1 .9 we have

$$
\begin{aligned}
\mathrm{CS}\left(j^{*} k \nabla^{-}, k \nabla^{+}\right)-\operatorname{CS}\left(j_{1}^{*} k \nabla^{-}, k \nabla^{+}\right) & =\operatorname{CS}\left(j^{*} k \nabla^{-}, k \nabla^{+}\right)+\operatorname{CS}\left(k \nabla^{+}, j_{1}^{*} k \nabla^{-}\right) \\
& =\operatorname{CS}\left(j^{*} k \nabla^{-}, j_{1}^{*} k \nabla^{-}\right) \\
& =\operatorname{CS}\left(k \nabla^{-},\left(j^{-1}\right)^{*} j_{1}^{*} k \nabla^{-}\right) \\
& =\operatorname{CS}\left(k \nabla^{-},\left(j_{1} \circ j^{-1}\right)^{*} k \nabla^{-}\right),
\end{aligned}
$$


where the third equality follows from the fact that $j$ covers the identity map $\operatorname{id}_{X}$. Since $j_{1} \circ j^{-1} \in \operatorname{Aut}\left(k F^{-}\right)$, it follows that $\left(k F^{-}, j_{1} \circ j^{-1}\right)$ defines an element in $K^{-1}(X)$ [22, p.71-p.73]. It is well known that for any $[(E, f)] \in K^{-1}(X)$, a differential form representative of the odd Chern character $\operatorname{ch}^{\text {odd }}([(E, f)]) \in H^{\text {odd }}(X ; \mathbb{Q})$ is given by $\operatorname{CS}\left(\nabla^{E}, f^{*} \nabla^{E}\right)$, where $\nabla^{E}$ is any connection on $E \rightarrow X$ (see [17, p.955] for its Hermitian analog). Thus

$\mathrm{CS}\left(j^{*} k \nabla^{-}, k \nabla^{+}\right)-\operatorname{CS}\left(j_{1}^{*} k \nabla^{-}, k \nabla^{+}\right)=\operatorname{CS}\left(k \nabla^{-},\left(j_{1} \circ j^{-1}\right)^{*} k \nabla^{-}\right) \in \Omega_{\mathbb{Q}}^{\text {odd }}(X ; \mathbb{C})$.

To obtain the formula of $\operatorname{Re}\left(\operatorname{CCS}\left(F, \nabla^{F}\right)\right)$, put a $\mathbb{Z}_{2}$-graded Hermitian metric $g^{F}=g^{+} \oplus g^{-}$on $F \rightarrow X$, and define unitary connections $\nabla^{ \pm, u}$ on $F^{ \pm} \rightarrow X$ with respect to $g^{ \pm}$by (2.2.3). Since $k g^{+}$and $j^{*} k g^{-}$are Hermitian metrics on $k F^{+} \rightarrow X$, by the proof of [22, Theorem 8.8 of Chapter 1] there exists $f \in \operatorname{Aut}\left(k F^{+}\right)$such that

$$
k g^{+}=f^{*} j^{*} k g^{-}=(j \circ f)^{*} k g^{-} .
$$

Write $\tilde{j}$ for $j \circ f$. Note that $\tilde{j}^{*} k \nabla^{-, u}$ is unitary with respect to $k g^{+}$. Since $\widetilde{j}$ covers the identity $\operatorname{map~id}_{X}$, it follows from 2.1.9) and 2.1.10 that

$$
\begin{aligned}
& \operatorname{CS}\left(\widetilde{j}^{*} k \nabla^{-}, k \nabla^{+}\right) \\
= & \operatorname{CS}\left(\widetilde{j}^{*} k \nabla^{-}, \widetilde{j}^{*} k \nabla^{-, u}\right)+\operatorname{CS}\left(\widetilde{j}^{*} k \nabla^{-, u}, k \nabla^{+, u}\right)+\operatorname{CS}\left(k \nabla^{+, u}, k \nabla^{+}\right) \\
= & \operatorname{CS}\left(k \nabla^{-}, k \nabla^{-, u}\right)+\operatorname{CS}\left(\widetilde{j}^{*} k \nabla^{-, u}, k \nabla^{+, u}\right)+\operatorname{CS}\left(k \nabla^{+, u}, k \nabla^{+}\right) .
\end{aligned}
$$

Since $i \operatorname{Im}\left(\operatorname{CCS}\left(F^{ \pm}, \nabla^{ \pm}\right)\right)=\operatorname{CS}\left(\nabla^{ \pm, u}, \nabla^{ \pm}\right)$by [27, (2.43)], it follows from 2.2 .10 and 2.2 .12 that

$$
\operatorname{Re}\left(\operatorname{CCS}\left(F, \nabla^{F}\right)\right)=\left[\frac{1}{k} \operatorname{CS}\left(\widetilde{j}^{*} k \nabla^{-, u}, k \nabla^{+, u}\right)\right] \bmod \mathbb{Q} .
$$

It is necessary to define $\operatorname{Re}\left(\operatorname{CCS}\left(F, \nabla^{F}\right)\right)$ in terms of $\widetilde{j}^{*} k \nabla^{-, u}$ instead of $j^{*} k \nabla^{-, u}$. Since the connection $j^{*} k \nabla^{-, u}$ is not unitary with respect to $k g^{+}$ in general, if it was used to define $\operatorname{Re}\left(\operatorname{CCS}\left(F, \nabla^{F}\right)\right)$ then the Chern-Simons form on the right-hand side of (2.2.13) would not be real-valued.

Since $\operatorname{CCS}\left(F, \nabla^{F}\right)$ is independent of the choices of $k \in \mathbb{N}$ and $j: k F^{+} \rightarrow$ $k F^{-}$, and $f$ is uniquely determined by $\left(k, j, g^{F}\right), \operatorname{Re}\left(\operatorname{CCS}\left(F, \nabla^{F}\right)\right)$ is independent of the choices made as well.

2.3. Local index theory for twisted de Rham operator. In this subsection we recall the setup and the statement of the local FIT for twisted de Rham operator [7, §3] (see also [11, 27]).

Let $\pi: X \rightarrow B$ be a submersion with closed fibers $Z$ of dimension $n$. Denote by $T^{V} X \rightarrow X$ the vertical tangent bundle. Let $T^{H} X \rightarrow X$ be a horizontal distribution for $\pi: X \rightarrow B$, i.e. $T X=T^{V} X \oplus T^{H} X$. Denote by $P^{T^{V} X}: T X \rightarrow T^{V} X$ the projection map. Put a metric $g^{T^{V} X}$ on $T^{V} X \rightarrow X$. Given a Riemannian metric $g^{T B}$ on $T B \rightarrow B$, define a metric on $T X \rightarrow X$ by

$$
g^{T X}:=g^{T^{V} X} \oplus \pi^{*} g^{T B} .
$$


If $\nabla^{T X}$ is the corresponding Levi-Civita connection on $T X \rightarrow X$, then $\nabla^{T^{V} X}:=P^{T^{V} X} \nabla^{T X}$ is an Euclidean connection on $T^{V} X \rightarrow X$ with respect to $g^{T^{V} X}$.

The exterior bundle $\Lambda\left(T^{V} X\right)^{*} \rightarrow X$ is a Clifford module with Clifford multiplication $c(Y)=\varepsilon(Y)-i(Y)$, where $Y \in \Gamma\left(X, T^{V} X\right), \varepsilon$ is the exterior multiplication and $i$ is the interior multiplication. Here $T^{V} X \rightarrow X$ is identified with $\left(T^{V} X\right)^{*} \rightarrow X$ via $g^{T^{V} X}$. Denote by $\nabla^{\Lambda\left(T^{V} X\right)^{*}}$ the extension of $\nabla^{T^{V} X}$ on $T^{V} X \rightarrow X$ to $\Lambda\left(T^{V} X\right)^{*} \rightarrow X$. Set

$$
\widehat{c}(Y)=\varepsilon(Y)+i(Y) .
$$

Let $F \rightarrow X$ be a complex flat vector bundle with flat connection $\nabla^{F}$. Put a Hermitian metric $g^{F}$ on $F \rightarrow X$. Define a twisted Dirac operator $\mathrm{D}^{\Lambda \otimes F}: \Gamma\left(X, \Lambda\left(T^{V} X\right)^{*} \otimes F\right) \rightarrow \Gamma\left(X, \Lambda\left(T^{V} X\right)^{*} \otimes F\right)$ by

$$
\mathrm{D}^{\Lambda \otimes F}=\sum_{k=1}^{n} c\left(e_{k}\right) \nabla_{e_{k}}^{\Lambda\left(T^{V} X\right)^{*} \otimes F, u},
$$

where $\nabla^{\Lambda\left(T^{V} X\right)^{*} \otimes F, u}$ is the tensor product of $\nabla^{\Lambda\left(T^{V} X\right)^{*}}$ and $\nabla^{F, u}$, and $\left\{e_{k}\right\}$ is a local orthonormal frame for $T^{V} X \rightarrow X$. Define an infinite rank $\mathbb{Z}$-graded complex vector bundle $\pi_{*}^{\Lambda} F \rightarrow B$ whose fiber over $b \in B$ is

$$
\left(\pi_{*}^{\Lambda} F\right)_{b}:=\Gamma\left(Z_{b},\left.\left(\Lambda\left(T^{V} X\right)^{*} \otimes F\right)\right|_{Z_{b}}\right) .
$$

By [11, (3.6)] we have

$$
\Omega(X, F) \cong \Omega\left(B, \pi_{*}^{\Lambda} F\right) .
$$

Denote by $*$ the fiberwise Hodge star operator associated to $g^{T^{V} X}$, and extend it from $\Gamma\left(X, \Lambda\left(T^{V} X\right)^{*}\right)$ to $\Gamma\left(X, \Lambda\left(T^{V} X\right)^{*} \otimes F\right) \cong \Gamma\left(B, \pi_{*}^{\Lambda} F\right)$. Define an $L^{2}$-metric on $\pi_{*}^{\Lambda} F \rightarrow B$ by

$$
g^{\pi_{*}^{\Lambda} F}\left(s_{1}, s_{2}\right)(b)=\int_{Z_{b}} g^{F}\left(s_{1}(b) \wedge * s_{2}(b)\right) .
$$

Let $U \in \Gamma(B, T B)$ and denote by $U^{H} \in \Gamma\left(X, T^{H} X\right)$ its lift. Define a connection $\nabla^{\pi_{*}^{\Lambda} F}$ on $\pi_{*}^{\Lambda} F \rightarrow B$ by

$$
\nabla_{U}^{\pi_{*}^{\Lambda} F} s=\nabla_{U^{H}}^{\Lambda\left(T^{V} X\right)^{*} \otimes F, u} s
$$

where $s \in \Gamma\left(B, \pi_{*}^{\Lambda} F\right)$. Note that $\nabla^{\pi_{*}^{\Lambda} F}$ preserves the $\mathbb{Z}$-grading of $\pi_{*}^{\Lambda} F \rightarrow B$. Denote by $\nabla^{T B}$ the Levi-Civita connection associated to $g^{T B}$, and define ${ }^{0} \nabla^{T X}=\pi^{*} \nabla^{T B} \oplus \nabla^{T^{V} X}$. Define $S:=\nabla^{T X}{ }^{0} \nabla^{T X} \in \Omega^{1}(X, \operatorname{End}(T X))$. By [5, Theorem 1.9], the $(3,0)$ tensor $g^{T X}(S(\cdot) \cdot, \cdot)$ only depends on $\left(T^{H} X, g^{T^{V} X}\right)$. Define a horizontal one-form $k$ on $X$ by

$$
k\left(U^{H}\right)=-\sum_{k=1}^{n} g^{T X}\left(S\left(e_{k}\right) e_{k}, U^{H}\right) .
$$


The connection $\nabla^{\pi_{*}^{\Lambda} F, u}$ on $\pi_{*}^{\Lambda} F \rightarrow B$ defined by

$$
\nabla^{\pi_{*}^{\Lambda} F, u}:=\nabla^{\pi_{*}^{\Lambda} F}+\frac{1}{2} k
$$

is unitary with respect to $g^{\pi_{*}^{\Lambda} F}$ [9, Proposition 1.4].

Denote by $d^{Z}$ the fiberwise de Rham operator coupled with $\nabla^{F}$ acting on $\pi_{*}^{\Lambda} F \rightarrow B$. The connection $\widetilde{\nabla}^{\pi_{*}^{\Lambda} F}\left[11\right.$, Definition 3.2] on $\pi_{*}^{\Lambda} F \rightarrow B$ defined by

$$
\widetilde{\nabla}_{U}^{\pi_{*}^{\Lambda} F} s:=\mathcal{L}_{U^{H}} s
$$

where $U \in \Gamma(B, T B), U^{H} \in \Gamma\left(X, T^{H} X\right)$ is its lift, $\mathcal{L}_{U^{H}}: \Gamma\left(X, \Lambda\left(T^{V} X\right)^{*} \otimes\right.$ $F) \rightarrow \Gamma\left(X, \Lambda\left(T^{V} X\right)^{*} \otimes F\right)$ is the Lie derivative and $s \in \Gamma\left(B, \pi_{*}^{\Lambda} F\right)$, is $\mathbb{Z}$ graded.

The exterior differential $d^{X}: \Omega(X, F) \rightarrow \Omega(X, F)$ coupled with $\nabla^{F}$ can be regarded as a flat superconnection on $\pi_{*}^{\Lambda} F \rightarrow B$ of total degree 1 whose decomposition [11, Proposition 3.4] is given by

$$
d^{X}=d^{Z}+\widetilde{\nabla}^{\pi_{*}^{\Lambda} F}+i_{T}
$$

where $T$ is the curvature 2 -form of the fiber bundle $X \rightarrow B$, and $i_{T}$ is given in [11, Definition 3.3].

Consider $d^{Z}$ as an element in $\Gamma\left(B, \operatorname{Hom}\left(\left(\pi_{*}^{\Lambda} F\right)^{\bullet},\left(\pi_{*}^{\Lambda} F\right)^{\bullet+1}\right)\right)$. For each $b \in B$,

$$
0 \longrightarrow\left(\pi_{*}^{\Lambda} F\right)_{b}^{0} \stackrel{d^{Z_{b}}}{\longrightarrow}\left(\pi_{*}^{\Lambda} F\right)_{b}^{1} \stackrel{d^{Z_{b}}}{\longrightarrow} \cdots \stackrel{d^{Z_{b}}}{\longrightarrow}\left(\pi_{*}^{\Lambda} F\right)_{b}^{n} \longrightarrow 0
$$

is a cochain complex. Denote by $H^{k}\left(Z_{b},\left.F\right|_{Z_{b}}\right)$ the associated $k$-th cohomology group and define $H\left(Z_{b},\left.F\right|_{Z_{b}}\right):=\bigoplus_{k=0}^{n} H^{k}\left(Z_{b},\left.F\right|_{Z_{b}}\right)$. Define a $\mathbb{Z}$-graded complex vector bundle $H\left(Z,\left.F\right|_{Z}\right) \rightarrow B$ whose fiber over $b \in B$ is given by $H\left(Z,\left.F\right|_{Z}\right)_{b}:=H\left(Z_{b},\left.F\right|_{Z_{b}}\right)$. Denote by $\psi: \operatorname{ker}\left(d^{Z}\right) \rightarrow H\left(Z,\left.F\right|_{Z}\right)$ the quotient map. For $s \in \Gamma\left(B, H^{k}\left(Z,\left.F\right|_{Z}\right)\right)$, let $e \in \Gamma\left(B,\left(\pi_{*}^{\Lambda} F\right)^{k} \cap \operatorname{ker}\left(d^{Z}\right)\right)$ be such that $\psi(e)=s$. The connection $\nabla^{H\left(Z,\left.F\right|_{Z}\right)}$ on $H\left(Z,\left.F\right|_{Z}\right) \rightarrow B$ [11, Definition 2.4] defined by

$$
\nabla_{U}^{H(Z, F \mid z)} s=\psi\left(\widetilde{\nabla}_{U}^{\pi_{*}^{\Lambda} F} e\right)
$$

where $U \in \Gamma(B, T B)$, is a well defined $\mathbb{Z}$-graded flat connection [11, Definition 2.4, Proposition 2.5].

Denote by $d^{Z *}$ the formal adjoint of $d^{Z}$ with respect to $g^{\pi_{*}^{\Lambda} F}$. As in [11, Definition 3.8] define

$$
\begin{aligned}
\mathrm{D}^{Z, \mathrm{dR}} & =d^{Z}+d^{Z *}, \\
\widetilde{\nabla}^{\pi_{*}^{\Lambda} F, u} & =\frac{1}{2}\left(\widetilde{\nabla}^{\pi_{*}^{\Lambda} F}+\left(\widetilde{\nabla}^{\pi_{*}^{\Lambda} F}\right)^{*}\right), \\
\omega\left(\pi_{*}^{\Lambda} F, g^{\pi_{*}^{\Lambda} F}\right) & =\left(\widetilde{\nabla}^{\pi_{*}^{\Lambda} F}\right)^{*}-\widetilde{\nabla}^{\pi_{*}^{\Lambda} F},
\end{aligned}
$$


where $\left(\widetilde{\nabla}^{\pi_{*}^{\Lambda} F}\right)^{*}$ is the adjoint of $\widetilde{\nabla}^{\pi_{*}^{\Lambda} F}$ with respect to $g^{\pi_{*}^{\Lambda} F}$ 11, Definition 1.6]. By Hodge theory we have

$$
H\left(Z_{b},\left.F\right|_{Z_{b}}\right) \cong \operatorname{ker}\left(\mathrm{D}^{Z_{b}, \mathrm{dR}}\right) .
$$

Define a $\mathbb{Z}$-graded complex vector bundle $\operatorname{ker}\left(\mathrm{D}^{Z, \mathrm{dR}}\right) \rightarrow B$ whose fiber over $b \in B$ is given by $\operatorname{ker}\left(\mathrm{D}^{Z, \mathrm{dR}}\right)_{b}:=\operatorname{ker}\left(\mathrm{D}^{Z_{b}, \mathrm{dR}}\right)$. Then $\operatorname{ker}\left(\mathrm{D}^{Z, \mathrm{dR}}\right) \rightarrow B$ is a finite rank subbundle of $\pi_{*}^{\Lambda} F \rightarrow B$ and

$$
H\left(Z,\left.F\right|_{Z}\right) \cong \operatorname{ker}\left(\mathrm{D}^{Z, \mathrm{dR}}\right)
$$

as $\mathbb{Z}$-graded complex vector bundles. Note that $\operatorname{ker}\left(\mathrm{D}^{Z, \mathrm{dR}}\right) \rightarrow B$ inherits a $\mathbb{Z}$-graded Hermitian metric from $g^{\pi_{*}^{\Lambda} F}$, which will be denoted by $g^{\operatorname{ker}\left(\mathrm{D}^{Z, \mathrm{dR}}\right)}$. Denote by $g^{H\left(Z,\left.F\right|_{Z}\right)}$ the $\mathbb{Z}$-graded Hermitian metric on $H\left(Z,\left.F\right|_{Z}\right) \rightarrow B$ obtained by pulling back $g^{\operatorname{ker}\left(\mathrm{D}^{Z, \mathrm{dR}}\right)}$ via the isomorphism 2.3.5). Denote by $P^{\operatorname{ker}\left(\mathrm{D}^{Z, \mathrm{dR}}\right)}: \pi_{*}^{\Lambda} F \rightarrow \operatorname{ker}\left(\mathrm{D}^{Z, \mathrm{dR}}\right)$ the orthogonal projection onto $\operatorname{ker}\left(\mathrm{D}^{Z, \mathrm{dR}}\right)$. Then $P^{\operatorname{ker}\left(\mathrm{D}^{Z, \mathrm{dR}}\right)} \widetilde{\nabla}^{\pi_{*}^{\Lambda} F}$ is a connection on $\operatorname{ker}\left(\mathrm{D}^{Z, \mathrm{dR}}\right) \rightarrow B$, which can be considered as a connection on $H\left(Z,\left.F\right|_{Z}\right) \rightarrow B$ via the isomorphism 2.3.5. By [11, Proposition 3.14] we have

$$
\begin{aligned}
\nabla^{H\left(Z,\left.F\right|_{Z}\right)} & =P^{\mathrm{ker}\left(\mathrm{D}^{Z, \mathrm{dR}}\right)} \widetilde{\nabla}^{\pi_{*}^{\Lambda} F} \\
\omega\left(H\left(Z,\left.F\right|_{Z}\right), g^{H\left(Z,\left.F\right|_{Z}\right)}\right) & =P^{\operatorname{ker}\left(\mathrm{D}^{Z, \mathrm{dR}}\right)} \omega\left(\pi_{*}^{\Lambda} F, g^{\pi_{*}^{\Lambda} F}\right) P^{\operatorname{ker}\left(\mathrm{D}^{Z, \mathrm{dR}}\right)} .
\end{aligned}
$$

Define $\nabla^{H\left(Z,\left.F\right|_{Z}\right), u}:=\nabla^{H\left(Z,\left.F\right|_{Z}\right)}+\frac{1}{2} \omega\left(H\left(Z,\left.F\right|_{Z}\right), g^{H\left(Z,\left.F\right|_{Z}\right)}\right)$. Consequently we have

$$
\nabla^{H\left(Z,\left.F\right|_{Z}\right), u}=P^{\operatorname{ker}\left(\mathrm{D}^{Z, \mathrm{dR}}\right)} \widetilde{\nabla}_{*}^{\pi_{*}^{\Lambda} F, u} .
$$

By [11, (3.38)] we have

$$
\widetilde{\nabla}^{\pi_{*}^{\Lambda} F, u}=\nabla^{\pi_{*}^{\Lambda} F, u}
$$

where the left-hand side of $(2.3 .6)$ is defined by (2.2.3), and the right-hand side of 2.3.6 is defined by (2.3.4). Therefore we have

$$
\nabla^{H\left(Z,\left.F\right|_{Z}\right), u}=P^{\operatorname{ker}\left(\mathrm{D}^{Z, \mathrm{dR}}\right)} \nabla_{*}^{\pi_{*}^{\Lambda} F, u} .
$$

By [12, Proposition 4.12] we have

$$
\mathrm{D}^{Z, \mathrm{dR}}=\mathrm{D}^{\Lambda \otimes F}+V
$$

where

$$
V=-\frac{1}{2} \sum_{k=1}^{n} \widehat{c}\left(e_{k}\right) \omega\left(F, g^{F}\right)\left(e_{k}\right) .
$$

Note that $V$ is an odd self-adjoint matrix-valued operator which anti-commutes with the $c(X)$ 's.

Define the Bismut superconnection $\mathbb{B}^{\mathrm{dR}}$ on $\pi_{*}^{\Lambda} F \rightarrow B$ [7, (3.49)] associated to $\mathrm{D}^{Z, \mathrm{dR}}$ by

$$
\mathbb{B}^{\mathrm{dR}}:=\mathrm{D}^{Z, \mathrm{dR}}+\nabla^{\pi_{*}^{\Lambda} F, u}-\frac{c(T)}{4} .
$$


The rescaled Bismut superconnection $\mathbb{B}_{t}^{\mathrm{dR}}$ is given by

$$
\mathbb{B}_{t}^{\mathrm{dR}}:=\sqrt{t} \mathrm{D}^{Z, \mathrm{dR}}+\nabla^{\pi_{*}^{\Lambda} F, u}-\frac{c(T)}{4 \sqrt{t}} .
$$

One might consider the "unperturbed" Bismut superconnection $\mathbb{B}$ associated to $\mathrm{D}^{\Lambda \otimes F}$, i.e. $\mathbb{B}=\mathrm{D}^{\Lambda \otimes F}+\nabla^{\pi_{*}^{\Lambda} F, u}-\frac{c(T)}{4}$, instead of $\mathbb{B}^{\mathrm{dR}}$ defined by 2.3.10 whose degree zero term $\mathbb{B}_{[0]}^{\mathrm{dR}}$ is perturbed by $V$. The reason of considering $\mathbb{B}^{\mathrm{dR}}$ is because our target is the cohomology bundle $H\left(Z,\left.F\right|_{Z}\right) \rightarrow B$, which is isomorphic to $\operatorname{ker}\left(\mathrm{D}^{\Lambda \otimes F}+V\right) \rightarrow B$. Here the notion of superconnection is generalized in the sense of [4, p.286]. By [11, (3.40), (3.45), (3.46), (3.50)] and the proof of [11, Theorem 3.15], techniques of local index theory can still be applied to $\mathbb{B}^{\mathrm{dR}}$. See also the last paragraph of [7, p.33].

The Bismut-Cheeger eta form $\widetilde{\eta}^{\mathrm{dR}}$ associated to $\mathbb{B}^{\mathrm{dR}}$ is defined by

$$
\widetilde{\eta}^{\mathrm{dR}}:=\int_{0}^{\infty} \operatorname{str}\left(\frac{d \mathbb{B}_{t}^{\mathrm{dR}}}{d t} e^{-\frac{1}{2 \pi i}\left(\mathbb{B}_{t}^{\mathrm{dR}}\right)^{2}}\right) d t .
$$

For $\operatorname{dim}(Z)$ even, the local FIT for the twisted de Rham operator $\mathrm{D}^{Z, \mathrm{dR}}$ [11, Theorem 3.15] (see also [7, (3.50)]) is given by

$$
d \widetilde{\eta}^{\mathrm{dR}}=\int_{X / B} e\left(\nabla^{T^{V} X}\right) \wedge \operatorname{ch}\left(\nabla^{F, u}\right)-\operatorname{ch}\left(\nabla^{H\left(Z,\left.F\right|_{Z}\right), u}\right),
$$

where $e\left(\nabla^{T^{V} X}\right) \in \Omega_{\mathbb{Z}}^{n}\left(X ; o\left(T^{V} X\right)\right)$ is the $o\left(T^{V} X\right)$-valued Euler form. Here $o\left(T^{V} X\right) \rightarrow X$ is the orientation bundle of $T^{V} X \rightarrow X$. It is a flat real line bundle, which is trivial if and only if $T^{V} X \rightarrow X$ is oriented. Note that $e\left(\nabla^{T^{V} X}\right)=0$ if $n$ is odd. A priori 2.3.11) holds under the assumptions that the fibers $Z$ are oriented and spin. However, as noted in the proof of [11, Theorem 3.15], the computations involved are local, so (2.3.11) is still valid if the fibers are not spin and not even orientable.

By (2.2.5) we have

$$
\begin{aligned}
& \int_{X / B} e\left(\nabla^{T^{V} X}\right) \wedge \operatorname{ch}\left(\nabla^{F, u}\right)-\operatorname{ch}\left(\nabla^{H(Z, F \mid Z), u}\right) \\
= & \operatorname{rank}(F) \chi(Z)-\operatorname{rank}\left(H\left(Z,\left.F\right|_{Z}\right)\right)=0,
\end{aligned}
$$

where $\chi(Z): B \rightarrow \mathbb{Z}$ is the Euler characteristic function. Thus $\widetilde{\eta}^{\mathrm{dR}}$ is a closed form. A stronger result by Bismut [7, Theorem 3.7] (see also [11, (3.74)]) states that

$$
\widetilde{\eta}^{\mathrm{dR}}=0 .
$$

2.4. Local index theory for twisted spin Dirac operator. In this subsection we review the setup and the statement of the local FIT for twisted spin Dirac operator under and without the kernel bundle assumption. We refer to [25, $\S 5]$ and [17, $\S 7]$ for the details.

Let $\pi: X \rightarrow B$ be a submersion with closed, oriented and spin fibers $Z$ of even dimension $n$. Endow $T^{V} X \rightarrow X$ the geometric data as in Section 
2.3. Denote by $S\left(T^{V} X\right) \rightarrow X$ the $\mathbb{Z}_{2}$-graded spinor bundle. The connection $\nabla^{T^{V}} X$ on $T^{V} X \rightarrow X$ lifts uniquely to $S\left(T^{V} X\right) \rightarrow X$ and preserves its grading.

Let $E \rightarrow X$ be a complex vector bundle with a Hermitian metric $g^{E}$ and a unitary connection $\nabla^{E}$. Then $S\left(T^{V} X\right) \otimes E \rightarrow X$ is a $\mathbb{Z}_{2}$-graded complex vector bundle equipped with the Hermitian metric $g^{T^{V}} X \otimes g^{E}$ and the unitary connection $\nabla^{S\left(T^{V} X\right) \otimes E}$, which is defined to be the tensor product of $\nabla^{S\left(T^{V} X\right)}$ and $\nabla^{E}$. The twisted spin Dirac operator $\mathrm{D}^{S \otimes E}$ acting on $\Gamma\left(X, S\left(T^{V} X\right) \otimes E\right)$ is defined to be

$$
\mathrm{D}^{S \otimes E}=\sum_{k=1}^{n} c\left(e_{k}\right) \nabla_{e_{k}}^{S\left(T^{V} X\right) \otimes E},
$$

where $c$ is the Clifford multiplication, and $\left\{e_{k}\right\}$ is a local orthonormal frame for $T^{V} X \rightarrow X$.

Define an infinite rank $\mathbb{Z}_{2}$-graded complex vector bundle $\pi_{*}^{\text {spin }} E \rightarrow B$ whose fibers over $b \in B$ is given by

$$
\left(\pi_{*}^{\text {spin }} E\right)_{b}:=\Gamma\left(Z_{b},\left.\left(S\left(T^{V} X\right) \otimes E\right)\right|_{Z_{b}}\right) .
$$

Denote by $g^{\pi_{*}^{\text {spin }} E}$ the $L^{2}$-metric on $\pi_{*}^{\text {spin }} E \rightarrow B$ [7, (1.11)]. Define a connection $\nabla_{*}^{\pi_{*}^{\text {spin }} E}$ on $\pi_{*}^{\text {spin }} E \rightarrow B$ by

$$
\nabla_{U}^{\pi_{*}^{\text {spin }} E} s:=\nabla_{U^{H}}^{S\left(T^{V} X\right) \otimes E} s
$$

where $s \in \Gamma\left(B, \pi_{*}^{\text {spin }} E\right), U \in \Gamma(B, T B)$ and $U^{H} \in \Gamma\left(X, T^{H} X\right)$ is a lift of $U$. The connection $\nabla^{\pi_{*}^{\text {spin }} E, u}$ on $\pi_{*}^{\text {spin }} E \rightarrow B$ defined by

$$
\nabla_{*}^{\pi_{*}^{\text {spin }} E, u}:=\nabla_{*}^{\pi_{*}^{\text {spin }} E}+\frac{1}{2} k,
$$

where $k$ is given by 2.3 .3 , is $\mathbb{Z}_{2}$-graded and unitary with respect to $g^{\pi_{*}^{\text {spin }} E}$ [9, Proposition 1.4].

Assumption 1. The family of complex vector spaces $\operatorname{ker}\left(\mathrm{D}_{b}^{S \otimes E}\right), b \in B$, has locally constant dimension.

If Assumption 1 is satisfied, then the family of complex vector spaces $\operatorname{ker}\left(\mathrm{D}_{b}^{S \otimes E}\right)$ form a finite rank $\mathbb{Z}_{2}$-graded subbundle of $\pi_{*}^{\text {spin }} E \rightarrow B$, denoted by $\operatorname{ker}\left(\mathrm{D}^{S \otimes E}\right) \rightarrow B$. In this case the family of complex vector spaces $\operatorname{Im}\left(\mathrm{D}_{b}^{S \otimes E}\right)$ form an infinite rank $\mathbb{Z}_{2}$-graded subbundle of $\pi_{*}^{\text {spin }} E \rightarrow B$ as well, denoted by $\operatorname{Im}\left(\mathrm{D}^{S \otimes E}\right) \rightarrow B$. Their $\mathbb{Z}_{2}$-gradings are given by

$$
\operatorname{ker}\left(\mathrm{D}^{S \otimes E}\right)^{ \pm}=\operatorname{ker}\left(\mathrm{D}_{ \pm}^{S \otimes E}\right), \quad \operatorname{Im}\left(\mathrm{D}^{S \otimes E}\right)^{ \pm}=\operatorname{Im}\left(\mathrm{D}_{\mp}^{S \otimes E}\right) .
$$

Note that the direct sum decompositions

$$
\begin{aligned}
& \left(\pi_{*}^{\text {spin }} E\right)^{+}=\operatorname{Im}\left(\mathrm{D}^{S \otimes E}\right)^{+} \oplus \operatorname{ker}\left(\mathrm{D}^{S \otimes E}\right)^{+}, \\
& \left(\pi_{*}^{\text {spin }} E\right)^{-}=\operatorname{Im}\left(\mathrm{D}^{S \otimes E}\right)^{-} \oplus \operatorname{ker}\left(\mathrm{D}^{S \otimes E}\right)^{-}
\end{aligned}
$$

are orthogonal. The $K$-theoretic analytic index of $[E] \in K(X)$ can be defined as ind ${ }^{\mathrm{a}}([E])=\left[\operatorname{ker}\left(\mathrm{D}^{S \otimes E}\right)^{+}\right]-\left[\operatorname{ker}\left(\mathrm{D}^{S \otimes E}\right)^{-}\right]$. 
The Bismut superconnection $\mathbb{B}^{E}$ on $\pi_{*}^{\text {spin }} E \rightarrow B$ is defined to be

$$
\mathbb{B}^{E}=\mathrm{D}^{S \otimes E}+\nabla^{\pi_{*}^{\mathrm{spin}} E, u}-\frac{c(T)}{4}
$$

where $T$ is the curvature 2 -form of the fiber bundle $\pi: X \rightarrow B$. The rescaled Bismut superconnection $\mathbb{B}_{t}^{E}$ is given by

$$
\mathbb{B}_{t}^{E}=\sqrt{t} \mathrm{D}^{S \otimes E}+\nabla^{\pi_{*}^{\text {spin }} E, u}-\frac{c(T)}{4 \sqrt{t}} .
$$

Denote by $P^{\mathrm{ker}\left(\mathrm{D}^{S \otimes E}\right)}: \pi_{*}^{\text {spin }} E \rightarrow \operatorname{ker}\left(\mathrm{D}^{S \otimes E}\right)$ the orthogonal projection. Then $g^{\operatorname{ker}\left(\mathrm{D}^{S \otimes E}\right)}:=P^{\operatorname{ker}\left(\mathrm{D}^{S \otimes E}\right)} g^{\pi_{*}^{\text {spin }} E}$ is a Hermitian metric on $\operatorname{ker}\left(\mathrm{D}^{S \otimes E}\right) \rightarrow$ B. Moreover, $\nabla^{\operatorname{ker}\left(\mathrm{D}^{S \otimes E}\right)}:=P^{\operatorname{ker}\left(\mathrm{D}^{S \otimes E}\right)} \nabla^{\pi_{*}^{\text {spin }} E, u}$ is a $\mathbb{Z}_{2}$-graded unitary connection on $\operatorname{ker}\left(\mathrm{D}^{S \otimes E}\right) \rightarrow B$. Then

$$
\begin{aligned}
\lim _{t \rightarrow 0} \operatorname{ch}\left(\mathbb{B}_{t}^{E}\right) & =\int_{X / B} \widehat{A}\left(\nabla^{T^{V} X}\right) \wedge \operatorname{ch}\left(\nabla^{E}\right), \\
\lim _{t \rightarrow \infty} \operatorname{ch}\left(\mathbb{B}_{t}^{E}\right) & =\operatorname{ch}\left(\nabla^{\operatorname{ker}\left(\mathrm{D}^{S \otimes E}\right)}\right) .
\end{aligned}
$$

The Bismut-Cheeger eta form [8, 16] associated to $\mathbb{B}^{E}$ is defined to be

$$
\widetilde{\eta}^{E}\left(g^{E}, \nabla^{E}, T^{H} X, g^{T^{V} X}\right):=\int_{0}^{\infty} \operatorname{str}\left(\frac{d \mathbb{B}_{t}^{E}}{d t} e^{-\frac{1}{2 \pi i}\left(\mathbb{B}_{t}^{E}\right)^{2}}\right) d t .
$$

The notation for the Bismut-Cheeger eta form, inspired by Liu [24], is to emphasize the dependence on the geometric data involved.

The local FIT for $\mathrm{D}^{S \otimes E}$ states that

$$
d \widetilde{\eta}^{E}\left(g^{E}, \nabla^{E}, T^{H} X, g^{T^{V} X}\right)=\int_{X / B} \widehat{A}\left(\nabla^{T^{V} X}\right) \wedge \operatorname{ch}\left(\nabla^{E}\right)-\operatorname{ch}\left(\nabla^{\operatorname{ker}\left(\mathrm{D}^{S \otimes E}\right)}\right) .
$$

Now suppose Assumption 11 is not satisfied. Recall that $B$ is assumed to be closed. Miščenko-Fomenko [28] (see also [17, Lemma 7.13]) prove that there exist finite rank subbundles $L^{ \pm} \rightarrow B$ and complementary closed subbundles $K^{ \pm} \rightarrow B$ of $\left(\pi_{*}^{\text {spin }} E\right)^{ \pm} \rightarrow B$ such that

$$
\left(\pi_{*}^{\text {spin }} E\right)^{+}=K^{+} \oplus L^{+}, \quad\left(\pi_{*}^{\text {spin }} E\right)^{-}=K^{-} \oplus L^{-},
$$

$\mathrm{D}_{+}^{S \otimes E}:\left(\pi_{*}^{\text {spin }} E\right)^{+} \rightarrow\left(\pi_{*}^{\text {spin }} E\right)^{-}$is block diagonal as a map with respect to the direct sum decomposition 2.4 .9 , and $\left.\mathrm{D}_{+}^{S \otimes E}\right|_{K^{+}}: K^{+} \rightarrow K^{-}$is a smooth bundle isomorphism. Note that the subbundle $K^{+} \rightarrow B$ is not necessarily orthogonal to $L^{+} \rightarrow B$, and the same is true for $K^{-} \rightarrow B$ and $L^{-} \rightarrow B$.

Given $L^{ \pm} \rightarrow B$ satisfying the above conditions, we call the $\mathbb{Z}_{2}$-graded complex vector bundle $L \rightarrow B$, defined by $L=L^{+} \oplus L^{-}$, satisfies the $\mathrm{MF}$ property with respect to $\mathrm{D}^{S \otimes E}$. If $L \rightarrow B$ is a $\mathbb{Z}_{2}$-graded complex vector bundle satisfying the MF property with respect to $\mathrm{D}^{S \otimes E}$, then the $K$-theoretic analytic index of $[E] \in K(X)$ is defined to be ind ${ }^{\mathrm{a}}([E])=\left[L^{+}\right]-$ $\left[L^{-}\right] \in K(B)$. It is proved in $\left[28\right.$, p.96-97] that the definition of $\operatorname{ind}^{\mathrm{a}}([E])$ 
does not depend on the choice of $L \rightarrow B$ satisfying the MF property with respect to $\mathrm{D}^{S \otimes E}$.

Given a $\mathbb{Z}_{2}$-graded complex vector bundle $L \rightarrow B$ satisfying the MF property with respect to $\mathrm{D}^{S \otimes E}$, define an infinite rank bundle $\widetilde{\pi_{*}^{\text {spin }}} E \rightarrow B$ by

$$
\begin{aligned}
& \left(\widetilde{\pi_{*}^{\text {spin }}} E\right)^{+}:=\left(\pi_{*}^{\text {spin }} E\right)^{+} \oplus L^{-}, \\
& \left(\widetilde{\pi_{*}^{\text {spin }}} E\right)^{-}:=\left(\pi_{*}^{\text {spin }} E\right)^{-} \oplus L^{+} .
\end{aligned}
$$

That is, $\widetilde{\pi_{*}^{\text {spin }}} E:=\pi_{*}^{\text {spin }} E \oplus L^{\text {op }}$ as $\mathbb{Z}_{2}$-graded complex vector bundles. Let $i^{-}: L^{-} \rightarrow\left(\pi_{*}^{\text {spin }} E\right)^{-}$be the inclusion map and $p^{+}:\left(\pi_{*}^{\text {spin }} E\right)^{+} \rightarrow L^{+}$the projection map with respect to 2.4 .9 . For a given $\alpha \in \mathbb{C}$, define a map $\widetilde{\mathrm{D}}_{+}^{S \otimes E}(\alpha):\left(\widetilde{\pi_{*}^{\text {spin }}} E\right)^{+} \rightarrow\left(\widetilde{\pi_{*}^{\text {spin }}} E\right)^{-}$by

$$
\widetilde{\mathrm{D}}_{+}^{S \otimes E}(\alpha)=\left(\begin{array}{cc}
\mathrm{D}_{+}^{S \otimes E} & \alpha i^{-} \\
\alpha p^{+} & 0
\end{array}\right) .
$$

By [17, Lemma 7.20] $\widetilde{\mathrm{D}}_{+}^{S \otimes E}(\alpha)$ is invertible for all $\alpha \neq 0$. Define a map $\widetilde{\mathrm{D}^{S} \otimes E}(\alpha): \widetilde{\pi_{*}^{\text {spin }}} E \rightarrow \widetilde{\pi_{*}^{\text {spin }}} E$ by

$$
\widetilde{\mathrm{D}}^{S \otimes E}(\alpha):=\left(\begin{array}{cc}
0 & \left(\widetilde{\mathrm{D}}_{+}^{S \otimes E}(\alpha)\right)^{*} \\
\widetilde{\mathrm{D}}_{+}^{S \otimes E}(\alpha) & 0
\end{array}\right) .
$$

We refer to [17, p.943] for the properties of $\widetilde{\mathrm{D}}^{S \otimes E}(\alpha)$.

The $\mathbb{Z}_{2}$-graded $L^{2}$-metric $g^{\pi_{*}^{\text {sin }} E}$ and the $\mathbb{Z}_{2}$-graded unitary connection $\nabla_{*}^{\pi_{*}^{\text {spin }} E, u}$ on $\pi_{*}^{\text {spin }} E \rightarrow B$ project to a $\mathbb{Z}_{2}$-graded Hermitian metric $g^{L}$ and a $\mathbb{Z}_{2}$-graded unitary connection $\nabla^{L}$ on $L \rightarrow X$ with respect to 2.4 .9 respectively. Define a unitary connection $\widetilde{\nabla^{\pi_{*}^{\text {spin }}}} E, u$ on $\widetilde{\pi_{*}^{\text {spin }}} E \rightarrow B$ by

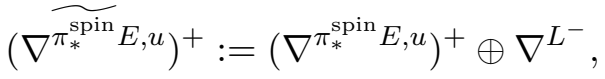

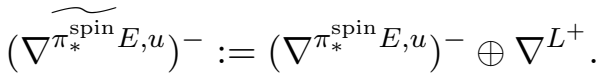

That is, $\widetilde{\nabla_{*}^{\text {spin }} E, u}:=\nabla_{*}^{\pi_{*}^{\text {spin }} E, u} \oplus \nabla^{L, \text { op }}$ as $\mathbb{Z}_{2}$-graded unitary connections. Note that $\widetilde{\nabla_{*}^{\text {spin }}} E, u$ depends on the choice of $L \rightarrow B$ satisfying the MF property with respect to $\mathrm{D}^{S \otimes E}$. However, for the clarity of the notation we will only emphasize the dependence when necessary.

Choose and fix $a \in(0,1)$ and let $\alpha:[0, \infty) \rightarrow[0,1]$ be a smooth function such that $\alpha(t)=0$ for all $t \leq a$ and $\alpha(t)=1$ for all $t \geq 1$. The choice of $a$ is actually immaterial. Define the Bismut superconnection $\widehat{\mathbb{B}}^{E}$ on $\widetilde{\pi_{*}^{\text {spin }}} E \rightarrow B$ by

$$
\widehat{\mathbb{B}}^{E}=\widetilde{\mathrm{D}}^{S \otimes E}(1)+\widetilde{\nabla_{*}^{\text {spin }} E, u}-\widehat{\mathbb{B}}_{[2]}^{E},
$$


where $\widehat{\mathbb{B}}_{[2]}^{E} \in \Omega^{2}\left(B, \operatorname{End}^{-}\left(\widetilde{\pi_{*}^{\text {spin }}} E\right)\right)$ acts on $\Omega\left(B, \pi_{*}^{\text {spin }} E\right)$ by $\frac{c(T)}{4}$, and acts on $\Omega\left(B, L^{\mathrm{op}}\right)$ by zero. For convenience we still use the notation $\frac{c(T)}{4}$ for $\widehat{\mathbb{B}}_{[2]}^{E}$. The rescaled Bismut superconnection $\widehat{\mathbb{B}}_{t}^{E}$ is given by

$$
\widehat{\mathbb{B}}_{t}^{E}=\sqrt{t} \widetilde{\mathrm{D}}^{S \otimes E}(\alpha(t))+\widetilde{\nabla_{*}^{\text {spin }} E, u}-\frac{c(T)}{4 \sqrt{t}} .
$$

Since $\widetilde{\mathrm{D}}^{S \otimes E}(\alpha(t))$ is invertible for $t$ sufficiently large (more precisely, for $t \geq 1)$, we have [17, (7.24)]:

$$
\lim _{t \rightarrow \infty} \operatorname{ch}\left(\widehat{\mathbb{B}}_{t}^{E}\right)=0 .
$$

Remark 4. As noted in [17, p.943], when $t \rightarrow 0$ (more precisely, for $t \leq a$ ) the rescaled Bismut superconnection $\widehat{\mathbb{B}}_{t}^{E}$ decouples, i.e.

$$
\widehat{\mathbb{B}}_{t}^{E}=\left(\sqrt{t} \mathrm{D}^{S \otimes E}+\nabla^{\pi_{*}^{\mathrm{spin}} E, u}-\frac{c(T)}{4 \sqrt{t}}\right) \oplus \nabla^{L, \mathrm{op}}=\mathbb{B}_{t}^{E} \oplus \nabla^{L, \mathrm{op}} .
$$

It follows from Remark 4 that we have [17, (7.23)]:

$$
\lim _{t \rightarrow 0} \operatorname{ch}\left(\widehat{\mathbb{B}}_{t}^{E}\right)=\lim _{t \rightarrow 0} \operatorname{ch}\left(\mathbb{B}_{t}^{E}\right)-\operatorname{ch}\left(\nabla^{L}\right)=\int_{X / B} \widehat{A}\left(\nabla^{T^{V} X}\right) \wedge \operatorname{ch}\left(\nabla^{E}\right)-\operatorname{ch}\left(\nabla^{L}\right) .
$$

The Bismut-Cheeger eta form associated to $\widehat{\mathbb{B}}^{E}[17,(7.25)]$ is defined to be

$$
\widehat{\eta}^{E}\left(g^{E}, \nabla^{E}, T^{H} X, g^{T^{V} X}, L\right)=\int_{0}^{\infty} \operatorname{str}\left(\frac{d \widehat{\mathbb{B}}_{t}^{E}}{d t} e^{-\frac{1}{2 \pi i}\left(\widehat{\mathbb{B}}_{t}^{E}\right)^{2}}\right) d t .
$$

Note that $\widehat{\eta}^{E}\left(g^{E}, \nabla^{E}, T^{H} X, g^{T^{V} X}, L\right)$ does not depend on the choice of $\alpha$.

The local FIT for $\mathrm{D}^{S \otimes E}$ without Assumption 1 is given by the local FIT for $\widetilde{\mathrm{D}}^{S \otimes E}(1)$ [17, (7.26)], i.e.

$$
d \widehat{\eta}^{E}\left(g^{E}, \nabla^{E}, T^{H} X, g^{T^{V} X}, L\right)=\int_{X / B} \widehat{A}\left(\nabla^{T^{V} X}\right) \wedge \operatorname{ch}\left(\nabla^{E}\right)-\operatorname{ch}\left(\nabla^{L}\right) .
$$

Remark 5. The purpose of putting the projected $\mathbb{Z}_{2}$-graded unitary connection $\nabla^{L}$ on $L \rightarrow B$ [17, p.943] is to obtain the local FIT for $\widetilde{\mathrm{D}}^{S \otimes E}(1)$. However, for our purpose we will choose other $\mathbb{Z}_{2}$-graded unitary connection on $L \rightarrow B$. More precisely, let $\widetilde{\nabla}^{L}$ be a $\mathbb{Z}_{2}$-graded unitary connection on $L \rightarrow B$, not necessarily projected from $\nabla_{*}^{\pi_{*}^{\text {spin }} E, u}$. Then the rescaled Bismut superconnection

$$
\widehat{\mathbb{B}}_{t}^{E}:=\sqrt{t} \widetilde{\mathrm{D}}^{S \otimes E}(\alpha(t))+\left(\nabla^{\pi_{*}^{\mathrm{spin}} E, u} \oplus \widetilde{\nabla}^{L, \mathrm{op}}\right)-\frac{c(T)}{4 \sqrt{t}}
$$

still satisfies the analogs of (2.4.11) and 2.4.12). The corresponding BismutCheeger eta form can still be defined, but it depends on $\widetilde{\nabla}^{L}$. The analog of 2.4.13 still holds. Henceforth we write

$$
\widehat{\eta}^{E}\left(g^{E}, \nabla^{E}, T^{H} X, g^{T^{V} X}, L, \widetilde{\nabla}^{L}\right)
$$


for the corresponding Bismut-Cheeger eta form when the unitary connection $\widetilde{\nabla}^{L}$ on $L \rightarrow B$ is not projected from $\pi_{*}^{\text {spin }} E \rightarrow B$.

2.5. The analytic index in differential $K$-theory. In this subsection we review the definition of Freed-Lott differential $K$-theory and the analytic index in differential $K$-theory defined under and without Assumption 1 respectively [17]. The setup of the local FIT in [17] is defined for $\operatorname{spin}^{c}$ fibers, but for our purpose we need spin fibers.

The Freed-Lott differential $K$-group $\widehat{K}_{\mathrm{FL}}(X)$ is the abelian group generated by quadruples $\mathcal{E}=\left(E, g^{E}, \nabla^{E}, \omega\right)$, where $E \rightarrow X$ is a complex vector bundle with a Hermitian metric $g^{E}$ and a unitary connection $\nabla^{E}$, and $\omega \in \frac{\Omega^{\text {odd }}(X)}{\operatorname{Im}(d)}$. The only relation is $\mathcal{E}_{0}=\mathcal{E}_{1}$ if and only if there exists a generator $\left(F, g^{F}, \nabla^{F}, \omega^{F}\right)$ of $\widehat{K}_{\mathrm{FL}}(X)$ such that

$$
\begin{aligned}
& E_{0} \oplus F \cong E_{1} \oplus F \\
& \omega_{0}-\omega_{1}=\operatorname{CS}\left(\nabla^{E_{0}} \oplus \nabla^{F}, \nabla^{E_{1}} \oplus \nabla^{F}\right) \text { in } \frac{\Omega^{\text {odd }}(X)}{\operatorname{Im}(d)} .
\end{aligned}
$$

In $(2.5 .2)$, instead of writing $F^{*}\left(\nabla^{E_{1}} \oplus \nabla^{F}\right)$, where $F: E_{0} \oplus F \rightarrow E_{1} \oplus F$ is a smooth bundle isomorphism given by (2.5.1), we follow the convention in [17] that $F^{*}$ is suppressed ${ }^{1}$ The differential $K$-group $\widehat{K}_{\mathrm{FL}}(X)$ can also be described in terms of $\mathbb{Z}_{2}$-graded generators, i.e. $\mathcal{E}=\left(E, g^{E}, \nabla^{E}, \omega\right)$, where $E \rightarrow X, g^{E}$ and $\nabla^{E}$ are $\mathbb{Z}_{2}$-graded. Two $\mathbb{Z}_{2}$-graded generators $\mathcal{E}_{0}$ and $\mathcal{E}_{1}$ are equal in $\widehat{K}_{\mathrm{FL}}(X)$ if and only if there exist $\mathbb{Z}_{2}$-graded generators $\mathcal{W}=\left(W, g^{W}, \nabla^{W}, \omega_{W}\right)$ and $\mathcal{V}=\left(V, g^{V}, \nabla^{V}, \omega_{V}\right)$ of $\widehat{K}_{\mathrm{FL}}(X)$ of the form

$$
W^{+}=W^{-}, g^{W^{+}}=g^{W^{-}}, \nabla^{W^{+}}=\nabla^{W^{-}},
$$

and similarly for $\mathcal{V}$, such that

$$
\begin{aligned}
& E_{0} \oplus W \cong E_{1} \oplus V \text { as } \mathbb{Z}_{2} \text {-graded complex vector bundles, } \\
& \omega_{0}-\omega_{1}=\operatorname{CS}\left(\nabla^{E_{0}} \oplus \nabla^{W}, \nabla^{E_{1}} \oplus \nabla^{V}\right) \text { in } \frac{\Omega^{\text {odd }}(X)}{\operatorname{Im}(d)}
\end{aligned}
$$

Let $\pi: X \rightarrow B$ be a submersion with closed, oriented and spin fibers of even dimension, and $\mathcal{E}=\left(E, g^{E}, \nabla^{E}, \omega\right)$ a generator of $\widehat{K}_{\mathrm{FL}}(X)$. If Assumption 1 is satisfied, then the differential analytic index $\operatorname{ind}_{\mathrm{FL}}^{\mathrm{a}}(\mathcal{E}) \in \widehat{K}_{\mathrm{FL}}(B)$ [17. Definition 3.12] is defined to be

$$
\begin{aligned}
\operatorname{ind}_{\mathrm{FL}}^{\mathrm{a}}(\mathcal{E})= & \left(\operatorname{ker}\left(\mathrm{D}^{S \otimes E}\right), g^{\operatorname{ker}\left(\mathrm{D}^{S \otimes E}\right)}, \nabla^{\operatorname{ker}\left(\mathrm{D}^{S \otimes E}\right)},\right. \\
& \left.\int_{X / B} \widehat{A}\left(\nabla^{T^{V} X}\right) \wedge \omega+\widetilde{\eta}^{E}\left(g^{E}, \nabla^{E}, T^{H} X, g^{T^{V} X}\right)\right) .
\end{aligned}
$$

\footnotetext{
${ }^{1}$ This convention will also be applied to Corollary 1 and 2
} 
If Assumption 1 is not satisfied, the differential analytic index ind $\mathrm{FL}_{\mathrm{aL}}^{\mathrm{a}}(\mathcal{E} ; L) \in$ $\widehat{K}_{\mathrm{FL}}(B)$ [17, Definition 7.27] is defined to be

$$
\operatorname{ind}_{\mathrm{FL}}^{\mathrm{a}}(\mathcal{E} ; L)=\left(L, g^{L}, \nabla^{L}, \int_{X / B} \widehat{A}\left(\nabla^{T^{V} X}\right) \wedge \omega+\widehat{\eta}^{E}\left(g^{E}, \nabla^{E}, T^{H} X, g^{T^{V} X}, L\right)\right) \text {, }
$$

where $L \rightarrow B$ is a fixed choice of $\mathbb{Z}_{2}$-graded complex vector bundle satisfying the MF property with respect to $\mathrm{D}^{S \otimes E}$, and $g^{L}, \nabla^{L}$ are the projected $\mathbb{Z}_{2^{-}}$ graded Hermitian metric and projected $\mathbb{Z}_{2}$-graded unitary connection on $L \rightarrow B$.

\section{MAin Results}

In this section we prove the main results of this paper. Henceforth by geometric data we mean the quadruple

$$
\left(g^{E}, \nabla^{E}, T^{H} X, g^{T^{V} X}\right)
$$

as defined in Section 2.3 .

\subsection{A variational formula of the Bismut-Cheeger eta form without} the kernel bundle assumption and its applications. In this subsection we prove a variational formula of the Bismut-Cheeger eta form without Assumption 1 (Proposition 1).

The following lemma, which is an immediate consequence of [6, Theorem 2.10], roughly says the Bismut-Cheeger eta form defined without Assumption 1 is stable under perturbation of split quadruple.

Lemma 1. Let $\pi: X \rightarrow B$ be a submersion with closed, oriented and spin fibers of even dimension and $E \rightarrow X$ a complex vector bundle. Denote by $\left(g^{E}, \nabla^{E}, T^{H} X, g^{T^{V}} X\right)$ a fixed choice of geometric data. Let $L \rightarrow B$ be a $\mathbb{Z}_{2^{-}}$ graded complex vector bundle satisfying the MF property with respect to $\mathrm{D}^{S \otimes E}$. Denote by $g^{L}$ the projected Hermitian metric and $\nabla^{L}$ the projected $\mathbb{Z}_{2}$-graded unitary connection on $L \rightarrow B$. Let $\alpha:(0, \infty) \rightarrow[0,1]$ be the smooth function given in Section 2.4 and $\widehat{\mathbb{B}}^{E}$ the Bismut superconnection on $\widetilde{\pi_{*}^{\text {spin }}} E \rightarrow B$ defined by 2.4 .10$)$. Let $\left(W, g^{W}, \nabla^{W}, s_{W}\right)$ be a split quadruple over $B$ (cf. Section 2.1). Define a superconnection $\mathbb{A}^{W}$ on $W \rightarrow B$ by

$$
\mathbb{A}^{W}=s_{W}+\nabla^{W}
$$

and define the rescaled superconnection $\mathbb{A}_{t}^{W}$ by

$$
\mathbb{A}_{t}^{W}=\sqrt{t} \alpha(t) s_{W}+\nabla^{W} .
$$

If $\widehat{\mathbb{B}}^{E, W}$ is the Bismut superconnection on $\widetilde{\pi_{*}^{\text {spin }}} E \oplus W \rightarrow B$ defined by

$$
\widehat{\mathbb{B}}^{E, W}=\widehat{\mathbb{B}}^{E} \oplus \mathbb{A}^{W},
$$

then

$\widehat{\eta}^{E, W}\left(g^{E}, \nabla^{E}, T^{H} X, g^{T^{V} X}, L \oplus W, \nabla^{L} \oplus \nabla^{W}\right)=\widehat{\eta}^{E}\left(g^{E}, \nabla^{E}, T^{H} X, g^{T^{V} X}, L\right)$ 
in $\frac{\Omega^{\text {odd }}(B)}{\operatorname{Im}(d)}$, where the left-hand side of 3.1 .3 denotes the Bismut-Cheeger eta form associated to $\widehat{\mathbb{B}}^{E, W}$.

Proof. Note that for all $t \in(0, \infty)$ we have $\widehat{\mathbb{B}}_{t}^{E, W}=\widehat{\mathbb{B}}_{t}^{E} \oplus \mathbb{A}_{t}^{W}$. By 2.1.12, for any $t<T \in(0, \infty)$ we have

$$
\begin{aligned}
\operatorname{CS}\left(\widehat{\mathbb{B}}_{T}^{E, W}, \widehat{\mathbb{B}}_{t}^{E, W}\right) & =\operatorname{CS}\left(\widehat{\mathbb{B}}_{T}^{E} \oplus \mathbb{A}_{T}^{W}, \widehat{\mathbb{B}}_{t}^{E} \oplus \mathbb{A}_{t}^{W}\right) \\
& =\operatorname{CS}\left(\widehat{\mathbb{B}}_{T}^{E}, \widehat{\mathbb{B}}_{t}^{E}\right)+\operatorname{CS}\left(\mathbb{A}_{T}^{W}, \mathbb{A}_{t}^{W}\right)
\end{aligned}
$$

in $\frac{\Omega^{\text {odd }}(B)}{\operatorname{Im}(d)}$. Denote by $\widetilde{\eta}^{W}$ the Bismut-Cheeger eta form associated to $\mathbb{A}^{W}$.

By letting $T \rightarrow \infty$ and $t \rightarrow 0$ in above we have

$$
\begin{aligned}
& \widehat{\eta}^{E, W}\left(g^{E}, \nabla^{E}, T^{H} X, g^{T^{V} X}, L \oplus W, \nabla^{L} \oplus \nabla^{W}\right) \\
= & \widehat{\eta}^{E}\left(g^{E}, \nabla^{E}, T^{H} X, g^{T^{V} X}, L\right)+\widetilde{\eta}^{W} .
\end{aligned}
$$

Thus proving 3.1 .3 is equivalent to proving $\widetilde{\eta}^{W}=0 \in \frac{\Omega^{\text {odd }}(B)}{\operatorname{Im}(d)}$. Since the quadruple $\left(W, g^{W}, \nabla^{W}, s_{W}\right)$ splits, it follows from [6, (2.23)] that

$$
\left(\mathbb{A}_{t}^{W}\right)^{2}=t \alpha(t)^{2} P^{\operatorname{Im}\left(s_{W}\right)}+\left(\nabla^{W}\right)^{2}
$$

where $P^{\operatorname{Im}\left(s_{W}\right)}: W \rightarrow \operatorname{Im}\left(s_{W}\right)$ is the projection onto $\operatorname{Im}\left(s_{W}\right) \rightarrow B$ with respect to the direct sum decomposition $W=\operatorname{ker}\left(s_{W}\right) \oplus \operatorname{Im}\left(s_{W}\right)$. By [6, (2.24)] we have

$$
\operatorname{str}\left(\frac{d \mathbb{A}_{t}^{W}}{d t} e^{-\frac{1}{2 \pi i}\left(\mathbb{A}_{t}^{W}\right)^{2}}\right)=0 .
$$

Thus $\widetilde{\eta}^{W}=0 \in \Omega^{\text {odd }}(B)$, and therefore 3.1 .3 holds.

Recall from [4, p.289] that a $\mathbb{Z}_{2}$-graded complex vector bundle $E \rightarrow X$ defines an element in $K(X)$ by $E \mapsto\left[E^{+}\right]-\left[E^{-}\right]$. Two $\mathbb{Z}_{2}$-graded complex vector bundles $E \rightarrow X$ and $F \rightarrow X$ define the same element in $K(X)$ if and only if there exist two complex vector bundles $G \rightarrow X$ and $H \rightarrow X$ such that

$$
\begin{aligned}
& E^{+} \oplus G \cong F^{+} \oplus H, \\
& E^{-} \oplus G \cong F^{-} \oplus H .
\end{aligned}
$$

We now prove a variational formula of the Bismut-Cheeger eta form without Assumption 1. The proof is actually similar to the ones of [20, 24]. The extra technicality is caused by different choices of $\mathbb{Z}_{2}$-graded complex vector bundle satisfying the MF property with respect to $\mathrm{D}^{S \otimes E}$.

Proposition 1. Let $\pi: X \rightarrow B$ be a submersion with closed, oriented and spin fibers of even dimension $n$, and $E \rightarrow X$ a complex vector bundle. Fix $k \in\{0,1\}$. Denote by $\left(g_{k}^{E}, \nabla_{k}^{E}, T_{k}^{H} X, g_{k}^{T^{V} X}\right)$ the geometric data, $\mathrm{D}_{k}^{S \otimes E}$ the corresponding twisted spin Dirac operator, and $L_{k} \rightarrow B$ a $\mathbb{Z}_{2}$-graded complex vector bundle satisfying the $\mathrm{MF}$ property with respect to $\mathrm{D}_{k}^{S \otimes E}$. 
Denote by $g^{L_{k}}$ and $\nabla^{L_{k}}$ the $\mathbb{Z}_{2}$-graded Hermitian metric and the $\mathbb{Z}_{2}$-graded unitary connection on $L_{k} \rightarrow B$ projected from $\pi_{*}^{\text {spin }} E \rightarrow B$. Then for $k \in$ $\{0,1\}$, there exist a $\mathbb{Z}_{2}$-graded complex vector bundle $W_{k} \rightarrow B$ of the form $W_{k}^{+}=W_{k}^{-}$with a $\mathbb{Z}_{2}$-graded Hermitian metric $g^{W_{k}}$ of the form $g^{W_{k}^{+}}=g^{W_{k}^{-}}$ and a $\mathbb{Z}_{2}$-graded unitary connection $\nabla^{W_{k}}$ of the form $\nabla^{W_{k}^{+}}=\nabla^{W_{k}^{-}}$such that

$$
L_{0} \oplus W_{0} \cong L_{1} \oplus W_{1}
$$

as $\mathbb{Z}_{2}$-graded complex vector bundles via a smooth $\mathbb{Z}_{2}$-graded bundle isomorphism $h: L_{1} \oplus W_{1} \rightarrow L_{0} \oplus W_{0}$, and

$$
\begin{aligned}
& \widehat{\eta}^{E}\left(g_{1}^{E}, \nabla_{1}^{E}, T_{1}^{H} X, g_{1}^{T^{V} X}, L_{1}\right)-\widehat{\eta}^{E}\left(g_{0}^{E}, \nabla_{0}^{E}, T_{0}^{H} X, g_{0}^{T^{V} X}, L_{0}\right) \\
= & \int_{X / B} \widetilde{\widehat{A}}\left(\nabla_{0}^{T^{V} X}, \nabla_{1}^{T^{V} X}\right) \wedge \operatorname{ch}\left(\nabla_{0}^{E}\right)+\int_{X / B} \widehat{A}\left(\nabla_{1}^{T^{V} X}\right) \wedge \operatorname{CS}\left(\nabla_{0}^{E}, \nabla_{1}^{E}\right) \\
& \quad-\operatorname{CS}\left(h^{*}\left(\nabla^{L_{0}} \oplus \nabla^{W_{0}}\right), \nabla^{L_{1}} \oplus \nabla^{W_{1}}\right)
\end{aligned}
$$

in $\frac{\Omega^{\text {odd }}(B)}{\operatorname{Im}(d)}$, where $\widetilde{A}\left(\nabla_{0}^{T^{V} X}, \nabla_{1}^{T^{V} X}\right)$ is defined by 2.1 .15 .

Proof. Since the space of the splitting map is affine, there exists a smooth path of horizontal distributions $\left\{T_{t}^{H} X \rightarrow X\right\}_{t \in[0,1]}$ joining $T_{0}^{H} X \rightarrow X$ and $T_{1}^{H} X \rightarrow X$. By Section 2.1 and above, there exists a smooth path

$$
\left(g_{t}^{E}, \nabla_{t}^{E}, T_{t}^{H} X, g_{t}^{T^{V} X}\right) \text { with } t \in[0,1],
$$

joining $\left(g_{0}^{E}, \nabla_{0}^{E}, T_{0}^{H} X, g_{0}^{T^{V} X}\right)$ and $\left(g_{1}^{E}, \nabla_{1}^{E}, T_{1}^{H} X, g_{1}^{T^{V} X}\right)$. From (3.1.6) one can define a new path, denoted by $\alpha$, joining $\left(g_{0}^{E}, \nabla_{0}^{E}, T_{0}^{H} X, g_{0}^{T^{V} X}\right)$ and $\left(g_{1}^{E}, \nabla_{1}^{E}, T_{1}^{H} X, g_{1}^{T^{V} X}\right)$, by

$$
\alpha(t)=\left\{\begin{array}{ll}
\left(g_{0}^{E}, \nabla_{0}^{E}, T_{2 t}^{H} X, g_{2 t}^{T^{V}} X\right), & \text { for } t \in\left[0, \frac{1}{2}\right], \\
\left(g_{2 t-1}^{E}, \nabla_{2 t-1}^{E}, T_{1}^{H} X, g_{1}^{T^{V} X}\right), & \text { for } t \in\left[\frac{1}{2}, 1\right]
\end{array} .\right.
$$

Here, for $t \in\left[0, \frac{1}{2}\right]$, the path $\left(T_{2 t}^{H} X, g_{2 t}^{T^{V}} X\right)$ joining $\left(T_{0}^{H} X, g_{0}^{T^{V}} X\right)$ and $\left(T_{1}^{H} X, g_{t}^{T^{V}} X\right)$ is induced by $(3.1 .6)$; for $t \in\left[\frac{1}{2}, 1\right]$, the path $\left(g_{2 t-1}^{E}, \nabla_{2 t-1}^{E}\right)$ joining $\left(g_{0}^{E}, \nabla_{0}^{E}\right)$ and $\left(g_{1}^{E}, \nabla_{1}^{E}\right)$ is induced by 3.1 .6 .

Consider the following diagram

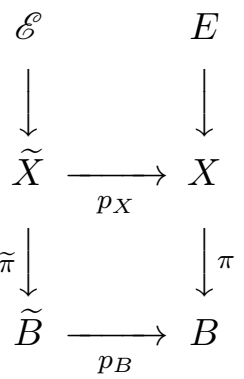


where $\mathscr{E}:=p_{X}^{*} E$. The smooth path $(3.1 .7)$ defines the geometric data

$$
\left(g^{\mathscr{E}}, \nabla^{\mathscr{E}}, T^{H} \widetilde{X}, g^{T^{V}} \tilde{X}\right)
$$

where $\nabla^{\mathscr{E}}$ is defined by 2.1.6. Since the fibers of $\pi: X \rightarrow B$ are oriented and spin, the same is true for the fibers of $\widetilde{\pi}: \widetilde{X} \rightarrow \widetilde{B}$.

Denote by $\mathrm{D}^{S \otimes \mathscr{E}}: \widetilde{\pi}_{*}^{\text {spin }} \mathscr{E} \rightarrow \widetilde{\pi}_{*}^{\text {spin } \mathscr{E}}$ the twisted spin Dirac operator defined by the geometric data (3.1.8). Since Assumption 1 is not satisfied, we choose and fix a $\mathbb{Z}_{2}$-graded complex vector bundle $\mathcal{L} \rightarrow \widetilde{B}$ satisfying the MF property with respect to $\mathrm{D}^{S \otimes \mathscr{E}}$. Denote by $\mathcal{K} \rightarrow \widetilde{B}$ a $\mathbb{Z}_{2}$-graded complementary subbundle of $\widetilde{\pi}_{*}^{\text {spin }} \mathscr{E} \rightarrow \widetilde{B}$, i.e.

$$
\left(\widetilde{\pi}_{*}^{\text {spin }} \mathscr{E}\right)^{ \pm}=\mathcal{K}^{ \pm} \oplus \mathcal{L}^{ \pm}
$$

Denote by $g^{\mathcal{L}}$ the projected $\mathbb{Z}_{2}$-graded Hermitian metric on $\mathcal{L} \rightarrow \widetilde{B}$.

As in $(2.1 .2)$ we have the following smooth bundle isomorphisms

$$
\begin{aligned}
i_{B, 0}^{*}\left(\widetilde{\pi}_{*}^{\text {spin }} \mathscr{E}\right)^{ \pm} & \cong i_{B, 1}^{*}\left(\widetilde{\pi}_{*}^{\text {spin }} \mathscr{E}\right)^{ \pm} \\
i_{B, 0}^{*} \mathcal{K}^{ \pm} & \cong i_{B, 1}^{*} \mathcal{K}^{ \pm} \\
i_{B, 0}^{*} \mathcal{L}^{ \pm} & \cong i_{B, 1}^{*} \mathcal{L}^{ \pm}
\end{aligned}
$$

Write $K^{ \pm} \rightarrow B$ for $i_{B, 0}^{*} \mathcal{K}^{ \pm} \rightarrow B$ and $L^{ \pm} \rightarrow B$ for $i_{B, 0}^{*} \mathcal{L}^{ \pm} \rightarrow B$. Also, write $g^{L_{k}}=i_{B, k}^{*} g^{\mathcal{L}}$ for $k \in\{0,1\}$. On the other hand, since

$$
i_{B, k}^{*}\left(\widetilde{\pi}_{*}^{\text {spin }} \mathscr{E}\right)^{ \pm} \cong\left(\pi_{*}^{\text {spin }} E\right)^{ \pm}
$$

for $k=0$ and $k=1$ respectively, it follows that $\pi_{*}^{\text {spin }} E \rightarrow B$ admits a direct sum decomposition, given by

$$
\left(\pi_{*}^{\text {spin }} E\right)^{+}=K^{+} \oplus L^{+}, \quad\left(\pi_{*}^{\text {spin }} E\right)^{-}=K^{-} \oplus L^{-} .
$$

Since

$$
\left.\mathrm{D}^{S \otimes \mathscr{E}}\right|_{i_{B, k}^{*} \widetilde{\pi}_{*}^{\operatorname{spin} \mathscr{E}}}=\mathrm{D}_{k}^{S \otimes E},
$$

it follows that $\mathrm{D}_{k}^{S \otimes E}:\left(\pi_{*}^{\text {spin }} E\right)^{+} \rightarrow\left(\pi_{*}^{\text {spin }} E\right)^{-}$is block diagonal with respect to 3.1.11 and the restriction $\mathrm{D}_{k}^{S \otimes E}: K^{+} \rightarrow K^{-}$is an isomorphism. Thus $L \rightarrow B$ satisfies the MF property with respect to $\mathrm{D}_{k}^{S \otimes E}$ for $k=0$ and $k=1$ respectively. Therefore the $K$-theoretic analytic index of $[E] \in K(X)$ is given by

$$
\operatorname{ind}^{\mathrm{a}}([E])=\left[L^{+}\right]-\left[L^{-}\right] .
$$

By assumption, the $K$-theoretic analytic index of $[E] \in K(X)$ are given by

$$
\operatorname{ind}^{\mathrm{a}}([E])=\left[L_{0}^{+}\right]-\left[L_{0}^{-}\right] \text {and } \operatorname{ind}^{\mathrm{a}}([E])=\left[L_{1}^{+}\right]-\left[L_{1}^{-}\right]
$$

respectively. Since the $K$-theoretic analytic index is well defined, it follows from $(3.1 .3)$ that there exist complex vector bundles $G_{k} \rightarrow B$ and $H_{k} \rightarrow B$, 
where $k \in\{0,1\}$, such that

$$
\begin{aligned}
& L_{0}^{+} \oplus G_{0} \cong L^{+} \oplus H_{0}, \\
& L_{0}^{-} \oplus G_{0} \cong L^{-} \oplus H_{0}, \\
& L_{1}^{+} \oplus G_{1} \cong L^{+} \oplus H_{1}, \\
& L_{1}^{-} \oplus G_{1} \cong L^{-} \oplus H_{1} .
\end{aligned}
$$

It follows from $(3.1 .13)$ that

$$
\begin{aligned}
& L_{0}^{+} \oplus\left(G_{0} \oplus H_{1}\right) \cong L^{+} \oplus H_{0} \oplus H_{1} \cong L^{+} \oplus H_{1} \oplus H_{0} \cong L_{1}^{+} \oplus\left(G_{1} \oplus H_{0}\right), \\
& L_{0}^{-} \oplus\left(G_{0} \oplus H_{1}\right) \cong L^{-} \oplus H_{0} \oplus H_{1} \cong L^{-} \oplus H_{1} \oplus H_{0} \cong L_{1}^{-} \oplus\left(G_{1} \oplus H_{0}\right) .
\end{aligned}
$$

Write

$$
\begin{aligned}
H^{+} & =H^{-}=H_{0} \oplus H_{1}, \\
W_{0}^{+} & =W_{0}^{-}=G_{0} \oplus H_{1}, \\
W_{1}^{+} & =W_{1}^{-}=G_{1} \oplus H_{0}
\end{aligned}
$$

respectively. Define $\mathbb{Z}_{2}$-graded complex vector bundles $H \rightarrow B, W_{0} \rightarrow B$ and $W_{1} \rightarrow B$ by

$$
H=H^{+} \oplus H^{-}, \quad W_{0}=W_{0}^{+} \oplus W_{0}^{-}, \quad W_{1}=W_{1}^{+} \oplus W_{1}^{-} .
$$

Write $\mathcal{H}=p_{B}^{*} H$. By (3.1.14) we have the following commutative diagram

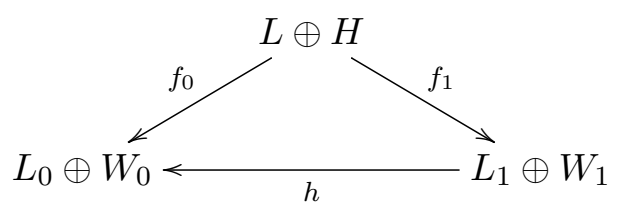

where $f_{0}$ and $f_{1}$ are the resulting smooth $\mathbb{Z}_{2}$-graded bundle isomorphisms, and $h:=f_{0} \circ f_{1}^{-1}$.

For $k \in\{0,1\}$, put a Hermitian metric $g^{W_{k}^{+}}$on $W_{k}^{+} \rightarrow B$ and a unitary connection $\nabla^{W_{k}^{+}}$on $W_{k}^{+} \rightarrow B$ with respect to $g^{W_{k}^{+}}$. Then define a $\mathbb{Z}_{2}$-graded Hermitian metric $g^{W_{k}}$ and a $\mathbb{Z}_{2}$-graded unitary connection $\nabla^{W_{k}}$ on $W_{k} \rightarrow B$ by $g^{W_{k}^{-}}:=g^{W_{k}^{+}}$and $\nabla^{W_{k}^{-}}:=\nabla^{W_{k}^{+}}$.

Note that $g_{0}^{L \oplus H}:=f_{0}^{*}\left(g^{L_{0}} \oplus g^{W_{0}}\right)$ and $g_{1}^{L \oplus H}:=f_{1}^{*}\left(g^{L_{1}} \oplus g^{W_{1}}\right)$ are $\mathbb{Z}_{2^{-}}$ graded Hermitian metrics on $L \oplus H \rightarrow B$, and

$$
\nabla_{0}:=f_{0}^{*}\left(\nabla^{L_{0}} \oplus \nabla^{W_{0}}\right), \quad \nabla_{1}:=f_{1}^{*}\left(\nabla^{L_{1}} \oplus \nabla^{W_{1}}\right)
$$

are $\mathbb{Z}_{2}$-graded connections on $L \oplus H \rightarrow B$ that are unitary with respect to $g_{0}^{L \oplus H}$ and $g_{1}^{L \oplus H}$ respectively. Define a $\mathbb{Z}_{2}$-graded unitary connection $\widetilde{\nabla} \mathcal{L} \oplus \mathcal{H}$ on $\mathcal{L} \oplus \mathcal{H} \rightarrow \widetilde{B}$ by 2.1.6 such that

$$
i_{B, 0}^{*} \widetilde{\nabla}^{\mathcal{L} \oplus \mathcal{H}}=\nabla_{0}, \quad i_{B, 1}^{*} \widetilde{\nabla}^{\mathcal{L} \oplus \mathcal{H}}=\nabla_{1} .
$$


The rescaled Bismut superconnection $\widehat{\mathbb{B}}_{t}^{\mathscr{E}, \mathcal{H}}$ on the $\mathbb{Z}_{2}$-graded infinite rank bundle $\widetilde{\pi}_{*}^{\text {spin }} \mathscr{E} \oplus \mathcal{L}^{\text {op }} \oplus \mathcal{H}^{\text {op }} \rightarrow \widetilde{B}$ is defined to be

$$
\widehat{\mathbb{B}}_{t}^{\mathscr{E}, \mathcal{H}}=\widehat{\mathbb{B}}_{[0], t}^{\mathscr{E}, \mathcal{H}}+\left(\nabla^{\widetilde{\pi}_{*}^{\text {sin }} \mathscr{E}, u} \oplus \widetilde{\nabla}^{\mathcal{L} \oplus \mathcal{H}, \mathrm{op}}\right)-\widehat{\mathbb{B}}_{[2], t}^{\mathscr{E}, \mathcal{H}},
$$

where

$$
\widehat{\mathbb{B}}_{[0], t}^{\mathscr{E}, \mathcal{H}}=\sqrt{t}\left(\begin{array}{cccc}
0 & 0 & \left(\widetilde{\mathrm{D}}_{+}^{S \otimes} \mathscr{E}^{\mathscr{E}}(\alpha(t))\right)^{*} & 0 \\
0 & 0 & 0 & \alpha(t) \mathrm{id} \\
\widetilde{\mathrm{D}}_{+}^{S \otimes \mathscr{E}}(\alpha(t)) & 0 & 0 & 0 \\
0 & \alpha(t) \text { id } & 0 & 0
\end{array}\right)
$$

and $\widehat{\mathbb{B}}_{[2], t}^{\mathscr{E}, \mathcal{H}} \in \Omega^{2}\left(\widetilde{B}\right.$, End $\left.^{-}\left(\widetilde{\pi}_{*}^{\text {spin }} \mathscr{E} \oplus \mathcal{L}^{\text {op }} \oplus \mathcal{H}^{\text {op }}\right)\right)$ acts on $\Omega\left(\widetilde{B}, \widetilde{\pi}_{*}^{\text {spin }} \mathscr{E}\right)$ by $\frac{c(\widetilde{T})}{4 \sqrt{t}}$ and acts on $\Omega\left(\widetilde{B}, \mathcal{L}^{\text {op }} \oplus \mathcal{H}^{\text {op }}\right)$ by zero. Here $\widetilde{T}$ is the curvature 2 -form of the fiber bundle $\widetilde{\pi}: \widetilde{X} \rightarrow \widetilde{B}$. For convenience we still use the notation $\frac{c(\widetilde{T})}{4 \sqrt{t}}$ for $\widehat{\mathbb{B}}_{[2], t}^{\mathscr{E}, \mathcal{H}}$. Thus

$$
\widehat{\mathbb{B}}_{t}^{\mathscr{E}, \mathcal{H}}=\widehat{\mathbb{B}}_{[0], t}^{\mathscr{E}, \mathcal{H}}+\left(\nabla^{\widetilde{\pi}_{*}^{\text {sin }} \mathscr{E}, u} \oplus \widetilde{\nabla}^{\mathcal{L} \oplus \mathcal{H}, \mathrm{op}}\right)-\frac{c(\widetilde{T})}{4 \sqrt{t}}
$$

The (unrescaled) Bismut superconnection $\widehat{\mathbb{B}}^{\mathscr{E}, \mathcal{H}}$ is recovered by taking $t=1$ in $\widehat{\mathbb{B}}_{t}^{\mathscr{E}, \mathcal{H}}$.

By Remark 4, for $t \leq a$ we have

$$
\widehat{\mathbb{B}}_{t}^{\mathscr{E}, \mathcal{H}}=\mathbb{B}_{t}^{\mathscr{E}} \oplus \widetilde{\nabla}^{\mathcal{L} \oplus \mathcal{H}, \text { op }} .
$$

Thus by Remark 5 the analog of $(2.4 .12)$ holds, i.e.

$$
\begin{aligned}
\lim _{t \rightarrow 0} \operatorname{ch}\left(\widehat{\mathbb{B}}_{t}^{\mathscr{E}, \mathcal{H}}\right) & =\lim _{t \rightarrow 0} \operatorname{ch}\left(\mathbb{B}_{t}^{\mathscr{E}}\right)-\operatorname{ch}\left(\nabla^{\mathcal{L} \oplus \mathcal{H}}\right) \\
& =\int_{\widetilde{X} / \widetilde{B}} \widehat{A}\left(\nabla^{T^{V} \widetilde{X}}\right) \wedge \operatorname{ch}\left(\nabla^{\mathscr{E}}\right)-\operatorname{ch}\left(\widetilde{\nabla}^{\mathcal{L} \oplus \mathcal{H}}\right) .
\end{aligned}
$$

On the other hand, since $\widehat{\mathbb{B}}_{[0], t}^{\mathscr{E}, \mathcal{H}}$ is invertible for $t \geq 1$, it follows that the analog of 2.4.11 holds, i.e.

$$
\lim _{t \rightarrow \infty} \operatorname{ch}\left(\widehat{\mathbb{B}}_{t}^{\mathscr{E}, \mathcal{H}}\right)=0 .
$$

Define the Bismut-Cheeger eta form associated to $\widehat{\mathbb{B}}^{\mathscr{E}, \mathcal{H}}$ by $\widehat{\eta}^{\mathscr{E}, \mathcal{H}}\left(g^{\mathscr{E}}, \nabla^{\mathscr{E}}, T^{H} \widetilde{X}, g^{T^{V} \widetilde{X}}, \mathcal{L} \oplus \mathcal{H}, \widetilde{\nabla}^{\mathcal{L} \oplus \mathcal{H}}\right)=\int_{0}^{\infty} \operatorname{str}\left(\frac{d \widehat{\mathbb{B}}_{t}^{\mathscr{E}, \mathcal{H}}}{d t} e^{-\frac{1}{2 \pi i}\left(\widehat{\mathbb{B}}_{t}^{\mathscr{E}, \mathcal{H}}\right)^{2}}\right) d t$.

We now temporarily suppress the data defining the Bismut-Cheeger eta form to shorten the expression. By (3.1.17), 3.1.18) and Remark 5 we have

$$
d \widehat{\eta}^{\mathscr{E}, \mathcal{H}}=\int_{\widetilde{X} / \widetilde{B}} \widehat{A}\left(\nabla^{T^{V} \widetilde{X}}\right) \wedge \operatorname{ch}\left(\nabla^{\mathscr{E}}\right)-\operatorname{ch}\left(\widetilde{\nabla}^{\mathcal{L} \oplus \mathcal{H}}\right) .
$$


Denote by $i: \partial \widetilde{B} \rightarrow \widetilde{B}$ the inclusion map. By taking $M \rightarrow B$ to be $\widetilde{B} \rightarrow B$ and $\omega$ to be $\widehat{\eta}^{\mathscr{E}, \mathcal{H}}$ in 2.1.4 we have

$$
-\left(i_{B, 1}^{*} \widehat{\eta}^{\mathscr{E}, \mathcal{H}}-i_{B, 0}^{*} \widehat{\eta}^{\mathscr{E}, \mathcal{H}}\right)=-\int_{\partial \widetilde{B} / B} i^{*} \widehat{\eta}^{\mathscr{E}, \mathcal{H}}=\int_{\widetilde{B} / B} d^{\widetilde{B}} \widehat{\eta}^{\mathscr{E}, \mathcal{H}}-d^{B} \int_{\widetilde{B} / B} \widehat{\eta}^{\mathscr{E}, \mathcal{H}}
$$

By modding out exact forms in 3.1 .20 , it follows from 2.1.3) and 3.1 .19 that

$$
\begin{aligned}
& i_{B, 1}^{*} \widehat{\eta}^{\mathscr{E}, \mathcal{H}}-i_{B, 0}^{*} \widehat{\eta}^{\mathscr{E}, \mathcal{H}}=-\int_{\widetilde{B} / B} d^{\widetilde{B}} \widehat{\eta}^{\mathscr{E}, \mathcal{H}} \\
= & -\int_{\widetilde{B} / B}\left(\int_{\widetilde{X} / \widetilde{B}} \widehat{A}\left(\nabla^{T^{V} \widetilde{X}}\right) \wedge \operatorname{ch}\left(\nabla^{\mathscr{E}}\right)-\operatorname{ch}\left(\widetilde{\nabla^{\mathcal{L}} \oplus \mathcal{H}}\right)\right) \\
= & \int_{\widetilde{B} / B} \int_{\widetilde{X} / \widetilde{B}}\left(-\widehat{A}\left(\nabla^{T^{V} \widetilde{X}}\right) \wedge \operatorname{ch}\left(\nabla^{\mathscr{E}}\right)\right)-\operatorname{CS}\left(\nabla_{0}, \nabla_{1}\right)
\end{aligned}
$$

in $\frac{\Omega^{\text {odd }}(B)}{\operatorname{Im}(d)}$. Since $f_{1}$ covers the identity $\operatorname{map~id}_{B}$, it follows that

$$
\begin{aligned}
\operatorname{CS}\left(\nabla_{0}, \nabla_{1}\right) & =\operatorname{CS}\left(f_{0}^{*}\left(\nabla^{L_{0}} \oplus \nabla^{W_{0}}\right), f_{1}^{*}\left(\nabla^{L_{1}} \oplus \nabla^{W_{1}}\right)\right) \\
& =\operatorname{CS}\left(\left(f_{1}^{-1}\right)^{*} f_{0}^{*}\left(\nabla^{L_{0}} \oplus \nabla^{W_{0}}\right), \nabla^{L_{1}} \oplus \nabla^{W_{1}}\right)
\end{aligned}
$$

Note that $h^{*}=\left(f_{1}^{-1}\right)^{*} \circ f_{0}^{*}$. Since $\int_{X / B} \circ \int_{\widetilde{X} / X}=\int_{\widetilde{X} / B}=\int_{\widetilde{B} / B} \circ \int_{\widetilde{X} / \widetilde{B}}$, it follows that

$$
\int_{\widetilde{B} / B} \int_{\widetilde{X} / \widetilde{B}}\left(-\widehat{A}\left(\nabla^{T^{V} \widetilde{X}}\right) \wedge \operatorname{ch}\left(\nabla^{\mathscr{E}}\right)\right)=\int_{X / B} \int_{\widetilde{X} / X}\left(-\widehat{A}\left(\nabla^{T^{V} \widetilde{X}}\right) \wedge \operatorname{ch}\left(\nabla^{\mathscr{E}}\right)\right) .
$$

By (3.1.8), 2.1.3 and 2.1.15) we have

$$
\begin{aligned}
& \int_{\widetilde{X} / X}\left(-\widehat{A}\left(\nabla^{T^{V} \tilde{X}}\right) \wedge \operatorname{ch}\left(\nabla^{\mathscr{E}}\right)\right)= \widetilde{\widehat{A}} \\
&\left(\nabla_{0}^{T^{V} X}, \nabla_{1}^{T^{V} X}\right) \wedge \operatorname{ch}\left(\nabla_{0}^{E}\right) \\
&+\widehat{A}\left(\nabla_{1}^{T^{V} X}\right) \wedge \operatorname{CS}\left(\nabla_{0}^{E}, \nabla_{1}^{E}\right) .
\end{aligned}
$$

By putting (3.1.22) and 3.1.23) into 3.1.21) we obtain

$$
\begin{aligned}
& i_{B, 1}^{*} \widehat{\eta}^{\mathscr{E}, \mathcal{H}}-i_{B, 0}^{*} \widehat{\eta}^{\mathscr{E}, \mathcal{H}} \\
= & \int_{X / B} \widetilde{\widehat{A}}\left(\nabla_{0}^{T^{V} X}, \nabla_{1}^{T^{V} X}\right) \wedge \operatorname{ch}\left(\nabla_{0}^{E}\right)+\int_{X / B} \widehat{A}\left(\nabla_{1}^{T^{V} X}\right) \wedge \operatorname{CS}\left(\nabla_{0}^{E}, \nabla_{1}^{E}\right) \\
& \quad-\operatorname{CS}\left(h^{*}\left(\nabla^{L_{0}} \oplus \nabla^{W_{0}}\right), \nabla^{L_{1}} \oplus \nabla^{W_{1}}\right)
\end{aligned}
$$


in $\frac{\Omega^{\text {odd }}(B)}{\operatorname{Im}(d)}$. On the other hand, it follows from $3.1 .12,3.1 .15$ and 3.1 .16 that

$$
\begin{aligned}
& i_{B, 1}^{*} \widehat{\eta}^{\mathscr{E}, \mathcal{H}}\left(g^{\mathscr{E}}, \nabla^{\mathscr{E}}, T^{H} \tilde{X}, g^{T^{V}} \widetilde{X}, \mathcal{L} \oplus \mathcal{H}, \widetilde{\nabla}^{\mathcal{L} \oplus \mathcal{H}}\right) \\
= & \widehat{\eta}^{E, W}\left(g_{1}^{E}, \nabla_{1}^{E}, T_{1}^{H} X, g_{1}^{T^{V} X}, L_{1} \oplus W, \nabla^{L_{1}} \oplus \nabla^{W_{1}}\right) .
\end{aligned}
$$

Define $s_{W_{1}} \in \Gamma\left(B, \operatorname{End}^{-}\left(W_{1}\right)\right)$ by $s_{W_{1}}=\left(\begin{array}{cc}0 & \text { id } \\ \text { id } & 0\end{array}\right)$. By Example 1 the quadruple $\left(W_{1}, g^{W_{1}}, \nabla^{W_{1}}, s_{W_{1}}\right)$ over $B$ splits. Define a superconnection $\mathbb{A}^{W_{1}}$ on $W_{1} \rightarrow B$ by 3.1.1 and its rescaled superconnection $\mathbb{A}_{t}^{W_{1}}$ by 3.1 .2 . The Bismut superconnection $\widehat{\mathbb{B}}^{E, W_{1}}$ defining the Bismut-Cheeger eta form $\widehat{\eta}^{E, W_{1}}\left(g_{1}^{E}, \nabla_{1}^{E}, T_{1}^{H} X, g_{1}^{T^{V} X}, L_{1} \oplus W_{1}, \nabla^{L_{1}} \oplus \nabla^{W_{1}}\right)$ is given by

$$
\widehat{\mathbb{B}}^{E, W_{1}}=\widehat{\mathbb{B}}^{E} \oplus \mathbb{A}^{W_{1}} \text {, }
$$

It follows from Lemma 1 that $(3.1 .25)$ becomes

$$
\begin{aligned}
& i_{B, 1}^{*} \widehat{\eta}^{\mathscr{E}, \mathcal{H}}\left(g^{\mathscr{E}}, \nabla^{\mathscr{E}}, T^{H} \tilde{X}, g^{T^{V} \tilde{X}}, \mathcal{L} \oplus \mathcal{H}, \widetilde{\nabla}^{\mathcal{L} \oplus \mathcal{H}}\right) \\
= & \widehat{\eta}^{E}\left(g_{1}^{E}, \nabla_{1}^{E}, T_{1}^{H} X, g_{1}^{T^{V} X}, L_{1}\right)
\end{aligned}
$$

in $\frac{\Omega^{\text {odd }}(B)}{\operatorname{Im}(d)}$. By considering the split quadruple $\left(W_{0}, g^{W_{0}}, \nabla^{W_{0}}, s_{W_{0}}\right)$ defined in a similar way as above we have

$$
\begin{aligned}
& i_{B, 0}^{*} \widehat{\eta}^{\mathscr{E}, \mathcal{H}}\left(g^{\mathscr{E}}, \nabla^{\mathscr{E}}, T^{H} \widetilde{X}, g^{T^{V}} \tilde{X}, \mathcal{L} \oplus \mathcal{H}, \widetilde{\nabla}^{\mathcal{L} \oplus \mathcal{H}}\right) \\
= & \widehat{\eta}^{E}\left(g_{0}^{E}, \nabla_{0}^{E}, T_{0}^{H} X, g_{0}^{T^{V} X}, L_{0}\right)
\end{aligned}
$$

in $\frac{\Omega^{\text {odd }}(B)}{\operatorname{Im}(d)}$. Thus by putting 3.1.26 and 3.1.27 into 3.1.24 we have

$$
\begin{aligned}
& \widehat{\eta}^{E}\left(g_{1}^{E}, \nabla_{1}^{E}, T_{1}^{H} X, g_{1}^{T^{V} X}, L_{1}\right)-\widehat{\eta}^{E}\left(g_{0}^{E}, \nabla_{0}^{E}, T_{0}^{H} X, g_{0}^{T^{V} X}, L_{0}\right) \\
= & \int_{X / B} \widetilde{\widehat{A}}\left(\nabla_{0}^{T^{V} X}, \nabla_{1}^{T^{V} X}\right) \wedge \operatorname{ch}\left(\nabla_{0}^{E}\right)+\int_{X / B} \widehat{A}\left(\nabla_{1}^{T^{V} X}\right) \wedge \operatorname{CS}\left(\nabla_{0}^{E}, \nabla_{1}^{E}\right) \\
& -\operatorname{CS}\left(h^{*}\left(\nabla^{L_{0}} \oplus \nabla^{W_{0}}\right), \nabla^{L_{1}} \oplus \nabla^{W_{1}}\right)
\end{aligned}
$$

in $\frac{\Omega^{\text {odd }}(B)}{\operatorname{Im}(d)}$. Thus 3.1 .5 holds.

We now give another proofs of [17, (3) and (4) of Corollary 7.36].

Corollary 1. Let $\pi: X \rightarrow B$ be a submersion with closed, oriented and spin fibers of even dimension, and $\mathcal{E}=\left(E, g^{E}, \nabla^{E}, \omega\right)$ a generator of $\widehat{K}_{\mathrm{FL}}(X)$. If $L_{0} \rightarrow B$ and $L_{1} \rightarrow B$ are $\mathbb{Z}_{2}$-graded complex vector bundles satisfying the MF property with respect to $\mathrm{D}^{S \otimes E}$, then

$$
\operatorname{ind}_{\mathrm{FL}}^{\mathrm{a}}\left(\mathcal{E} ; L_{0}\right)=\operatorname{ind}_{\mathrm{FL}}^{\mathrm{a}}\left(\mathcal{E} ; L_{1}\right) .
$$


Proof. By (2.5.4), 2.5.5) and (2.5.7), proving (3.1.28) is equivalent to showing the existence of $\mathbb{Z}_{2}$-graded generators $\mathcal{W}_{0}$ and $\mathcal{W}_{1}$ of $\widehat{K}_{\mathrm{FL}}(B)$ of the form 2.5.3 such that

$$
L_{0} \oplus W_{0} \cong L_{1} \oplus W_{1}
$$

as $\mathbb{Z}_{2}$-graded complex vector bundles and

$$
\begin{aligned}
& \widehat{\eta}^{E}\left(g^{E}, \nabla^{E}, T^{H} X, g^{T^{V} X}, L_{1}\right)-\widehat{\eta}^{E}\left(g^{E}, \nabla^{E}, T^{H} X, g^{T^{V} X}, L_{0}\right) \\
= & \operatorname{CS}\left(\nabla^{L_{1}} \oplus \nabla^{W_{1}}, \nabla^{L_{0}} \oplus \nabla^{W_{0}}\right)
\end{aligned}
$$

in $\frac{\Omega^{\text {odd }}(B)}{\operatorname{Im}(d)}$. First note that 3.1 .29 follows from 3.1 .15 . By taking $g_{1}^{E}=$ $g_{0}^{E}=g^{E}, \nabla_{1}^{E}=\nabla_{0}^{E}=\nabla^{E}, T_{1}^{H} X=T_{2}^{H} X=T^{H} X$ and $g_{1}^{T^{V} X}=g_{0}^{T^{V} X}=$ $g^{T^{V} X}$ in 3.1.5, we obtain 3.1.30 by 2.1.8.

Corollary 2. Let $\pi: X \rightarrow B$ be a submersion with closed, oriented and spin fibers of even dimension, and $\mathcal{E}=\left(E, g^{E}, \nabla^{E}, \omega\right)$ a generator of $\widehat{K}_{\mathrm{FL}}(X)$. If Assumption 1 is satisfied, then for any $\mathbb{Z}_{2}$-graded complex vector bundle $L \rightarrow B$ satisfying the MF property with respect to $\mathrm{D}^{S \otimes E}$ we have

$$
\operatorname{ind}_{\mathrm{FL}}^{\mathrm{a}}(\mathcal{E} ; L)=\operatorname{ind}_{\mathrm{FL}}^{\mathrm{a}}(\mathcal{E}),
$$

where the right-hand side of (3.1.31) is given by (2.5.6).

Proof. Since the kernel bundle $\operatorname{ker}\left(\mathrm{D}^{S \otimes E}\right) \rightarrow B$ exists, and it satisfies the MF property with respect to $\mathrm{D}^{S \otimes E}$, it follows from Corollary 1 that $\operatorname{ind}_{\mathrm{FL}}^{\mathrm{a}}(\mathcal{E} ; L)=$ $\operatorname{ind}_{\mathrm{FL}}^{\mathrm{a}}\left(\mathcal{E} ; \operatorname{ker}\left(\mathrm{D}^{S \otimes E}\right)\right)$. Thus proving 3.1 .31 is equivalent to proving

$$
\operatorname{ind}_{\mathrm{FL}}^{\mathrm{a}}\left(\mathcal{E} ; \operatorname{ker}\left(\mathrm{D}^{S \otimes E}\right)\right)=\operatorname{ind}_{\mathrm{FL}}^{\mathrm{a}}(\mathcal{E}) .
$$

As in the proof of Corollary 2, proving $(3.1 .32)$ is equivalent to showing the existence of $\mathbb{Z}_{2}$-graded generators $\mathcal{W}_{0}$ and $\mathcal{W}_{1}$ of $\widehat{K}_{\mathrm{FL}}(B)$ of the form 2.5.3 such that

$$
\operatorname{ker}\left(\mathrm{D}^{S \otimes E}\right) \oplus W_{1} \cong \operatorname{ker}\left(\mathrm{D}^{S \otimes E}\right) \oplus W_{0}
$$

as $\mathbb{Z}_{2}$-graded complex vector bundles and

$$
\begin{aligned}
& \widehat{\eta}^{E}\left(g^{E}, \nabla^{E}, T^{H} X, g^{T^{V} X}, \operatorname{ker}\left(\mathrm{D}^{S \otimes E}\right)\right)-\widetilde{\eta}^{E}\left(g^{E}, \nabla^{E}, T^{H} X, g^{T^{V} X}\right) \\
= & \operatorname{CS}\left(\nabla^{\operatorname{ker}\left(\mathrm{D}^{S \otimes E}\right)} \oplus \nabla^{W_{1}}, \nabla^{\operatorname{ker}\left(\mathrm{D}^{S \otimes E}\right)} \oplus \nabla^{W_{0}}\right)
\end{aligned}
$$

in $\frac{\Omega^{\text {odd }}(B)}{\operatorname{Im}(d)}$. We choose $W_{0} \rightarrow B$ and $W_{1} \rightarrow B$ to be zero $\mathbb{Z}_{2}$-graded complex vector bundles, so that 3.1.33 is satisfied. Since

$\mathrm{CS}\left(\nabla^{\operatorname{ker}\left(\mathrm{D}^{S \otimes E}\right)} \oplus \nabla^{W_{1}}, \nabla^{\operatorname{ker}\left(\mathrm{D}^{S \otimes E}\right)} \oplus \nabla^{W_{0}}\right)=\mathrm{CS}\left(\nabla^{\operatorname{ker}\left(\mathrm{D}^{S \otimes E}\right)}, \nabla^{\operatorname{ker}\left(\mathrm{D}^{S \otimes E}\right)}\right)=0$ in $\frac{\Omega^{\text {odd }}(B)}{\operatorname{Im}(d)}$, it follows that 3.1 .34 becomes

$$
\widehat{\eta}^{E}\left(g^{E}, \nabla^{E}, T^{H} X, g^{T^{V} X}, \operatorname{ker}\left(\mathrm{D}^{S \otimes E}\right)\right)=\widetilde{\eta}^{E}\left(g^{E}, \nabla^{E}, T^{H} X, g^{T^{V} X}\right)
$$


in $\frac{\Omega^{\text {odd }}(B)}{\operatorname{Im}(d)}$. First note that in the current situation, the $\mathbb{Z}_{2}$-graded Hermitian bundle $L^{\mathrm{op}} \rightarrow B$ in the definition of $\widehat{\mathbb{B}}^{E}$ given by $\left(2.4 .10\right.$ is $\operatorname{ker}\left(\mathrm{D}^{S \otimes E}\right)^{\text {op }} \rightarrow$

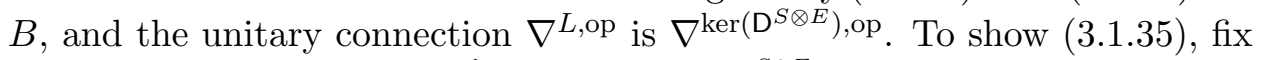

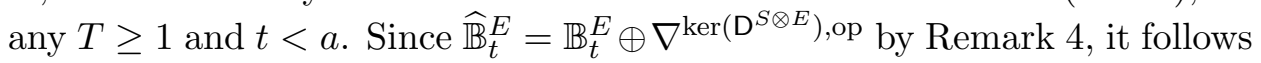
from (2.1.13), 2.1.11) and (2.1.12) that

$$
\begin{aligned}
& \operatorname{CS}\left(\widehat{\mathbb{B}}_{T}^{E}, \widehat{\mathbb{B}}_{t}^{E}\right)-\operatorname{CS}\left(\mathbb{B}_{T}^{E}, \mathbb{B}_{t}^{E}\right) \\
= & \operatorname{CS}\left(\widehat{\mathbb{B}}_{T}^{E}, \widehat{\mathbb{B}}_{t}^{E}\right)-\operatorname{CS}\left(\mathbb{B}_{T}^{E}, \mathbb{B}_{t}^{E}\right)-\operatorname{CS}\left(\nabla^{\operatorname{ker}\left(\mathrm{D}^{S \otimes E}\right), \mathrm{op}}, \nabla^{\operatorname{ker}\left(\mathrm{D}^{S \otimes E}\right), \mathrm{op}}\right) \\
= & \operatorname{CS}\left(\widehat{\mathbb{B}}_{T}^{E}, \widehat{\mathbb{B}}_{t}^{E}\right)-\operatorname{CS}\left(\mathbb{B}_{T}^{E} \oplus \nabla^{\operatorname{ker}\left(\mathrm{D}^{S \otimes E}\right), \mathrm{op}}, \mathbb{B}_{t}^{E} \oplus \nabla^{\operatorname{ker}\left(\mathrm{D}^{S \otimes E}\right), \mathrm{op}}\right) \\
= & \operatorname{CS}\left(\widehat{\mathbb{B}}_{T}^{E}, \widehat{\mathbb{B}}_{t}^{E}\right)+\operatorname{CS}\left(\mathbb{B}_{t}^{E} \oplus \nabla^{\operatorname{ker}\left(\mathrm{D}^{S \otimes E}\right), \mathrm{op}}, \mathbb{B}_{T}^{E} \oplus \nabla^{\operatorname{ker}\left(\mathrm{D}^{S \otimes E}\right), \mathrm{op}}\right) \\
= & \operatorname{CS}\left(\widehat{\mathbb{B}}_{T}^{E}, \mathbb{B}_{T}^{E} \oplus \nabla^{\operatorname{ker}\left(\mathrm{D}^{S \otimes E}\right), \mathrm{op}}\right)
\end{aligned}
$$

in $\frac{\Omega^{\text {odd }}(B)}{\operatorname{Im}(d)}$. By letting $T \rightarrow \infty$ and $t \rightarrow 0$ in above, we have

$$
\begin{aligned}
& \widehat{\eta}^{E}\left(g^{E}, \nabla^{E}, T^{H} X, g^{T^{V} X}, \operatorname{ker}\left(\mathrm{D}^{S \otimes E}\right)\right)-\widetilde{\eta}^{E}\left(g^{E}, \nabla^{E}, T^{H} X, g^{T^{V} X}\right) \\
= & \lim _{T \rightarrow \infty} \operatorname{CS}\left(\widehat{\mathbb{B}}_{T}^{E}, \mathbb{B}_{T}^{E} \oplus \nabla^{\operatorname{ker}\left(\mathrm{D}^{S \otimes E}\right), \text { op }}\right)
\end{aligned}
$$

in $\frac{\Omega^{\text {odd }}(B)}{\operatorname{Im}(d)}$. By the estimates in $\left.[4, \S 9.3]\right]^{2}$ we have

$$
\lim _{T \rightarrow \infty} \operatorname{CS}\left(\widehat{\mathbb{B}}_{T}^{E}, \mathbb{B}_{T}^{E} \oplus \nabla^{\mathrm{ker}\left(\mathrm{D}^{S \otimes E}\right), \mathrm{op}}\right)=0 .
$$

Thus 3.1.35 holds, and therefore so does 3.1.32.

3.2. Some properties of Cheeger-Chern-Simons class. In this subsection we prove some properties of the Cheeger-Chern-Simons class that are needed to prove Theorem 1. These properties roughly say that the Cheeger-Chern-Simons classes of a complex flat vector bundle with flat connection with respect to different gradings are equal.

Lemma 2. Let $\left(E, v, \nabla^{E}\right)$ be a flat $\mathbb{Z}$-graded cochain complex of the form 2.2.8. If $\operatorname{rank}\left(E^{+}\right)=\operatorname{rank}\left(E^{-}\right)$, then

$$
\sum_{k=0}^{m}(-1)^{k} \operatorname{CCS}\left(E^{k}, \nabla^{k}\right)=\operatorname{CCS}\left(E, \nabla^{E}\right),
$$

where the left-hand side of (3.2.1) is the Cheeger-Chern-Simons class of $\left(E, \nabla^{E}\right)$ with respect to the $\mathbb{Z}$ grading given by $(2.2 .9)$ and the right-hand side of 3.2 .1 is the Cheeger-Chern-Simons class of $\left(E, \nabla^{E}\right)$ with respect to the $\mathbb{Z}_{2}$ grading given by 2.2 .10 .

\footnotetext{
${ }^{2}$ This argument is inspired by the proof of [18, Proposition 9].
} 
Proof. Consider $E \rightarrow X$ as a $\mathbb{Z}$-graded complex flat vector bundle with $\mathbb{Z}$ graded flat connection $\nabla^{E}$. Since $\left(\nabla^{k}\right)^{2}=0$ for each $0 \leq k \leq m$, there exists $\ell_{k} \in \mathbb{N}$ such that $\ell_{k} E^{k} \cong \ell_{k} \mathbb{C}^{r_{k}}$, where $r_{k}=\operatorname{rank}\left(E^{k}\right)$. By (2.2.1) a differential form representative of $\operatorname{CCS}\left(E^{k}, \nabla^{k}\right)$ is given by

$$
\frac{1}{\ell_{k}} \operatorname{CS}\left(\nabla_{0}^{\ell_{k} E^{k}}, \ell_{k} \nabla^{k}\right)
$$

where $\nabla_{0}^{\ell_{k} E^{k}}$ is a trivial connection on $\ell_{k} E^{k} \rightarrow X$. Let $\ell$ be the least common multiple of $\ell_{0}, \ldots, \ell_{m}$. Then there exist unique $d_{0}, \ldots, d_{m} \in \mathbb{N}$ such that $\ell=d_{k} \ell_{k}$ for each $0 \leq k \leq m$. Since

$$
\ell E^{k}=d_{k} \ell_{k} E^{k} \cong d_{k} \ell_{k} \mathbb{C}^{r_{k}}=\ell \mathbb{C}^{r_{k}}
$$

it follows from 2.1 .9 and 2.1 .8 that

$$
\begin{aligned}
\frac{1}{\ell_{k}} \operatorname{CS}\left(\nabla_{0}^{\ell_{k} E^{k}}, \ell_{k} \nabla^{k}\right) & =\frac{1}{\ell} \operatorname{CS}\left(d_{k} \nabla_{0}^{\ell_{k} E^{k}}, d_{k} \ell_{k} \nabla^{k}\right) \\
& =\frac{1}{\ell} \operatorname{CS}\left(d_{k} \nabla_{0}^{\ell_{k} E^{k}}, \nabla_{0}^{\ell E^{k}}\right)+\frac{1}{\ell} \operatorname{CS}\left(\nabla_{0}^{\ell E^{k}}, \ell \nabla^{k}\right),
\end{aligned}
$$

where $\nabla_{0}^{\ell E^{k}}$ is a trivial connection on $\ell E^{k} \rightarrow X$. By Remark 2 we have

$$
\operatorname{CS}\left(d_{k} \nabla_{0}^{\ell_{k} E^{k}}, \nabla_{0}^{\ell E^{k}}\right)=\operatorname{ch}^{\text {odd }}\left(g_{k}\right) \in \Omega_{\mathbb{Q}}^{\text {odd }}(X ; \mathbb{C})
$$

for some smooth map $g_{k}: X \rightarrow \mathrm{GL}\left(\ell r_{k} ; \mathbb{C}\right)$. By (3.2.3) and (3.2.4) we have

$$
\sum_{k=0}^{m} \frac{(-1)^{k}}{\ell_{k}} \operatorname{CS}\left(\nabla_{0}^{\ell_{k} E^{k}}, \ell_{k} \nabla^{k}\right)=\sum_{k=0}^{m} \frac{(-1)^{k}}{\ell} \operatorname{CS}\left(\nabla_{0}^{\ell E^{k}}, \ell \nabla^{k}\right)+\sum_{k=0}^{m} \frac{(-1)^{k}}{\ell} \operatorname{ch}^{\text {odd }}\left(g_{k}\right) .
$$

Recall that the connections $\nabla^{ \pm}$on $E^{ \pm} \rightarrow X$ are defined by 2.2.8). Note that

$$
\nabla_{0}^{\ell E^{+}}:=\bigoplus_{k} \nabla_{0}^{\ell E^{2 k}}, \quad \nabla_{0}^{\ell E^{-}}:=\bigoplus_{k} \nabla_{0}^{\ell E^{2 k+1}}
$$

are trivial connections on $\ell E^{ \pm} \rightarrow X$ respectively. By 2.1 .9 the first term of the right-hand side of 3.2 .5 becomes

$$
\sum_{k=0}^{m} \frac{(-1)^{k}}{\ell} \operatorname{CS}\left(\nabla_{0}^{\ell E^{k}}, \ell \nabla^{k}\right)=\frac{1}{\ell} \operatorname{CS}\left(\nabla_{0}^{\ell E^{+}}, \ell \nabla^{+}\right)-\frac{1}{\ell} \operatorname{CS}\left(\nabla_{0}^{\ell E^{-}}, \ell \nabla^{-}\right)
$$


Since $\operatorname{rank}\left(E^{+}\right)=\operatorname{rank}\left(E^{-}\right),(3.2 .2)$ induces an isomorphism $j: \ell E^{+} \rightarrow$ $\ell E^{-}$. Since $j^{*} \nabla_{0}^{\ell E^{-}}=\nabla_{0}^{\ell E^{+}}$, by $(2.1 .8)$ we have

$$
\begin{aligned}
& \frac{1}{\ell} \operatorname{CS}\left(\nabla_{0}^{\ell E^{+}}, \ell \nabla^{+}\right) \\
= & \frac{1}{\ell} \operatorname{CS}\left(\nabla_{0}^{\ell E^{+}}, j^{*} \ell \nabla^{-}\right)+\frac{1}{\ell} \operatorname{CS}\left(j^{*} \ell \nabla^{-}, \ell \nabla^{+}\right) \\
= & \frac{1}{\ell} \operatorname{CS}\left(\nabla_{0}^{\ell E^{+}}, j^{*} \ell \nabla^{-}\right)+\frac{1}{\ell} \operatorname{CS}\left(j^{*} \ell \nabla^{-}, j^{*} \nabla_{0}^{\ell E^{-}}\right)+\frac{1}{\ell} \operatorname{CS}\left(j^{*} \nabla_{0}^{\ell E^{-}}, \nabla_{0}^{\ell E^{+}}\right) \\
= & \frac{1}{\ell} \operatorname{CS}\left(\nabla_{0}^{\ell E^{+}}, j^{*} \ell \nabla^{-}\right)+\frac{1}{\ell} \operatorname{CS}\left(j^{*} \ell \nabla^{-}, j^{*} \nabla_{0}^{\ell E^{-}}\right) .
\end{aligned}
$$

By (3.2.6) and (3.2.7), (3.2.5) becomes

$$
\sum_{k=0}^{m} \frac{(-1)^{k}}{\ell_{k}} \operatorname{CS}\left(\nabla_{0}^{\ell_{k} E^{k}}, \ell_{k} \nabla^{k}\right)=\frac{1}{\ell} \operatorname{CS}\left(j^{*} \ell \nabla^{-}, \ell \nabla^{+}\right)+\sum_{k=0}^{m} \frac{(-1)^{k}}{\ell} \operatorname{ch}^{\text {odd }}\left(g_{k}\right) \text {. }
$$

Since the $\bmod \mathbb{Q}$ reduction of the de Rham class of the left-hand side of (3.2.8) is the left-hand side of (3.2.1), and the same is true for the righthand side of (3.2.8) and (3.2.1), it follows that (3.2.1) holds.

The following lemma follows from (2.1.10) and (2.1.14).

Lemma 3. Let $F^{ \pm} \rightarrow X$ be a complex flat vector bundle with flat connection $\nabla^{ \pm}$. Define $F:=F^{+} \oplus F^{-}$and $\nabla^{F}:=\nabla^{+} \oplus \nabla^{-}$. If $F \rightarrow X$ and $\nabla^{F}$ are ungraded direct sums, then

$$
\operatorname{CCS}\left(F, \nabla^{F}\right)=\operatorname{CCS}\left(F^{+}, \nabla^{+}\right)+\operatorname{CCS}\left(F^{-}, \nabla^{-}\right) .
$$

If $\operatorname{rank}\left(F^{+}\right)=\operatorname{rank}\left(F^{-}\right)$and $F \rightarrow X, \nabla^{F}$ are $\mathbb{Z}_{2}$-graded direct sums, then

$$
\operatorname{CCS}\left(F, \nabla^{F}\right)=\operatorname{CCS}\left(F^{+}, \nabla^{+}\right)-\operatorname{CCS}\left(F^{-}, \nabla^{-}\right) .
$$

Note that 3.2 .10$)$ is not a consequence of Lemma 2 since the $\mathbb{Z}_{2}$ grading of $\left(F, \nabla^{F}\right)$ does not come from a $\mathbb{Z}$-graded flat cochain complex.

\subsection{The real part of the Riemann-Roch-Grothendieck theorem} for complex flat vector bundles. In this subsection we first prove a $\mathbb{Z}_{2}$-graded version of 1.0 .2 for $\operatorname{dim}(Z)$ even at the differential form level (Theorem 1). Then we apply Theorem 1 and a result by Bismut [7, Theorem $3.12]$ to deduce 11.0 .2 for $\operatorname{dim}(Z)$ even.

Let $n \in \mathbb{N}$. In the proof of Theorem 1 we will use the following notations. Write $g^{n}$ for a trivial metric on the trivial bundle $\mathbb{C}^{n} \rightarrow B$. Let $d^{n}$ be a trivial unitary connection on $\mathbb{C}^{n} \rightarrow B$. Write $\mathbb{C}^{n} \rightarrow B$ for the $\mathbb{Z}_{2}$-graded trivial bundle $\underline{\mathbb{C}^{n}}:=\mathbb{C}^{n} \oplus \mathbb{C}^{n}$ and $g^{n}$ for the $\mathbb{Z}_{2}$-graded trivial metric defined by

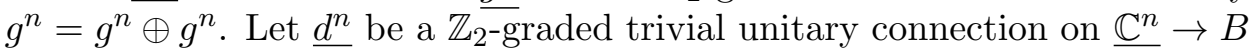
defined by $\underline{d^{n}}=d^{n} \oplus d^{n}$. 
Theorem 1. Let $\pi: X \rightarrow B$ be a submersion with closed fibers $Z$ with $\operatorname{dim}(Z)$ even and $F \rightarrow X$ a $\mathbb{Z}_{2}$-graded complex flat vector bundle of virtual rank zero with $\mathbb{Z}_{2}$-graded flat connection $\nabla^{F}=\nabla^{+} \oplus \nabla^{-}$. Put a $\mathbb{Z}_{2}$-graded Hermitian metric $g^{F}=g^{+} \oplus g^{-}$on $F \rightarrow X$, and the induced Hermitian metric $g^{H\left(Z,\left.F^{ \pm}\right|_{Z}\right)}$ on $H\left(Z,\left.F^{ \pm}\right|_{Z}\right) \rightarrow B$. Define a unitary connection $\nabla^{ \pm, u}$ on $F^{ \pm} \rightarrow X$ with respect to $g^{ \pm}$by $(2.2 .3)$ and a $\mathbb{Z}_{2}$-graded unitary connection $\nabla^{H\left(Z,\left.F^{ \pm}\right|_{Z}\right), u}$ on $H\left(Z,\left.F^{ \pm}\right|_{Z}\right) \rightarrow B$ with respect to $g^{H\left(Z,\left.F^{ \pm}\right|_{Z}\right)}$ by 2.3.7 respectively. Then there exist $k, N, M \in \mathbb{N}$, a smooth isometric isomorphism $\widetilde{j}: k F^{+} \rightarrow k F^{-}$, and a smooth $\mathbb{Z}_{2}$-graded isometric isomorphism $\tilde{f}: k H\left(Z,\left.F^{+}\right|_{Z}\right) \oplus \underline{\mathbb{C}^{M}} \rightarrow k H\left(Z,\left.F^{-}\right|_{Z}\right) \oplus \underline{\mathbb{C}^{N}}$ such that

$$
\mathrm{CS}\left(\widetilde{f}^{*}\left(k \nabla^{H\left(Z, F^{-} \mid z\right), u} \oplus \underline{d^{N}}\right), k \nabla^{H\left(Z, F^{+} \mid z\right), u} \oplus \underline{d^{M}}\right)=\int_{X / B} e\left(\nabla^{T^{V} X}\right) \wedge \operatorname{CS}\left(\widetilde{j}^{*} k \nabla^{-, u}, k \nabla^{+, u}\right)
$$

in $\frac{\Omega^{\text {odd }}(B)}{\Omega_{\mathbb{Q}}^{\text {odd }}(B)}$.

Proof. Let $F \rightarrow X$ be a $\mathbb{Z}_{2}$-graded complex flat vector bundle of virtual rank zero with $\mathbb{Z}_{2}$-graded flat connection $\nabla^{F}=\nabla^{+} \oplus \nabla^{-}$. Then there exist $k_{1} \in \mathbb{N}$ and a smooth bundle isomorphism $j: k_{1} F^{+} \rightarrow k_{1} F^{-}$.

Let $\bullet \in\{$ even, odd $\}$. Define $n_{ \pm}^{\bullet}=\operatorname{rank}\left(H^{\bullet}\left(Z,\left.F^{ \pm}\right|_{Z}\right)\right)$. Since $H^{\bullet}\left(Z,\left.F^{ \pm}\right|_{Z}\right) \rightarrow$ $B$ is a complex flat vector bundle with flat connection $\left.\left.\nabla^{H^{\bullet}\left(Z, F^{ \pm}\right.}\right|_{Z}\right)$, there exist $\ell_{ \pm}^{\bullet} \in \mathbb{N}$ and smooth bundle isomorphisms $h_{ \pm}^{\bullet}: \ell_{ \pm}^{\bullet} H^{\bullet}\left(Z,\left.F^{ \pm}\right|_{Z}\right) \rightarrow \ell_{ \pm}^{\bullet} \mathbb{C}_{ \pm}^{n_{ \pm}^{\bullet}}$. Let $k \in \mathbb{N}$ be the least common multiple of

$$
k_{1}, \quad \ell_{+}^{\text {even }}, \quad \ell_{-}^{\text {even }}, \quad \ell_{+}^{\text {odd }} \text { and } \ell_{-}^{\text {odd }} .
$$

We still denote by

$$
j: k F^{+} \rightarrow k F^{-} \text {and } h_{ \pm}^{\bullet}: k H^{\bullet}\left(Z,\left.F^{ \pm}\right|_{Z}\right) \rightarrow k \mathbb{C}^{n_{ \pm}^{\bullet}}
$$

the resulting smooth bundle isomorphisms. Note that our choice of $k$ guarantees that smooth bundle isomorphisms in $(3.3 .3)$ hold simultaneously.

Put a $\mathbb{Z}_{2}$-graded Hermitian metric $g^{F}=g^{+} \oplus g^{-}$on $F \rightarrow X$. Define a unitary connection $\nabla^{ \pm, u}$ on $F^{ \pm} \rightarrow X$ by 2.2.3 with respect to $g^{ \pm}$. By 2.2.11 there exists $f \in \operatorname{Aut}\left(k F^{+}\right)$such that $k g^{+}=f^{*} j^{*} k g^{-}$. Write $\widetilde{j}=$ $j \circ f$. Thus $\widetilde{j}: k F^{+} \rightarrow k F^{-}$is a smooth isometric isomorphism. On the other hand, since $\widetilde{j}^{*} k \nabla^{-}$is a flat connection on $k F^{+} \rightarrow X$, it follows that

$$
\widetilde{j}:\left(k F^{+}, \widetilde{j}^{*} k \nabla^{-}\right) \rightarrow\left(k F^{-}, k \nabla^{-}\right)
$$

is an isomorphism of complex flat vector bundles ${ }^{3}$ Write $\mathrm{F}=k F^{+}$and $g^{\mathrm{F}}=k g^{+}$. Let $\nabla_{t}^{\mathrm{F}}$ be a smooth curve of connections on $\mathrm{F} \rightarrow X$ such that

$$
\nabla_{1}^{\mathrm{F}}=k \nabla^{+}, \quad \nabla_{0}^{\mathrm{F}}=\widetilde{j}^{*} k \nabla^{-} .
$$

\footnotetext{
${ }^{3}$ We will use the different flat structures $\left(k F^{+}, k \nabla^{+}\right)$and $\left(k F^{+}, \widetilde{j}^{*} k \nabla^{-}\right)$separately.
} 
Consider the pullback of $\mathrm{F} \rightarrow X$ by $p_{X}$ :

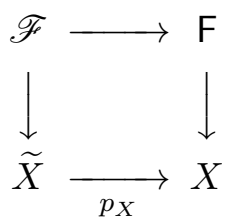

where $\mathscr{F}:=p_{X}^{*}$ F. Define a connection $\nabla^{\mathscr{F}}$ on $\mathscr{F} \rightarrow \widetilde{X}$ by

$$
\nabla^{\mathscr{F}}=\nabla_{t}^{\mathrm{F}}+d t \wedge \frac{\partial}{\partial t}
$$

Note that $\nabla^{\mathscr{F}}$ is not necessarily flat. Define a Hermitian metric on $\mathscr{F} \rightarrow \widetilde{X}$ by $g^{\mathscr{F}}=p_{X}^{*} g^{\mathrm{F}}$, and the unitary connection $\nabla^{\mathscr{F}, u}$ on $\mathscr{F} \rightarrow \widetilde{X}$ by 2.2 .3 . Note that $j^{*} k \nabla^{-, u}$ is a unitary connection on $\mathrm{F} \rightarrow X$ with respect to $g^{\mathrm{F}}$. Since

$$
\nabla_{1}^{\mathrm{F}, u}=\nabla_{1}^{\mathrm{F}}+\frac{1}{2}\left(g^{\mathrm{F}}\right)^{-1}\left(\nabla_{1}^{\mathrm{F}} g^{\mathrm{F}}\right)=k \nabla^{+}+\frac{1}{2}\left(k g^{+}\right)^{-1}\left(k \nabla^{+}\left(k g^{+}\right)\right)=k \nabla^{+, u}
$$

and

$$
\begin{aligned}
\nabla_{0}^{\mathrm{F}, u} & =\nabla_{0}^{\mathrm{F}}+\frac{1}{2}\left(g^{\mathrm{F}}\right)^{-1}\left(\nabla_{0}^{\mathrm{F}} g^{\mathrm{F}}\right) \\
& =\widetilde{j}^{*} k \nabla^{-}+\frac{1}{2}\left(k g^{+}\right)^{-1}\left(\widetilde{j}^{*} k \nabla^{-}\left(k g^{+}\right)\right) \\
& =\widetilde{j}^{*}\left(k \nabla^{-}+\frac{1}{2}\left(k g^{-}\right)^{-1}\left(k \nabla^{-}\left(k g^{-}\right)\right)\right) \\
& =\widetilde{j}^{*} k \nabla^{-, u},
\end{aligned}
$$

it follows that

$$
i_{X, 1}^{*} \nabla^{\mathscr{F}, u}=\nabla_{1}^{\mathrm{F}, u}=k \nabla^{+, u}, \quad i_{X, 0}^{*} \nabla^{\mathscr{F}, u}=\nabla_{0}^{\mathrm{F}, u}=\widetilde{j}^{*} k \nabla^{-, u} .
$$

Temporarily assume that the fibers $Z$ are oriented and spin. Then the fibers of $\widetilde{\pi}: \widetilde{X} \rightarrow \widetilde{B}$, denoted by $\widetilde{Z}$, are also oriented and spin. The geometric data on $\widetilde{\pi}: \widetilde{X} \rightarrow \widetilde{B}$ is given by $\left(g^{\mathscr{F}}, \nabla^{\mathscr{F}}, u, T^{H} \widetilde{X}, g^{T^{V}} \widetilde{X}\right)$, where $T^{H} \widetilde{X} \rightarrow$ $\widetilde{X}$ and $g^{T^{V} \tilde{X}}$ are obtained by pulling back a fixed choice of $T^{H} X \rightarrow X$ and $g^{T^{V} X}$ respectively. The infinite rank $\mathbb{Z}_{2}$-graded complex vector bundle $\widetilde{\pi}_{*}^{\text {spin }}\left(S\left(T^{V} \widetilde{X}\right)^{*} \otimes \mathscr{F}\right) \rightarrow \widetilde{B}$ defined by 2.4 .2 is equipped with a $\mathbb{Z}_{2}$-graded $L^{2}$-metric and a $\mathbb{Z}_{2}$-graded unitary connection $\nabla_{*}^{\widetilde{\pi}_{*}^{\text {spin }}\left(S\left(T^{V} \tilde{X}\right)^{*} \otimes \mathscr{F}\right), u}$ defined by 2.4.3). Define the spin Dirac operator $\mathrm{D}^{S \widehat{\otimes}\left(S^{*} \otimes \mathscr{F}\right)}$ twisted by $S\left(T^{V} \widetilde{X}\right)^{*} \otimes$ $\mathscr{F} \rightarrow X$ by 2.4 .1 , and define $\widetilde{V}$ by 2.3.9). The perturbed twisted spin Dirac operator $\mathrm{D}^{S \otimes}\left(S^{*} \otimes \mathscr{F}\right)+\widetilde{V}$ acting on

$$
\Gamma\left(\tilde{X}, S\left(T^{V} \tilde{X}\right) \widehat{\otimes}\left(S\left(T^{V} \tilde{X}\right)^{*} \otimes \mathscr{F}\right)\right)
$$

can be considered as an odd self-adjoint element in

$$
\Gamma\left(\widetilde{B}, \operatorname{End}^{-}\left(\widetilde{\pi}_{*}^{\text {spin }}\left(S\left(T^{V} \widetilde{X}\right)^{*} \otimes \mathscr{F}\right)\right)\right) .
$$


We do not assume $\mathrm{D}^{S \widehat{\otimes}\left(S^{*} \otimes \mathscr{F}\right)}+\widetilde{V}$ satisfies Assumption 1, Let $\mathcal{L} \rightarrow \widetilde{B}$ be a $\mathbb{Z}_{2}$-graded complex vector bundle satisfying the MF property with respect to $\mathrm{D}^{S \widehat{\otimes}\left(S^{*} \otimes \mathscr{F}\right)}+\widetilde{V}$. As in the proof of Proposition 1] write $L \rightarrow B$ for $i_{B, 1}^{*} \mathcal{L} \rightarrow B$, and note that $i_{B, 1}^{*} \mathcal{L} \cong i_{B, 0}^{*} \mathcal{L}$.

Note that

$$
S\left(T^{V} X\right) \widehat{\otimes} S\left(T^{V} X\right)^{*} \cong \Lambda\left(T^{V} X\right)^{*} \otimes \mathbb{C}
$$

as $\mathbb{Z}_{2}$-graded complex vector bundles. By suppressing the notation $\otimes \mathbb{C}$ in 3.3.5 we have

$$
\begin{aligned}
S\left(T^{V} X\right) \widehat{\otimes}\left(S\left(T^{V} X\right)^{*} \otimes \mathrm{F}\right) & \cong\left(S\left(T^{V} X\right) \widehat{\otimes} S\left(T^{V} X\right)^{*}\right) \otimes \mathrm{F} \\
& \cong \Lambda\left(T^{V} X\right)^{*} \otimes \mathrm{F}
\end{aligned}
$$

as $\mathbb{Z}_{2}$-graded complex vector bundles. By (2.3.2), 2.4.2) and (3.3.6) we have $\pi_{*}^{\Lambda} \mathrm{F} \cong \pi_{*}^{\mathrm{spin}}\left(S\left(T^{V} X\right)^{*} \otimes \mathrm{F}\right)$, and therefore

$$
\Gamma\left(B, \pi_{*}^{\Lambda} \mathrm{F}\right) \cong \Gamma\left(B, \pi_{*}^{\text {spin }}\left(S\left(T^{V} X\right)^{*} \otimes \mathrm{F}\right)\right) .
$$

Moreover, under the isomorphism (3.3.5) the Clifford multiplication on the Clifford module $S\left(T^{V} X\right) \rightarrow X$ corresponds to the Clifford multiplication on the Clifford module on $\Lambda\left(T^{V} X\right)^{*} \rightarrow X$ [4, Proposition 3.5], and

$$
\nabla^{S\left(T^{V} X\right) \widehat{\otimes} S\left(T^{V} X\right)^{*}}=\nabla^{\Lambda\left(T^{V} X\right)^{*}}
$$

as $\mathbb{Z}_{2}$-graded unitary connections, where $\nabla^{S\left(T^{V} X\right) \widehat{\otimes} S\left(T^{V} X\right)^{*}}$ is the $\mathbb{Z}_{2}$-graded tensor product of $\nabla^{S\left(T^{V} X\right)}$ and $\nabla^{S\left(T^{V} X\right)^{*}}$.

Recall that $\mathrm{D}_{1}^{S \widehat{\otimes}\left(S^{*} \otimes \mathrm{F}\right)}+V_{1}$ is defined in terms of, among other things, $\left(\mathrm{F}, \nabla_{1}^{\mathrm{F}, u}\right)$, where $\nabla_{1}^{\mathrm{F}, u}=k \nabla^{+, u}$. By 2.3.1), 2.4.2 and 3.3.7 we have

$$
\mathrm{D}_{1}^{\Lambda \otimes \mathrm{F}}=\mathrm{D}_{1}^{S \widehat{\otimes}\left(S^{*} \otimes \mathrm{F}\right)}
$$

Thus by $(2.3 .8)$ and $(3.3 .8)$ we have

$$
\mathrm{D}_{1}^{Z, \mathrm{dR}}=\mathrm{D}_{1}^{\Lambda \otimes \mathrm{F}}+V_{1}=\mathrm{D}_{1}^{S \widehat{\otimes}\left(S^{*} \otimes \mathrm{F}\right)}+V_{1} .
$$

Since the family of kernels of $D_{1}^{Z, d R}$ form a $\mathbb{Z}_{2}$-graded complex vector bundle, the same is true for $\mathrm{D}_{1}^{S \widehat{\otimes}\left(S^{*} \otimes \mathrm{F}\right)}+V_{1}$. Denoted by $\operatorname{ker}\left(\mathrm{D}_{1}^{S \widehat{\otimes}\left(S^{*} \otimes \mathrm{F}\right)}+V_{1}\right) \rightarrow B$ the resulting $\mathbb{Z}_{2}$-graded complex vector bundle. Similarly, $\mathrm{D}_{0}^{S \widehat{\otimes}\left(S^{*} \otimes \mathrm{F}\right)}+V_{0}$ is defined in terms of, among other things, $\left(\mathrm{F}, \nabla_{0}^{\mathrm{F}, u}\right)$, where $\nabla_{0}^{\mathrm{F}, u}=\widetilde{j}^{*} k \nabla^{-, u}$. By a similar argument the family of the kernels of $\mathrm{D}_{0}^{S \widehat{\otimes}\left(S^{*} \otimes \mathrm{F}\right)}+V_{0}$ form a $\mathbb{Z}_{2}$-graded complex vector bundle, denoted by $\operatorname{ker}\left(\mathrm{D}_{0}^{S \hat{\otimes}\left(S^{*} \otimes \mathrm{F}\right)}+V_{0}\right) \rightarrow B$.

Let $i \in\{0,1\}$. Since $\operatorname{ker}\left(\mathrm{D}_{i}^{S \widehat{\otimes}\left(S^{*} \otimes \mathrm{F}\right)}+V_{i}\right) \rightarrow B$ satisfies the MF property with respect to $\mathrm{D}_{i}^{S \widehat{\otimes}\left(S^{*} \otimes \mathrm{F}\right)}+V_{i}$, by 3.1 .15 there exist $\mathbb{Z}_{2}$-graded complex vector bundles $H \rightarrow B$ and $W_{i} \rightarrow B$ of the form $H^{+}=H^{-}$and $W_{i}^{+}=W_{i}^{-}$ such that

$$
\operatorname{ker}\left(\mathrm{D}_{0}^{S \widehat{\otimes}\left(S^{*} \otimes \mathrm{F}\right)}+V_{0}\right) \oplus W_{0} \cong L \oplus H \cong \operatorname{ker}\left(\mathrm{D}_{1}^{S \widehat{\otimes}\left(S^{*} \otimes \mathrm{F}\right)}+V_{1}\right) \oplus W_{1}
$$


as $\mathbb{Z}_{2}$-graded complex vector bundles. By $(2.3 .5)$ and $(3.3 .9)$ we have

$$
\operatorname{ker}\left(\mathrm{D}_{1}^{S \widehat{\otimes}\left(S^{*} \otimes \mathrm{F}\right)}+V_{1}\right) \cong H\left(Z,\left.\mathrm{~F}\right|_{Z}\right)=H\left(Z,\left.k F^{+}\right|_{Z}\right)
$$

as $\mathbb{Z}_{2}$-graded complex vector bundles, where the flat connection $\left.\left.\nabla^{H\left(Z, k F^{+}\right.}\right|_{Z}\right)$ on $H\left(Z,\left.k F^{+}\right|_{Z}\right) \rightarrow B$ in 3.3.11 is defined in terms of $\nabla_{1}^{\mathrm{F}}=k \nabla^{+}$. By a similar argument we have

$$
\operatorname{ker}\left(\mathrm{D}_{0}^{S \widehat{\otimes}\left(S^{*} \otimes \mathrm{F}\right)}+V_{0}\right) \cong H\left(Z,\left.\mathrm{~F}\right|_{Z}, \nabla_{0}^{\mathrm{F}}\right)=H\left(Z,\left.k F^{+}\right|_{Z}, \tilde{j}^{*} k \nabla^{-}\right)
$$

as $\mathbb{Z}_{2}$-graded complex vector bundles. In 3.3 .12 the notation for the cohomology bundle is to emphasize that it is defined by the corresponding flat connection. Since $\left(k F^{+}, \widetilde{j}^{*} k \nabla^{-}\right) \cong\left(k F^{-}, k \nabla^{-}\right)$as complex flat vector bundles by (3.3.4), 3.3.12) becomes

$$
\operatorname{ker}\left(\mathrm{D}_{0}^{S \widehat{\otimes}\left(S^{*} \otimes \mathrm{F}\right)}+V_{0}\right) \cong H\left(Z,\left.k F^{+}\right|_{Z}, \widetilde{j}^{*} k \nabla^{-}\right) \cong H\left(Z,\left.k F^{-}\right|_{Z}\right)
$$

as $\mathbb{Z}_{2}$-graded complex vector bundles. Since for any complex flat vector bundle $E \rightarrow X$ with flat connection $\nabla^{E}$ and any $k \in \mathbb{N}$,

$$
\left(H\left(Z,\left.k E\right|_{Z}\right), \nabla^{H\left(Z,\left.k E\right|_{Z}\right)}\right) \cong\left(k H\left(Z,\left.E\right|_{Z}\right), k \nabla^{H\left(Z,\left.E\right|_{Z}\right)}\right)
$$

as $\mathbb{Z}$-graded complex flat vector bundles, it follows from 3.3.11, 3.3.12 and (3.3.13) that $(3.3 .10)$ becomes

$$
k H\left(Z,\left.F^{-}\right|_{Z}\right) \oplus W_{0} \cong L \oplus H \cong k H\left(Z,\left.F^{+}\right|_{Z}\right) \oplus W_{1} .
$$

Consider the following bundle isomorphism of the even part of (3.3.14):

$$
k H^{\text {even }}\left(Z,\left.F^{-}\right|_{Z}\right) \oplus W_{0}^{+} \cong k H^{\text {even }}\left(Z,\left.F^{+}\right|_{Z}\right) \oplus W_{1}^{+} \text {. }
$$

Since $B$ is assumed to be compact, there exists a complex vector bundle $V \rightarrow B$ such that $W_{1}^{+} \oplus V \cong \mathbb{C}^{m}$ for some $m \in \mathbb{N}$. By direct summing $V \rightarrow B$ and $\ell$ copies of $\mathbb{C}^{m} \rightarrow B$ on both sides of (3.3.15), where $\ell \in \mathbb{N}$, it becomes

$$
k H^{\text {even }}\left(Z,\left.F^{-}\right|_{Z}\right) \oplus \widetilde{W_{0}^{+}} \cong k H^{\text {even }}\left(Z,\left.F^{+}\right|_{Z}\right) \oplus \mathbb{C}^{(\ell+1) m},
$$

where $\widetilde{W_{0}^{+}}:=W_{0}^{+} \oplus V \oplus \mathbb{C}^{\ell m}$. By choosing and fixing a sufficiently large $\ell$, we may assume that $2 \operatorname{rank}\left(\widetilde{W_{0}^{+}}\right) \geq \operatorname{dim}(B)$. Since $k H^{\text {even }}\left(Z,\left.F^{ \pm}\right|_{Z}\right) \cong$ $k \mathbb{C}^{n_{ \pm}^{\text {even }}} \cong \mathbb{C}^{k n_{ \pm}^{\text {even }}}$ by 3.3.3, 3.3 .16 becomes

$$
\mathbb{C}^{k n_{-}^{\text {even }}} \oplus \widetilde{W_{0}^{+}} \cong \mathbb{C}^{k n_{+}^{\text {even }}+(\ell+1) m} .
$$

Thus $k n_{-}^{\text {even }}+\operatorname{rank}\left(\widetilde{W_{0}^{+}}\right)=k n_{+}^{\text {even }}+(\ell+1) m$. Since $\mathbb{C}^{k n_{+}^{\text {even }}+(\ell+1) m} \cong$ $\mathbb{C}^{k n_{-}^{\text {even }}} \oplus \mathbb{C}^{\operatorname{rank}\left(\widetilde{W_{0}^{+}}\right)}$, it follows that

$$
\mathbb{C}^{k n_{-}^{\text {even }}} \oplus \widetilde{W_{0}^{+}} \cong \mathbb{C}^{k n_{-}^{\text {even }}} \oplus \mathbb{C}^{\operatorname{rank}\left(\widetilde{W_{0}^{+}}\right)} .
$$

By [21, Theorem 1.5 of Chapter 9] (see also Remark 1) we have $\widetilde{W_{0}^{+}} \cong$ $\mathbb{C}^{\operatorname{rank}\left(\widetilde{W_{0}^{+}}\right)}$. Since $W_{0}^{-}=W_{0}^{+}$and $W_{1}^{-}=W_{1}^{+}$, it follows that

$$
\widetilde{W_{0}^{-}}:=W_{0}^{-} \oplus V \oplus \mathbb{C}^{\ell m}=\widetilde{W_{0}^{+}} \cong \mathbb{C}^{\operatorname{rank}\left(\widetilde{W_{0}^{+}}\right)} .
$$


The above argument shows that there exist sufficiently large $N, M \in \mathbb{N}$ such that the following diagram commutes

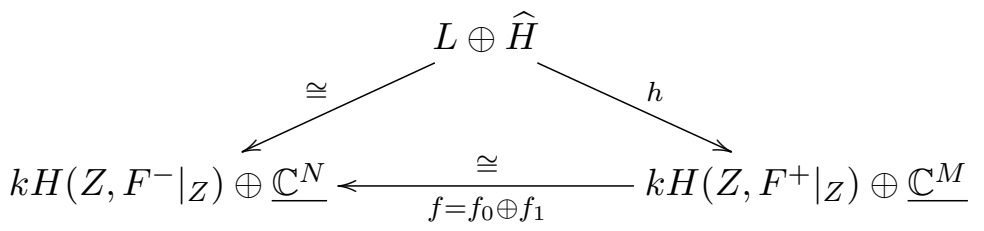

where $\widehat{H} \rightarrow B$ is the resulting $\mathbb{Z}_{2}$-graded complex vector bundle, and $f, h$ are the resulting smooth $\mathbb{Z}_{2}$-graded bundle isomorphisms.

Put $\mathbb{Z}_{2}$-graded trivial metrics $g^{N}$ and $g^{M}$ and $\mathbb{Z}_{2}$-graded trivial unitary connections $\underline{d^{N}}$ and $\underline{d^{M}}$ on $\underline{\mathbb{C}^{N}} \rightarrow B$ and $\underline{\mathbb{C}^{M}} \rightarrow B$ respectively. Since

$$
k g^{H^{\text {even }}\left(Z,\left.F^{+}\right|_{Z}\right)} \oplus g^{M}, \quad f_{0}^{*}\left(k g^{H^{\text {even }}\left(Z, F^{-} \mid Z\right)} \oplus g^{N}\right)
$$

are Hermitian metrics on $k H^{\text {even }}\left(Z,\left.F^{+}\right|_{Z}\right) \oplus \mathbb{C}^{M} \rightarrow B$, by 2.2.11 there exists a smooth isometric isomorphism

$$
\widetilde{f}_{0}: k H^{\text {even }}\left(Z,\left.F^{+}\right|_{Z}\right) \oplus \mathbb{C}^{M} \rightarrow k H^{\text {even }}\left(Z,\left.F^{-}\right|_{Z}\right) \oplus \mathbb{C}^{N} .
$$

Similarly, denote by

$$
\tilde{f}_{1}: k H^{\text {odd }}\left(Z,\left.F^{+}\right|_{Z}\right) \oplus \mathbb{C}^{M} \rightarrow k H^{\text {odd }}\left(Z,\left.F^{-}\right|_{Z}\right) \oplus \mathbb{C}^{N}
$$

the corresponding smooth isometric isomorphism. Then

$$
\widetilde{f}:=\widetilde{f}_{0} \oplus \tilde{f}_{1}: k H\left(Z,\left.F^{+}\right|_{Z}\right) \oplus \underline{\mathbb{C}^{M}} \rightarrow k H\left(Z,\left.F^{-}\right|_{Z}\right) \oplus \underline{\mathbb{C}^{N}}
$$

is a smooth $\mathbb{Z}_{2}$-graded isometric isomorphism, and $\tilde{f}^{*}\left(k \nabla^{H\left(Z, F^{-} \mid z\right), u} \oplus \underline{d^{N}}\right)$ is a $\mathbb{Z}_{2}$-graded unitary connection on $k H\left(Z,\left.F^{+}\right|_{Z}\right) \oplus \underline{\mathbb{C}^{M}} \rightarrow B$ with respect to $k g^{H\left(Z, F^{+} \mid Z\right)} \oplus \underline{g^{M}}$.

Note that $h^{*}\left(k \overline{\nabla^{H}}\left(Z,\left.F^{+}\right|_{z}\right), u \oplus \underline{d^{M}}\right)$ and $h^{*} \widetilde{f}^{*}\left(k \nabla^{H\left(Z, F^{-} \mid z\right), u} \oplus \underline{d^{N}}\right)$ are $\mathbb{Z}_{2^{-}}$ graded unitary connections on $L \oplus \widehat{H} \rightarrow B$ with respect to $h^{*}\left(k^{\left.\overline{H\left(Z, F^{+}\right.} \mid Z\right)} \oplus\right.$ $\left.g^{M}\right)$. Define a $\mathbb{Z}_{2}$-graded complex vector bundle $\mathcal{H} \rightarrow \widetilde{B}$ by $\mathcal{H}=p_{B}^{*} \widehat{H}$, and a unitary connection $\nabla^{\mathcal{L} \oplus \mathcal{H}}$ on $\mathcal{L} \oplus \mathcal{H} \rightarrow \widetilde{B}$ by 2.1.6 such that

$$
\begin{aligned}
& i_{B, 1}^{*} \nabla^{\mathcal{L} \oplus \mathcal{H}}=h^{*}\left(k \nabla^{H\left(Z,\left.F^{+}\right|_{Z}\right), u} \oplus \underline{d^{M}}\right), \\
& i_{B, 0}^{*} \nabla^{\mathcal{L} \oplus \mathcal{H}}=h^{*} \widetilde{f}^{*}\left(k \nabla^{H\left(Z, F^{-} \mid Z\right), u} \oplus \underline{d^{N}}\right) .
\end{aligned}
$$

The term $\widehat{A}\left(\nabla^{T^{V} \tilde{X}}\right) \wedge \operatorname{ch}\left(\nabla^{\mathscr{E}}\right)$ in 3.1 .19 becomes

$$
\widehat{A}\left(\nabla^{T^{V} \widetilde{X}}\right) \wedge \operatorname{ch}\left(\nabla^{S\left(T^{V} \widetilde{X}\right)^{*} \otimes \mathscr{F}, u}\right),
$$


where $\nabla^{S\left(T^{V} \tilde{X}\right)^{*} \otimes \mathscr{F}, u}$ is the tensor product of $\nabla^{S\left(T^{V} \widetilde{X}\right)^{*}}$ and $\nabla^{\mathscr{F}, u}$. By 1 , (8.30)] (see also [7, (3.46)] and [23, Proposition 11.24 in p.328]) we have

$$
\begin{aligned}
& \widehat{A}\left(\nabla^{T^{V} \tilde{X}}\right) \wedge \operatorname{ch}\left(\nabla^{S\left(T^{V} \tilde{X}\right)^{*} \otimes \mathscr{F}, u}\right) \\
= & \widehat{A}\left(\nabla^{T^{V} \widetilde{X}}\right) \wedge \operatorname{ch}\left(\nabla^{S\left(T^{V} \widetilde{X}\right)^{*}}\right) \wedge \operatorname{ch}\left(\nabla^{\mathscr{F}, u}\right) \\
= & \widehat{A}\left(\nabla^{T^{V} \widetilde{X}}\right) \wedge\left(\operatorname{ch}\left(\nabla^{S\left(T^{V} \widetilde{X}\right)^{*},+}\right)-\operatorname{ch}\left(\nabla^{S\left(T^{V} \widetilde{X}\right)^{*},-}\right)\right) \wedge \operatorname{ch}\left(\nabla^{\mathscr{F}, u}\right) \\
= & \widehat{A}\left(\nabla^{T^{V} \tilde{X}}\right) \wedge \frac{e\left(\nabla^{T^{V} \tilde{X}}\right)}{\widehat{A}\left(\nabla^{T^{V} \tilde{X}}\right)} \wedge \operatorname{ch}\left(\nabla^{\mathscr{F}, u}\right) \\
= & e\left(\nabla^{T^{V} \tilde{X}}\right) \wedge \operatorname{ch}\left(\nabla^{\mathscr{F}, u}\right) .
\end{aligned}
$$

By (3.1.5) and 3.3.20) we have

$$
\begin{aligned}
& \widehat{\eta}^{S\left(T^{V} X\right)^{*} \otimes \mathrm{F}}\left(k g^{+}, k \nabla^{+, u}, T^{H} X, g^{T^{V} X}, \operatorname{ker}\left(\mathrm{D}_{1}^{S \widehat{\otimes} S^{*} \otimes \mathrm{F}}+V_{1}\right)\right) \\
& -\widehat{\eta}^{S\left(T^{V} X\right)^{*} \otimes \mathrm{F}}\left(k g^{+}, \widetilde{j}^{*} k \nabla^{-, u}, T^{H} X, g^{T^{V} X}, \operatorname{ker}\left(\mathrm{D}_{0}^{S \widehat{\otimes} S^{*} \otimes \mathrm{F}}+V_{0}\right)\right) \\
= & \int_{X / B} e\left(\nabla^{T^{V} X}\right) \wedge \mathrm{CS}\left(\widetilde{j}^{*} k \nabla^{-, u}, k \nabla^{+, u}\right) \\
& -\mathrm{CS}\left(h^{*} \widetilde{f}^{*}\left(k \nabla^{H\left(Z, F^{-} \mid z\right), u} \oplus \underline{d^{N}}\right), h^{*}\left(k \nabla^{H\left(Z,\left.F^{+}\right|_{Z}\right), u} \oplus \underline{d^{M}}\right)\right)
\end{aligned}
$$

in $\frac{\Omega^{\text {odd }}(B)}{\operatorname{Im}(d)}$. Since $h$ covers the identity $\operatorname{map} \mathrm{id}_{B}$, it follows from 2.2 .10 that

$$
\begin{aligned}
& \operatorname{CS}\left(h^{*}\left(\widetilde{f}^{*} k \nabla^{H\left(Z,\left.F^{-}\right|_{Z}\right), u} \oplus \underline{d^{N}}\right), h^{*}\left(k \nabla^{H\left(Z,\left.F^{+}\right|_{Z}\right), u} \oplus \underline{d^{M}}\right)\right) \\
= & \operatorname{CS}\left(\widetilde{f}^{*}\left(k \nabla^{H\left(Z,\left.F^{-}\right|_{Z}\right), u} \oplus \underline{d^{N}}\right), k \nabla^{H\left(Z,\left.F^{+}\right|_{Z}\right), u} \oplus \underline{d^{M}}\right)
\end{aligned}
$$

in $\frac{\Omega^{\text {odd }}(B)}{\operatorname{Im}(d)}$. On the other hand, by 3.1 .35 we have

$$
\begin{aligned}
& \widehat{\eta}^{S\left(T^{V} X\right)^{*} \otimes \mathrm{F}}\left(k g^{+}, k \nabla^{+, u}, T^{H} X, g^{T^{V} X}, \operatorname{ker}\left(\mathrm{D}_{1}^{S \widehat{\otimes} S^{*} \otimes \mathrm{F}}+V_{1}\right)\right) \\
= & \widetilde{\eta}^{S\left(T^{V} X\right)^{*} \otimes \mathrm{F}}\left(k g^{+}, k \nabla^{+, u}, T^{H} X, g^{T^{V} X}\right)
\end{aligned}
$$

in $\frac{\Omega^{\text {odd }}(B)}{\operatorname{Im}(d)}$. By 3.3.7 and 3.3 .6 we have

$$
\begin{aligned}
& \nabla^{\Lambda\left(T^{V} X\right)^{*}} \otimes \operatorname{id}_{\Gamma(X, \mathrm{~F})}+\mathrm{id}_{\Gamma\left(X, \Lambda\left(T^{V} X\right)^{*}\right)} \otimes \nabla_{1}^{\mathrm{F}, u} \\
= & \nabla^{S\left(T^{V} X\right) \widehat{\otimes} S\left(T^{V} X\right)^{*}} \otimes \operatorname{id}_{\Gamma(X, \mathrm{~F})}+\mathrm{id}_{\Gamma\left(X, S\left(T^{V} X\right) \widehat{\otimes} S\left(T^{V} X\right)^{*}\right)} \otimes \nabla_{1}^{\mathrm{F}, u} \\
= & \nabla^{S\left(T^{V} X\right)} \otimes \mathrm{id}_{\Gamma\left(X, S\left(T^{V} X\right)^{*} \otimes \mathrm{F}\right)}+\mathrm{id}_{\Gamma\left(X, S\left(T^{V} X\right)\right)} \otimes \nabla^{S\left(T^{V} X\right)^{*} \otimes \mathrm{F}, u},
\end{aligned}
$$

where $\nabla^{S\left(T^{V} X\right)^{*} \otimes \mathrm{F}, u}$ is the tensor product connection of $\nabla^{S\left(T^{V} X\right)^{*}}$ and $\nabla_{1}^{\mathrm{F}, u}=$ $k \nabla^{+, u}$. It follows from 2.3.4 , 2.4.3 and above that

$$
\nabla^{\pi_{*}^{\Lambda} \mathrm{F}, u}=\nabla_{*}^{\pi_{*}^{\mathrm{spin}}}\left(S\left(T^{V} X\right)^{*} \otimes \mathrm{F}\right), u .
$$


By 3.3.8) and 3.3.23 we see that $\mathbb{B}^{S\left(T^{V} X\right)^{*} \otimes \mathrm{F}}=\mathbb{B}^{\mathrm{dR}}$. By 2.3.12 we have

$$
\widetilde{\eta}^{S\left(T^{V} X\right)^{*} \otimes \mathrm{F}}\left(k g^{+}, k \nabla^{+, u}, T^{H} X, g^{T^{V} X}\right)=\widetilde{\eta}^{\mathrm{dR}}=0 .
$$

A similar argument shows that

$$
\begin{aligned}
& \widehat{\eta}^{S\left(T^{V} X\right)^{*} \otimes \mathrm{F}}\left(k g^{+}, \widetilde{j}^{*} k \nabla^{-, u}, T^{H} X, g^{T^{V} X}, \operatorname{ker}\left(\mathrm{D}_{0}^{S \widehat{\otimes} S^{*} \otimes \mathrm{F}}+V_{0}\right)\right) \\
& =\widetilde{\eta}^{S\left(T^{V} X\right)^{*} \otimes \mathrm{F}}\left(k g^{+}, \widetilde{j}^{*} k \nabla^{-, u}, T^{H} X, g^{T^{V} X}\right)=\widetilde{\eta}^{\mathrm{dR}}=0 .
\end{aligned}
$$

By (3.3.22), (3.3.21) becomes

$$
\operatorname{CS}\left(\widetilde{f}^{*}\left(k \nabla^{H\left(Z,\left.F^{-}\right|_{Z}\right), u} \oplus \underline{d^{N}}\right), k \nabla^{H\left(Z, F^{+} \mid Z\right), u} \oplus \underline{d^{M}}\right)=\int_{X / B} e\left(\nabla^{T^{V} X}\right) \wedge \operatorname{CS}\left(\widetilde{j}^{*} k \nabla^{-, u}, k \nabla^{+, u}\right)
$$

in $\frac{\Omega^{\text {odd }}(B)}{\operatorname{Im}(d)}$. Since all the above computations are local, it follows that 3.3.24 holds without assuming the fibers $Z$ are oriented and spin. Thus (3.3.1) holds.

The following corollary is a $\mathbb{Z}_{2}$-graded version of $(1.0 .2$ for $\operatorname{dim}(Z)$ even.

Corollary 3. Let $\pi: X \rightarrow B$ be a submersion with closed fibers $Z$ with $\operatorname{dim}(Z)$ even, and $F \rightarrow X$ a $\mathbb{Z}_{2}$-graded complex flat vector bundle of virtual rank zero with $\mathbb{Z}_{2}$-graded flat connection $\nabla^{F}=\nabla^{+} \oplus \nabla^{-}$. By putting a $\mathbb{Z}_{2^{-}}$ graded Hermitian metric $g^{F}$ on $F \rightarrow X$ and the induced $\mathbb{Z}$-graded Hermitian metric $g^{H\left(Z,\left.F\right|_{Z}\right)}$ on $H\left(Z,\left.F\right|_{Z}\right) \rightarrow B$ we have

$$
\operatorname{Re}\left(\operatorname{CCS}\left(H\left(Z,\left.F\right|_{Z}\right), \nabla^{H\left(Z,\left.F\right|_{Z}\right)}\right)\right)=\int_{X / B} e\left(T^{V} X\right) \cup \operatorname{Re}\left(\operatorname{CCS}\left(F, \nabla^{F}\right)\right)
$$

in $H^{\text {odd }}(B ; \mathbb{R} / \mathbb{Q})$.

In the following proof we adopt the notations in the proof of Theorem 1 .

Proof. Let $F \rightarrow X$ be a $\mathbb{Z}_{2}$-graded complex flat vector bundle of virtual rank zero with $\mathbb{Z}_{2}$-graded flat connection $\nabla^{F}=\nabla^{+} \oplus \nabla^{-}$. As in the proof of Theorem 1 let $k \in \mathbb{N}$ be the least common multiple of the integers in (3.3.2.

Since the $\mathbb{Z}_{2}$-grading of $H\left(Z,\left.F\right|_{Z}\right) \rightarrow B$ is given by

$$
\begin{aligned}
& H\left(Z,\left.F\right|_{Z}\right)^{+}=H^{\text {even }}\left(Z,\left.F^{+}\right|_{Z}\right) \oplus H^{\text {odd }}\left(Z,\left.F^{-}\right|_{Z}\right), \\
& H\left(Z,\left.F\right|_{Z}\right)^{-}=H^{\text {even }}\left(Z,\left.F^{-}\right|_{Z}\right) \oplus H^{\text {odd }}\left(Z,\left.F^{+}\right|_{Z}\right),
\end{aligned}
$$

it follows that

$$
\begin{aligned}
& \operatorname{rank}\left(H\left(Z,\left.F\right|_{Z}\right)^{+}\right)-\operatorname{rank}\left(H\left(Z,\left.F\right|_{Z}\right)^{-}\right) \\
= & \operatorname{rank}\left(H^{\text {even }}\left(Z,\left.F^{+}\right|_{Z}\right)\right)+\operatorname{rank}\left(H^{\text {odd }}\left(Z,\left.F^{-}\right|_{Z}\right)\right)-\operatorname{rank}\left(H^{\text {even }}\left(Z,\left.F^{-}\right|_{Z}\right)\right) \\
& \quad-\operatorname{rank}\left(H^{\text {odd }}\left(Z,\left.F^{+}\right|_{Z}\right)\right) \\
= & \operatorname{rank}\left(H\left(Z,\left.F^{+}\right|_{Z}\right)\right)-\operatorname{rank}\left(H\left(Z,\left.F^{-}\right|_{Z}\right)\right) \\
= & \chi(Z) \operatorname{rank}\left(F^{+}\right)-\chi(Z) \operatorname{rank}\left(F^{-}\right)=0 .
\end{aligned}
$$


Thus by Lemma 3 we have

$$
\begin{aligned}
& \operatorname{Re}\left(\operatorname{CCS}\left(H\left(Z,\left.F\right|_{Z}\right), \nabla^{H\left(Z,\left.F\right|_{Z}\right)}\right)\right) \\
= & \operatorname{Re}\left(\operatorname{CCS}\left(H\left(Z,\left.F\right|_{Z}\right)^{+}, \nabla^{H\left(Z,\left.F\right|_{Z}\right)^{+}}\right)\right)-\operatorname{Re}\left(\operatorname{CCS}\left(H\left(Z,\left.F\right|_{Z}\right)^{-}, \nabla^{H\left(Z,\left.F\right|_{Z}\right)^{-}}\right)\right) \\
= & \operatorname{Re}\left(\operatorname{CCS}\left(H^{\text {even }}\left(Z,\left.F^{+}\right|_{Z}\right), \nabla^{H^{\text {even }}\left(Z,\left.F^{+}\right|_{Z}\right)}\right)\right)+\operatorname{Re}\left(\operatorname{CCS}\left(H^{\text {odd }}\left(Z,\left.F^{-}\right|_{Z}\right), \nabla^{H^{\text {odd }}\left(Z,\left.F^{-}\right|_{Z}\right)}\right)\right) \\
& -\operatorname{Re}\left(\operatorname{CCS}\left(H^{\text {even }}\left(Z,\left.F^{-}\right|_{Z}\right), \nabla^{H^{\text {even }}\left(Z,\left.F^{-}\right|_{Z}\right)}\right)\right)-\operatorname{Re}\left(\operatorname{CCS}\left(H^{\text {odd }}\left(Z,\left.F^{+}\right|_{Z}\right), \nabla^{H^{\text {odd }}\left(Z,\left.F^{+}\right|_{Z}\right)}\right)\right) .
\end{aligned}
$$

Recall from (3.3.3) that the choice of $k$ guarantees the existence of the smooth bundle isomorphisms $j: k F^{+} \rightarrow k F^{-}$and $h_{ \pm}^{\bullet}: k H^{\bullet}\left(Z, F^{ \pm}\right) \rightarrow$ $\mathbb{C}^{k n_{ \pm}^{\bullet}}$. Put a $\mathbb{Z}_{2}$-graded Hermitian metric $g^{F}$ on $F \rightarrow X$, and put the induced $\mathbb{Z}$-graded Hermitian metric $\left.\left.g^{H^{\bullet}\left(Z, F^{ \pm}\right.}\right|_{Z}\right)$ on $H^{\bullet}\left(Z,\left.F^{ \pm}\right|_{Z}\right) \rightarrow B$ as in Theorem 1 ,

By 2.1.14, the left-hand side of (3.3.1) can be written as

$$
\begin{aligned}
& \operatorname{CS}\left(\widetilde{f}^{*}\left(k \nabla^{H\left(Z, F^{-} \mid z\right), u} \oplus \underline{d^{N}}\right), k \nabla^{H\left(Z, F^{+} \mid Z\right), u} \oplus \underline{d^{M}}\right) \\
= & \operatorname{CS}\left(\widetilde{f}_{0}^{*}\left(k \nabla^{H^{\text {even }}\left(Z, F^{-} \mid z\right), u} \oplus d^{N}\right), k \nabla^{H^{\text {even }}\left(Z, F^{+} \mid Z\right), u} \oplus d^{M}\right) \\
& -\operatorname{CS}\left(\widetilde{f}_{1}^{*}\left(k \nabla^{H^{\text {odd }}\left(Z,\left.F^{-}\right|_{Z}\right), u} \oplus d^{N}\right), k \nabla^{H^{\text {odd }}\left(Z,\left.F^{+}\right|_{Z}\right), u} \oplus d^{M}\right)
\end{aligned}
$$

in $\frac{\Omega^{\text {odd }}(B)}{\operatorname{Im}(d)}$. Recall from 3.3 .3 and 3.3 .18 that we have the following diagram of smooth bundle isomorphisms

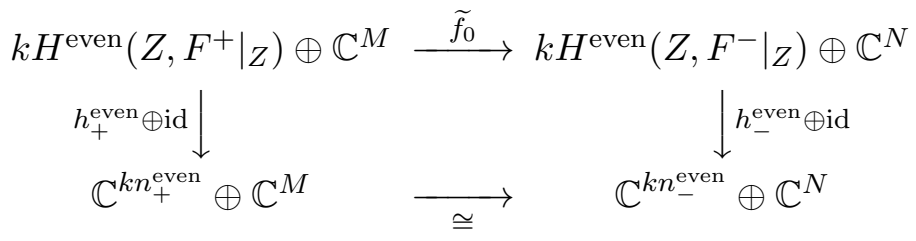

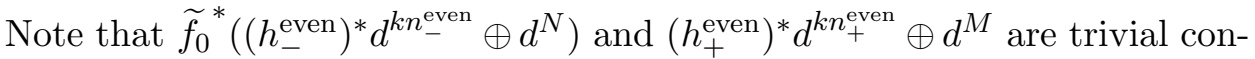
nections on $k H^{\text {even }}\left(Z,\left.F^{+}\right|_{Z}\right) \oplus \mathbb{C}^{M} \rightarrow B$. By 2.1.8, 2.1.9, 2.1.10, Remark 2 , and the fact that $\widetilde{f}_{0}$ covers the identity map id ${ }_{B}$ we have

$$
\begin{aligned}
& \operatorname{CS}\left(\widetilde{f}_{0}^{*}\left(k \nabla^{H^{\text {even }}\left(Z, F^{-} \mid Z\right), u} \oplus d^{N}\right), k \nabla^{H^{\text {even }}\left(Z,\left.F^{+}\right|_{Z}\right), u} \oplus d^{M}\right) \\
& =\operatorname{CS}\left(\widetilde{f}_{0}{ }^{*}\left(k \nabla^{H^{\text {even }}\left(Z, F^{-} \mid z\right), u} \oplus d^{N}\right),{\widetilde{f_{0}}}^{*}\left(\left(h_{-}^{\text {even }}\right)^{*} d^{k n_{-}^{\text {even }}} \oplus d^{N}\right)\right)
\end{aligned}
$$

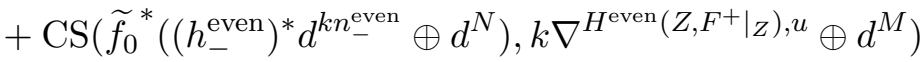

$$
\begin{aligned}
& =\operatorname{CS}\left(k \nabla^{H^{\text {even }}\left(Z,\left.F^{-}\right|_{Z}\right), u} \oplus d^{N},\left(h_{-}^{\text {even }}\right)^{*} d^{k n_{-}^{\text {even }}} \oplus d^{N}\right)
\end{aligned}
$$

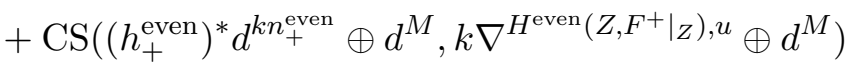

$$
\begin{aligned}
& =-\operatorname{CS}\left(\left(h_{-}^{\text {even }}\right)^{*} d^{k n_{-}^{\text {even }}}, k \nabla^{H^{\text {even }}\left(Z, F^{-} \mid z\right), u}\right)+\operatorname{CS}\left(\left(h_{+}^{\text {even }}\right)^{*} d^{k n_{+}^{\text {even }}}, k \nabla^{H^{\text {even }}\left(Z, F^{+} \mid z\right), u}\right)
\end{aligned}
$$


in $\frac{\Omega^{\text {odd }}(B)}{\Omega_{\mathbb{Q}}^{\text {odd }}(B)}$. By a similar argument we have

$$
\begin{aligned}
& -\operatorname{CS}\left(\widetilde{f}_{1}^{*}\left(k \nabla^{H^{\text {odd }}\left(Z, F^{-} \mid z\right), u} \oplus d^{N}\right), k \nabla^{H^{\text {odd }}\left(Z, F^{+} \mid z\right), u} \oplus d^{M}\right)
\end{aligned}
$$

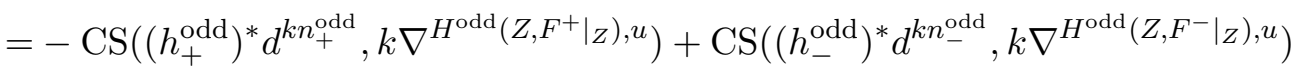

in $\frac{\Omega^{\text {odd }}(B)}{\Omega_{\mathbb{Q}}^{\text {odd }}(B)}$. By 3.3.28 and 3.3.29, 3.3.27 becomes

$$
\begin{aligned}
& \operatorname{CS}\left(\tilde{f}^{*}\left(k \nabla^{H\left(Z,\left.F^{-}\right|_{Z}\right), u} \oplus \underline{d^{N}}\right), k \nabla^{H\left(Z,\left.F^{+}\right|_{Z}\right), u} \oplus \underline{d^{M}}\right) \\
= & \operatorname{CS}\left(\left(h_{+}^{\text {even }}\right)^{*} d^{k n_{+}^{\text {even }}}, k \nabla^{H^{\text {even }}\left(Z,\left.F^{+}\right|_{Z}\right), u}\right)-\operatorname{CS}\left(\left(h_{-}^{\text {even }}\right)^{*} d^{k n_{-}^{\text {even }}}, k \nabla^{H^{\text {even }}\left(Z,\left.F^{-}\right|_{Z}\right), u}\right) \\
& -\operatorname{CS}\left(\left(h_{+}^{\text {odd }}\right)^{*} d^{k n_{+}^{\text {odd }}}, k \nabla^{H^{\text {odd }}\left(Z,\left.F^{+}\right|_{Z}\right), u}+\operatorname{CS}\left(\left(h_{-}^{\text {odd }}\right)^{*} d^{k n_{-}^{\text {odd }}}, k \nabla^{H^{\text {odd }}\left(Z,\left.F^{-}\right|_{Z}\right), u}\right)\right.
\end{aligned}
$$

in $\frac{\Omega^{\text {odd }}(B)}{\Omega_{\mathbb{Q}}^{\text {odd }}(B)}$. Therefore 3.3.1 becomes

$$
\begin{aligned}
& \operatorname{CS}\left(\left(h_{+}^{\text {even }}\right)^{*} d^{k n_{+}^{\text {even }}}, k \nabla^{H^{\text {even }}\left(Z,\left.F^{+}\right|_{Z}\right), u}\right)-\operatorname{CS}\left(\left(h_{-}^{\text {even }}\right)^{*} d^{\left.k n_{-}^{\text {even }},\left.k \nabla^{H^{\text {even }}\left(Z, F^{-}\right.}\right|_{Z}\right), u}\right) \\
& -\operatorname{CS}\left(\left(h_{+}^{\text {odd }}\right)^{*} d^{k n_{+}^{\text {odd }}}, k \nabla^{H^{\text {odd }}\left(Z,\left.F^{+}\right|_{Z}\right), u}+\operatorname{CS}\left(\left(h_{-}^{\text {odd }}\right)^{*} d^{k n_{-}^{\text {odd }}}, k \nabla^{H^{\text {odd }}\left(Z,\left.F^{-}\right|_{Z}\right), u}\right)\right. \\
= & \int_{X / B} e\left(\nabla^{T^{V} X}\right) \wedge \operatorname{CS}\left(\widetilde{j}^{*} k \nabla^{-, u}, k \nabla^{+, u}\right)
\end{aligned}
$$

in $\frac{\Omega^{\text {odd }}(B)}{\Omega_{\mathbb{Q}}^{\text {odd }}(B)}$. Since $\left(h_{ \pm}^{\bullet}\right)^{*} d^{k n_{ \pm}^{\bullet}}$ is a trivial connection on $k H^{\bullet}\left(Z,\left.F^{ \pm}\right|_{Z}\right) \rightarrow B$, it follows from (3.3.26) that the left-hand side of (3.3.31) is a differential form respective of left-hand side of (3.3.25), and the right-hand side of (3.3.31) is a differential form respective of the right-hand side of (3.3.25). Thus (3.3.31) implies that (3.3.25) holds.

Let $F \rightarrow X$ be a $\mathbb{Z}_{2}$-graded complex flat vector bundle with $\mathbb{Z}_{2}$-graded flat connection $\nabla^{F}$. By (3.3.26) and Lemma 2 we have

$$
\begin{aligned}
\operatorname{Re}\left(\operatorname{CCS}\left(H\left(Z,\left.F\right|_{Z}\right), \nabla^{H\left(Z,\left.F\right|_{Z}\right)}\right)\right)= & \sum_{k=0}(-1)^{k} \operatorname{Re}\left(\operatorname{CCS}\left(H^{k}\left(Z,\left.F^{+}\right|_{Z}\right), \nabla^{H^{k}\left(Z,\left.F^{+}\right|_{Z}\right)}\right)\right) \\
& -\sum_{k=0}(-1)^{k} \operatorname{Re}\left(\operatorname{CCS}\left(H^{k}\left(Z,\left.F^{-}\right|_{Z}\right), \nabla^{H^{k}\left(Z,\left.F^{-}\right|_{Z}\right)}\right)\right) .
\end{aligned}
$$

We now deduce 1.0 .2 for $\operatorname{dim}(Z)$ even.

Corollary 4. Let $\pi: X \rightarrow B$ be a submersion with closed fibers $Z$ with $\operatorname{dim}(Z)$ even, and $F \rightarrow X$ a complex flat vector bundle with flat connection 
$\nabla^{F}$. By putting a Hermitian metric $g^{F}$ on $F \rightarrow X$ and the induced $\mathbb{Z}$-graded Hermitian metric $g^{H\left(Z,\left.F\right|_{Z}\right)}$ on $H\left(Z,\left.F\right|_{Z}\right) \rightarrow B$ we have

$$
\sum_{k=0}^{n}(-1)^{k} \operatorname{Re}\left(\operatorname{CCS}\left(H^{k}\left(Z,\left.F\right|_{Z}\right), \nabla^{H^{k}\left(Z,\left.F\right|_{Z}\right)}\right)\right)=\int_{X / B} e\left(T^{V} X\right) \cup \operatorname{Re}\left(\operatorname{CCS}\left(F, \nabla^{F}\right)\right)
$$

in $H^{\text {odd }}(B ; \mathbb{R} / \mathbb{Q})$.

Proof. Let $F \rightarrow X$ be a complex flat vector bundle with flat connection $\nabla^{F}$. Write $\ell=\operatorname{rank}(F)$. Put a Hermitian metric $g^{F}$ on $F \rightarrow X$ and a trivial metric $g^{\ell}$ on $\mathbb{C}^{\ell} \rightarrow X$. Choose and fix a trivial connection $d^{\ell}$ on $\mathbb{C}^{\ell} \rightarrow X$. Define a $\mathbb{Z}_{2}$-graded complex vector bundle $\mathbb{F} \rightarrow X$ by $\mathbb{F}^{+}=F$ and $\mathbb{F}^{-}=\mathbb{C}^{\ell}$, and a $\mathbb{Z}_{2}$-graded connection $\nabla^{\mathbb{F}}=\nabla^{+} \oplus \nabla^{-}$on $\mathbb{F} \rightarrow X$, where $\nabla^{+}=\nabla^{F}$ and $\nabla^{-}=d^{\ell}$. Then $\mathbb{F} \rightarrow X$ is a $\mathbb{Z}_{2}$-graded complex flat vector bundle of virtual rank zero with $\mathbb{Z}_{2}$-graded flat connection $\nabla^{\mathbb{F}}$, and is equipped with a $\mathbb{Z}_{2}$-graded Hermitian metric $g^{F} \oplus g^{\ell}$. By (3.2.10 and Remark 2 we have

$$
\operatorname{CCS}\left(\mathbb{F}, \nabla^{\mathbb{F}}\right)=\operatorname{CCS}\left(F, \nabla^{F}\right)-\operatorname{CCS}\left(\mathbb{C}^{\ell}, d^{\ell}\right)=\operatorname{CCS}\left(F, \nabla^{F}\right)
$$

in $H^{\text {odd }}(B ; \mathbb{C} / \mathbb{Q})$. Thus

$$
\operatorname{Re}\left(\operatorname{CCS}\left(\mathbb{F}, \nabla^{\mathbb{F}}\right)\right)=\operatorname{Re}\left(\operatorname{CCS}\left(F, \nabla^{F}\right)\right)
$$

in $H^{\text {odd }}(B ; \mathbb{R} / \mathbb{Q})$. Denote by $g^{H\left(Z,\left.\mathbb{F}\right|_{Z}\right)}$ the induced $\mathbb{Z}$-graded Hermitian metric on the $\mathbb{Z}$-graded complex flat vector bundle $H\left(Z,\left.\mathbb{F}\right|_{Z}\right) \rightarrow B$ with $\mathbb{Z}$-graded flat connection $\nabla^{H\left(Z,\left.\mathbb{F}\right|_{Z}\right)}$. By applying Corollary 3 to $\left(\mathbb{F}, \nabla^{\mathbb{F}}\right)$, (3.3.25 and (3.3.34) imply

$$
\operatorname{Re}\left(\operatorname{CCS}\left(H\left(Z,\left.\mathbb{F}\right|_{Z}\right), \nabla^{H\left(Z,\left.\mathbb{F}\right|_{Z}\right)}\right)\right)=\int_{X / B} e\left(T^{V} X\right) \cup \operatorname{Re}\left(\operatorname{CCS}\left(F, \nabla^{F}\right)\right) .
$$

By (3.3.32), the left-hand side of (3.3.35) becomes

$$
\begin{aligned}
& \operatorname{Re}\left(\operatorname{CCS}\left(H\left(Z,\left.\mathbb{F}\right|_{Z}\right), \nabla^{H\left(Z,\left.\mathbb{F}\right|_{Z}\right)}\right)\right) \\
& =\sum_{k=0}(-1)^{k} \operatorname{Re}\left(\operatorname{CCS}\left(H^{k}\left(Z,\left.F\right|_{Z}\right), \nabla^{H^{k}\left(Z,\left.F\right|_{Z}\right)}\right)\right) \\
& \quad-\sum_{k=0}(-1)^{k} \operatorname{Re}\left(\operatorname{CCS}\left(H^{k}\left(Z,\left.\mathbb{C}^{\ell}\right|_{Z}\right), \nabla^{H^{k}\left(Z,\left.\mathbb{C}^{\ell}\right|_{Z}\right)}\right)\right) \\
& =\sum_{k=0}(-1)^{k} \operatorname{Re}\left(\operatorname{CCS}\left(H^{k}\left(Z,\left.F\right|_{Z}\right), \nabla^{H^{k}\left(Z,\left.F\right|_{Z}\right)}\right)\right) \\
& \quad-\operatorname{rank}(F) \sum_{k=0}(-1)^{k} \operatorname{Re}\left(\operatorname{CCS}\left(H^{k}\left(Z,\left.\mathbb{C}\right|_{Z}\right), \nabla^{H^{k}\left(Z, \mathbb{C}_{Z}\right)}\right)\right) .
\end{aligned}
$$

As argued in [27, p.614], the second part of [7, Theorem 3.12] implies that

$$
\sum_{k=0}^{n}(-1)^{k} \operatorname{Re}\left(\operatorname{CCS}\left(H^{k}\left(Z,\left.\mathbb{C}\right|_{Z}\right), \nabla^{H^{k}\left(Z,\left.\mathbb{C}\right|_{Z}\right)}\right)\right)=0
$$


in $H^{\text {odd }}(B ; \mathbb{R} / \mathbb{Q})$. Thus 3.3 .36 becomes

$$
\operatorname{Re}\left(\operatorname{CCS}\left(H\left(Z,\left.\mathbb{F}\right|_{Z}\right), \nabla^{H\left(Z,\left.\mathbb{F}\right|_{Z}\right)}\right)\right)=\sum_{k=0}^{n}(-1)^{k} \operatorname{Re}\left(\operatorname{CCS}\left(H^{k}\left(Z,\left.F\right|_{Z}\right), \nabla^{H^{k}\left(Z,\left.F\right|_{Z}\right)}\right)\right)
$$

in $H^{\text {odd }}(B ; \mathbb{R} / \mathbb{Q})$. It follows from 3.3 .35 and 3.3 .37 that 3.3 .33 holds.

\section{REFERENCES}

1. Michael F. Atiyah and Raoul Bott, A Lefschetz fixed point formula for elliptic complexes. II. Applications, Ann. of Math. (2) 88 (1968), 451-491.

2. Michael F. Atiyah, Vijay K. Patodi, and Isadore M. Singer, Spectral asymmetry and Riemannian geometry. III, Math. Proc. Cambridge Philos. Soc. 79 (1976), no. 1, 71-99.

3. Michael F. Atiyah and Isadore M. Singer, The index of elliptic operators. IV, Ann. of Math. (2) 93 (1971), 119-138.

4. Nicole Berline, Ezra Getzler, and Michèle Vergne, Heat kernels and Dirac operators, Grundlehren Text Editions, Springer-Verlag, Berlin, 2004, Corrected reprint of the 1992 original.

5. Jean-Michel Bismut, The Atiyah-Singer index theorem for families of Dirac operators: two heat equation proofs, Invent. Math. 83 (1986), no. 1, 91-151.

6. L Local index theory, eta invariants and holomorphic torsion: a survey, Surveys in differential geometry, Vol. III (Cambridge, MA, 1996), Int. Press, Boston, MA, 1998, pp. 1-76.

7. _ Eta invariants, differential characters and flat vector bundles, Chinese Ann. Math. Ser. B 26 (2005), 15-44.

8. Jean-Michel Bismut and Jeff Cheeger, $\eta$-invariants and their adiabatic limits, J. Amer. Math. Soc. 2 (1989), 33-70.

9. Jean-Michel Bismut and Daniel S. Freed, The analysis of elliptic families. I. Metrics and connections on determinant bundles, Comm. Math. Phys. 106 (1986), no. 1, 159176.

10. holonomy theorem, Comm. Math. Phys. 107 (1986), no. 1, 103-163.

11. Jean-Michel Bismut and John Lott, Flat vector bundles, direct images and higher real analytic torsion, J. Amer. Math. Soc. 8 (1995), no. 2, 291-363.

12. Jean-Michel Bismut and Weiping Zhang, An extension of a theorem by Cheeger and Müller, Astérisque (1992), no. 205, 235, With an appendix by François Laudenbach.

13. Raoul Bott and Loring W. Tu, Differential forms in algebraic topology, Graduate Texts in Mathematics, vol. 82, Springer-Verlag, New York-Berlin, 1982.

14. Jochen Brüning and Xiaonan Ma, An anomaly formula for Ray-Singer metrics on manifolds with boundary, Geom. Funct. Anal. 16 (2006), no. 4, 767-837.

15. Jeff Cheeger and James Simons, Differential characters and geometric invariants, in Geometry and Topology (College Park, Md., 1983/84), Lecture Notes in Math. 1167 (1985), 50-80.

16. Xianzhe Dai, Adiabatic limits, nonmultiplicativity of signature, and Leray spectral sequence, J. Amer. Math. Soc. 4 (1991), 265-321.

17. Daniel S. Freed and John Lott, An index theorem in differential K-theory, Geom. Topol. 14 (2010), 903-966.

18. Alexander Gorokhovsky and John Lott, A Hilbert bundle description of differential K-theory, Adv. Math. 328 (2018), 661-712. 
19. Werner Greub, Stephen Halperin, and Ray Vanstone, Connections, curvature, and cohomology. Vol. I: De Rham cohomology of manifolds and vector bundles, Academic Press, New York-London, 1972, Pure and Applied Mathematics, Vol. 47.

20. Man-Ho Ho, The flat Grothendieck-Riemann-Roch theorem without adiabatic techniques, J. Geom. Phys. 107 (2016), 162-174.

21. Dale Husemoller, Fibre bundles, third ed., Graduate Texts in Mathematics, vol. 20, Springer-Verlag, New York, 1994.

22. Max Karoubi, K-theory, Classics in Mathematics, Springer-Verlag, Berlin, 2008, An introduction, Reprint of the 1978 edition, With a new postface by the author and a list of errata.

23. H. Blaine Lawson, Jr. and Marie-Louise Michelsohn, Spin geometry, Princeton Mathematical Series, vol. 38, Princeton University Press, Princeton, NJ, 1989.

24. Bo Liu, Functoriality of equivariant eta forms, J. Noncommut. Geom. 11 (2017), no. 1, $225-307$.

25. John Lott, $\mathbb{R} / \mathbb{Z}$ index theory, Comm. Anal. Geom. 2 (1994), 279-311.

26. Xiaonan Ma and George Marinescu, Holomorphic Morse inequalities and Bergman kernels, Progress in Mathematics, vol. 254, Birkhäuser Verlag, Basel, 2007.

27. Xiaonan Ma and Weiping Zhang, Eta-invariants, torsion forms and flat vector bundles, Math. Ann. 340 (2008), no. 3, 569-624.

28. Alexandr S. Miščenko and Anatoliǔ T. Fomenko, The index of elliptic operators over $C^{*}$-algebras, Izv. Akad. Nauk SSSR Ser. Mat. 43 (1979), no. 4, 831-859, 967.

29. James Simons and Dennis Sullivan, Structured vector bundles define differential $K$ theory, Quanta of maths, Clay Math. Proc., vol. 11, Amer. Math. Soc., Providence, RI, 2010, pp. 579-599.

30. Weiping Zhang, Sub-signature operators, $\eta$-invariants and a Riemann-Roch theorem for flat vector bundles, Chinese Ann. Math. Ser. B 25 (2004), no. 1, 7-36.

Alumni of Boston University, Hong Kong

E-mail address: homanho@bu.edu 